\title{
Dilemma at RF Energy Harvesting Relay: Downlink Energy Relaying or Uplink Information Transfer?
}

Deepak Mishra, Swades De and Dilip Krishnaswamy

The self-archived postprint version of this journal article is available at Linköping University Institutional Repository (DiVA):

http:// urn.kb.se/ resolve?urn=urn:nbn:se:liu:diva- 155748

N.B.: When citing this work, cite the original publication.

Mishra, D., De, S., Krishnaswamy, D., (2017), Dilemma at RF Energy Harvesting Relay: Downlink Energy Relaying or Uplink Information Transfer?, IEEE Transactions on Wireless Communications, 16(8), 4939-4955. https:/ / doi.org/ 10.1109/ TWC.2017.2704084

Original publication available at:

https:/ / doi.org/ 10.1109/TWC.2017.2704084

Copyright: Institute of Electrical and Electronics Engineers (IEEE)

http:// www.ieee.org/ index.html

(C)2017 IEEE. Personal use of this material is permitted. However, permission to reprint/republish this material for advertising or promotional purposes or for creating new collective works for resale or redistribution to servers or lists, or to reuse any copyrighted component of this work in other works must be obtained from the IEEE. 


\title{
Dilemma at RF Energy Harvesting Relay: Downlink Energy Relaying or Uplink Information Transfer?
}

\author{
Deepak Mishra, Swades De, and Dilip Krishnaswamy
}

\begin{abstract}
Performance of RF powered communication network is bottlenecked by short downlink energy transfer range and doubly-near-far problem faced in uplink information transfer to Hybrid Access Point (HAP). These problems can be resolved by cooperation of an RF energy harvesting node $\mathcal{R}$ present between HAP and RF energy harvesting information source $\mathcal{S}$. However, there lies a dilemma at $\mathcal{R}$ on whether to transfer its harvested energy to $\mathcal{S}$ or to act as an information relay for transferring its data to HAP in a two-hop fashion. This paper resolves this dilemma at $\mathcal{R}$ by providing insights on its optimal positions suited for either energy relaying (ER) or information relaying (IR). It also investigates the possibilities of integrated ER and IR along with the regions where neither ER nor IR will be useful. In this regard, while considering Rician fading channels and practical nonlinear RF energy harvesting model, the expression for mean harvested dc power at $\mathcal{S}$ via energy transfer from HAP and ER from $\mathcal{R}$ is first derived. The closedform outage probability expression is also derived for decodeand-forward relaying with maximal-ratio-combining at HAP over Rician channels. Using these expressions insights on optimal relaying mode is obtained along with global-optimal utilization of harvested energy at $\mathcal{R}$ for ER and IR to maximize the delaylimited RF-powered throughput. Numerical results validate the analysis and provide insights on the optimal relaying mode.

Index Terms-Integrated information and energy relaying, practical RF energy harvesting model, Rician fading, outage analysis, throughput maximization, generalized convexity
\end{abstract}

\section{INTRODUCTION}

Radio frequency (RF) energy transfer (ET) has drawn wide recent attention due to its capability of providing controlled energy replenishment of low-power wireless devices. Unlike the inductive and magnetic resonant coupling based nonradiative ET approaches, radiative RF-ET bestows benefits [2] like relaxed node-alignment requirements, beamforming capability, and possibility of transmitting both energy and information over the same signal. This has led to the emergence of two attractive solutions for powering next generation networks: (a) Wireless Powered Communication Network (WPCN) [3] and (b) Simultaneous Wireless Information and Power Transfer (SWIPT) [4]. In WPCN, uplink information transfer (IT) is powered by downlink ET from Hybrid Access Point (HAP), whereas in SWIPT both ET and IT occur in same direction.

Despite the merits of RF-ET, there are some bottlenecks in its widespread usage. Major challenges [5] include wireless

D. Mishra and S. De are with the Department of Electrical Engineering, Indian Institute of Technology Delhi, New Delhi, India (e-mail: \{deepak.mishra, swadesd\}@ee.iitd.ac.in). D. Krishnaswamy is with IBM India Research Laboratory, Bangalore, India (e-mail: dilikris@in.ibm.com).

This work was supported by the Department of Science and Technology (DST) under Grant SB/S3/EECE/0248/2014 and by the 2016-2017 IBM PhD Fellowship Awards program. A preliminary version [1] of this work has been accepted for presentation at the IEEE ICC 2017. propagation and energy dissipation losses, low energy sensitivity, low rectification efficiency at low input power, and doubly-near-far problem [6] in WPCN. Therefore, investigation of new paradigms is needed for efficient WPCN operation.

\section{A. Related Art}

Various aspects of cooperative relaying have got recent research attention [5]-[20] to overcome doubly-near-far problem [21] in WPCN and large difference between energy and information sensitivities $(-10 \mathrm{dBm}$ versus $-60 \mathrm{dBm})$ in SWIPT. The authors in [5]-[10], [16]-[20] considered energy harvesting relay where an energy constrained node uses the harvested energy for cooperation. Optimal RF harvesting energy relay placement was investigated in [5] for maximizing the received power in two-hop RF-ET, both with and without distributed beamforming. In [6], a nearby node to HAP was considered to act as energy harvesting information relay for the farther node. A harvest-then-cooperate protocol was proposed in [7], where the relay node close to HAP harvests energy during downlink ET from HAP and then uses this energy for uplink information relaying (IR). The authors in [19] investigated instantaneous and delay-constrained throughput maximization for RF-powered full-duplex MIMO relay system by designing receive and transmit beamformers while optimizing the time-splitting parameter. A three-node RF-powered relay system was studied in [20] to maximize the ergodic throughput by optimizing the mode switching rule and transmit power jointly under the data and energy causality constraints.

Different from [6], [7] which considered fixed relaying, the approach in [8] dynamically decides whether the nearby node should act like an information relay for far node or not. Further in [9], the roles as source, destination, or relay, for the nodes were dynamically decided. Optimal allocation of harvested energy at relay, due to SWIPT from multiple sources for forwarding data to their respective receivers, was considered in [10]. In [16] a greedy protocol was proposed for switching between energy harvesting and data relaying to minimize outage probability in amplify-and-forward (AF) energy harvesting relay network without direct link. In [17] this work was extended to distributed multi-relay selection with decode-and-forward (DF) two-hop IT. More recently, a relay selection scheme, incorporating channel conditions and battery status, was proposed in [18] to choose one among multiple AF energy harvesting relays for IT. Furthermore, energy cooperation and sharing strategies have been proposed in [11], [12] to overcome dynamics of ambient energy harvesting and enable perpetual operation. In another set of works [13]-[15], 


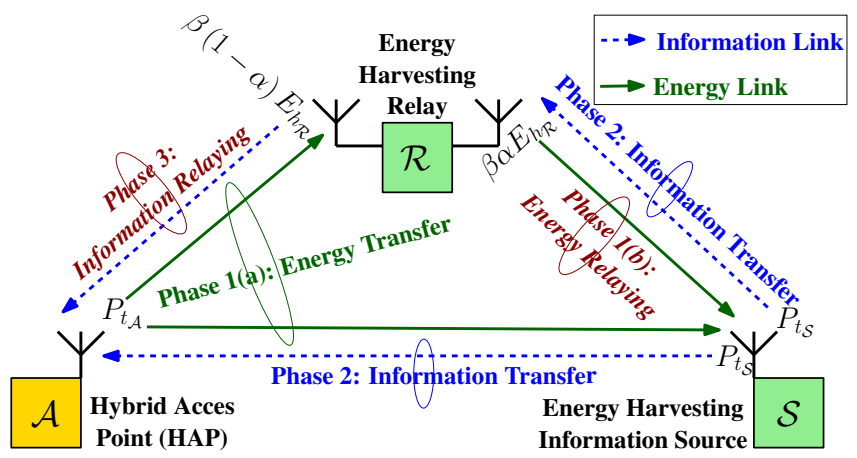

Fig. 1: Network topology for the RF-powered integrated 2-hop fullduplex energy relaying and half-duplex decode-and-forward IR.

relay-powered communications was considered, where energysufficient relay transfers energy to RF harvesting nodes.

\section{B. Motivation and Contributions}

In recent studies, harvested energy at relay is either used for energy relaying (ER) [5] or IR [6]-[10], [16]-[20]. Though [13]-[15] studied tradeoff in ET and IR efficiency assuming energy-rich relay, these works along with [11], [12] did not investigate RF harvesting relay assisted ER possibilities. This work fills this existing research gap. It studies optimal utilization of harvested energy at RF-energy harvesting $(\mathrm{EH})$ relay for ER and/or IR to enhance the performance of two-hop RFpowered delay-limited network with direct link availability. Key contributions of this work are six-fold: (1) As shown in Fig. 1, a novel system model is presented to investigate the performance of $R F$-powered integrated information and Energy Relaying $\left(i^{2} E R\right)$ in WPCN (Section II). (2) Mean harvested energy due to full-duplex ER over Rician fading channels with distributed beamforming is derived while considering practical RF harvesting model (Sections III). (3) Closed-form expressions for outage probability and normalized throughput are obtained for half-duplex DF-IR over Rician channels with Maximal Ratio Combining (MRC) (Section IV). (4) Analytical insights on optimal mode selection policy at RF harvesting relay are provided (Section V). (5) Global-optimal utilization of harvested energy at EH relay is obtained by efficiently solving the nonconvex delay-limited throughput maximization problem (Section VI). (6) Numerical results validate the analysis and give insights on optimal harvested energy utilization in ER and IR for varying relay positions (Section VII).

\section{Novelty and Scope}

To the best of our knowledge, this is the first work that considers $i^{2} E R$ in WPCN and resolves the "dilemma" at RF harvesting relay on whether to perform $E R, I R$, or $i^{2} E R$ by jointly optimizing cooperation in ET and IT. We also present novel analyses on full-duplex ER with distributed beamforming and half-duplex DF-IR with MRC over Rician channels.

Results presented in this paper demonstrate the importance of $i^{2} E R$, because in static node deployment scenarios where the relay position is fixed or the set of available routers are known, mode selection (ER or IR) becomes critical. This work providing insights on optimized mode of cooperation with RF harvesting relay (for energy, information, or both) can be extended to multi-node scenarios, allowing IR on one path and ER on other for greater end-to-end efficiency depending on the position of the relays. Energy beamforming [3] can also be applied over the proposed optimized cooperation for further enhancement of achievable gains. Though the widespread utility of the proposed system architecture is constrained by the low RF-ET range [2], [5], there are some practical applications that can benefit from this proposal. These include low power EH nodes in small cell networks, miniature RF-powered sensing devices for indoor applications, and EH nodes in Internet-ofThings. Furthermore, with the advancement in RF-EH circuits technology [22], [23], this limited end-to-end RF-ET range will be significantly increased due to improvement in both RF-to-dc rectification efficiency and receive energy sensitivity. Another attractive solution to improve the performance of the proposed RF-powered $i^{2} E R$ is by implementing the fullduplex IR. However this improvement comes at the expense of implementing loopback interference suppression with the help of sophisticated electronic schemes or spatial domain precoding techniques that require perfect channel estimation. So there is a need for low-cost energy-efficient full-duplex IR techniques for RF-powered relaying systems.

\section{SySTEM MODEL}

Here we present the $i^{2} E R$ system model that includes transmission protocol, network topology, and wireless channel, along with the energy consumption and RF harvesting models.

\section{A. Integrated Information and Energy Relaying in WPCN}

We consider RF-EH relay $\mathcal{R}$ assisted full-duplex two-hop downlink ET to RF-EH information source $\mathcal{S}$ and DF halfduplex uplink IT to HAP $\mathcal{A}$ (cf. Fig. 1). We assume that $\mathcal{A}$ and $\mathcal{S}$ are composed of single omnidirectional antennas, whereas $\mathcal{R}$ has two directional antennas; one pointing in the direction of $\mathcal{A}$ - essentially for efficient $\mathrm{EH}$ at $\mathcal{R}$ and effective IR from $\mathcal{R}$-to- $\mathcal{A}$, and other directed towards $\mathcal{S}$ for efficient downlink ER and improving the quality of $\mathcal{S}$-to- $\mathcal{R}$ uplink IT. Although half-duplex IR can be conducted using a single omnidirectional antenna at $\mathcal{R}$, two directional antennas are considered to minimize the dissipation losses in downlink ER and uplink IR. Also, this helps in implementing twohop full-duplex ER because RF energy signals do not contain any information to be lost during full-duplex operation. The role of full-duplex relaying in ER phase is to ensure that $\mathcal{R}$ can simultaneously harvest energy from $\mathcal{A}$ as well transfer energy to $\mathcal{S}$ in the $N_{s}$ th slot. Moreover, as the dimensions of the directional and omnidirectional antennas are similar [24] and the storage capacity of commercially available efficient compact supercapacitors [25] is large enough to store the harvested energy over multiple slots $N_{s}$, the form factor and storage capability are not a concern in the proposed $\mathrm{i}^{2} \mathrm{ER}$ architecture. The entire $i^{2} E R$ process can be divided into three phases, as highlighted in Fig. 1 and Table I:

(1) RF-ET over $N_{s}$ slots: Apart form single-hop ET from $\mathcal{A}$ to $\mathcal{R}$ and $\mathcal{S}$ over $N_{s}$ slots, last or $\left(N_{s}\right)$ th slot of this phase 
TABLE I: Description of operations in RF-powered communication with energy and information relaying possibility.

\begin{tabular}{|c|c|c|c|c|c|}
\hline \multirow{2}{*}{\multicolumn{2}{|c|}{ 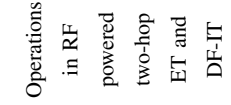 }} & \multicolumn{2}{|c|}{ RF Energy Transfer } & \multicolumn{2}{|c|}{ Information Transfer } \\
\hline & & $\begin{array}{c}\mathcal{A} \text { to } \mathcal{S} \text { and } \mathcal{R} \\
\text { Phase } 1 \text { (a) }\end{array}$ & $\begin{array}{c}\mathcal{R} \text { to } \mathcal{S} \\
\text { Phase } 1 \text { (b) }\end{array}$ & $\begin{array}{c}\mathcal{S} \text { to } \mathcal{A} \text { and } \mathcal{R} \\
\text { Phase } 2\end{array}$ & $\begin{array}{l}\mathcal{R} \text { to } \mathcal{A} \\
\text { Phase } 3\end{array}$ \\
\hline \multicolumn{2}{|r|}{ Duration } & $N_{s}$ slots & 1 slot & 1 slot & 1 slot \\
\hline \multicolumn{2}{|r|}{ Slots \# } & $1,2, \ldots, N_{s}$ & $N_{s}$ & $N_{s}+1$ & $N_{s}+2$ \\
\hline \multirow{4}{*}{ 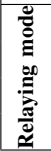 } & No relaying & $\checkmark$ & $x$ & $\checkmark$ & $\mathrm{x}$ \\
\hline & Energy & $\checkmark$ & $\checkmark$ & $\checkmark$ & $x$ \\
\hline & Information & $\checkmark$ & $x$ & $\checkmark$ & $\checkmark$ \\
\hline & $\mathbf{i}^{2} \mathbf{E R}$ & $\checkmark$ & $\checkmark$ & $\checkmark$ & $\checkmark$ \\
\hline
\end{tabular}

also involves two-hop full-duplex ET from $\mathcal{R}$ to $\mathcal{S}$ using $\beta \alpha$ fraction of energy harvested $E_{h_{\mathcal{R}}}$ at $\mathcal{R}$ over $N_{s}$ slots.

(2) IT from $\mathcal{S}$ to $\mathcal{A}$ and $\mathcal{R}$ during $\left(N_{s}+1\right)$ th slot using its energy harvested over $N_{s}$ slots.

(3) IT from $\mathcal{R}$ to $\mathcal{A}$ in $\left(N_{s}+2\right)$ th slot using $\beta(1-\alpha)$ fraction of harvested energy $E_{h_{\mathcal{R}}}$ at $\mathcal{R}$.

Following the summary given in Table I, we note that the four possible relaying modes are: no relaying (NR), ER, IR, and $\mathrm{i}^{2} \mathrm{ER}$. In NR (i.e., neither ER nor IR) mode, indicator variable $\beta$ is set to zero. $\beta=1$ for other modes represents that $\mathcal{R}$ uses $\alpha$ fraction of its harvested energy $E_{h_{\mathcal{R}}}$ for ER and remaining fraction for IR. Thus $\alpha=1$ and $\alpha=0$ respectively represent ER and IR scenarios, whereas $0<\alpha<1$ represents the $i^{2} E R$ mode. Here it is worth noting that, since the energy harvested over a single slot is very low (as shown later in Fig. 3), we have considered $N_{s}>1$ slots for RF-ET because it helps in incorporating the hardware limitations of RF-EH communication [2], [5] while giving insights on the practically realizable rate-constrained sustainable throughput performance (cf. Section VII). Also, in order to have sufficient harvested energy at $\mathcal{R}$ for efficient RF-ET to $\mathcal{S}$, ER is considered only in the $N_{s}$ th slot. Further, due to the usage of two directional antennas at $\mathcal{R}$, the leaked energy from $\mathcal{R}$ in the unintended direction, that could be recycled by its receiver, is neglected.

\section{B. Network Topology}

To avoid blocking of direct ET and IT paths between $\mathcal{A}$ and $\mathcal{S}$, we consider parallel topology [5] for relay placement. Considering static node deployment, $\mathcal{A}$ and $\mathcal{S}$ are respectively located on Euclidean plane with coordinates $(0,0)$ and $\left(d_{\mathcal{A S}}, 0\right)$. Here $d_{\mathcal{A S}}$ is the distance between $\mathcal{A}$ and $\mathcal{S}$. $\mathcal{R}$ is positioned at $\left(x_{\mathcal{R}}, y_{\mathcal{R}_{0}}\right)$, where $y_{\mathcal{R}_{0}}$ is the minimum nonblocking distance [5] from Line-of-Sight (LoS) path between $\mathcal{A}$ and $\mathcal{S}$. However, it is worth mentioning that the proposed $\mathrm{i}^{2} \mathrm{ER}$ model and the analysis hold for any arbitrary relay placement topology ensuring the availability of unaffected direct link between $\mathcal{A}$ and $\mathcal{S}$, e.g., elliptical topology [13].

\section{Channel Model}

All the links are considered independent and experience flat quasi-static Rician block fading [13], where the average channel power gains $\overline{\left|\mathrm{h}_{i j}\right|^{2}}=\mathbb{E}\left[\left|\mathrm{h}_{i j}\right|^{2}\right]=\frac{G_{i} G_{j}}{\left(d_{i j}\right)^{n}}\left(\frac{\lambda}{4 \pi}\right)^{2}$, $\forall i, j=\{\mathcal{A}, \mathcal{R}, \mathcal{S}\}$. Here $G_{i}$ and $G_{j}$ represent antenna gain for transmitting node $i$ and receiving node $j ; \lambda$ is the wavelength of transmitted RF signal; $n$ is path loss exponent; $d_{i j}$ corresponds to distance between nodes $i$ and $j$. Rician channel model helps in incorporating the effect of strong LoS component in RF-ET over short range IT links. Rician fading also includes Rayleigh fading [6]-[10] as its special case. To reduce signalling overhead at energy-constrained $\mathcal{R}$ and $\mathcal{S}$, we assume that knowledge about statistics of channel state information (CSI), instead of instantaneous CSI, for all links is available at $\mathcal{A}$ via pilot signals received from $\mathcal{R}$ and $\mathcal{S}$.

\section{Energy Consumption and RF Harvesting Model}

We assume that $\mathcal{S}$ uses its entire harvested energy for IT. So with unit slot duration $T=1 \mathrm{~s}$, energy or power consumption at $\mathcal{S}$ during IT is: $P_{t_{\mathcal{S}}}+P_{\text {con }}^{\mathrm{tx}}$, where $P_{\text {con }}^{\mathrm{tx}}$ is static power consumption independent of transmit power $P_{t_{\mathcal{S}}}$. Generally $P_{\text {con }}^{\mathrm{tx}} \approx 0$ in cooperative WPCN and SWIPT [6]-[10]. We note that RF energy reception does not consume any power [2], [5]. The consumption at $\mathcal{R}$ for IR is accounted as [26, Table I]: $E_{\mathrm{con}}^{\mathrm{rx}}+R_{0} E_{\mathrm{bit}}^{\mathrm{rx}}$, where $E_{\mathrm{con}}^{\mathrm{rx}}$ is static consumption and $R_{0} E_{\mathrm{bit}}^{\mathrm{rx}}$ is consumption in reception and decoding of $R_{0}$ bits.

The harvested dc power $\mathcal{P}_{h}$ is a nonlinear function of received RF power $\mathcal{P}_{r}$ [5]. To this end, we present a piecewise linear approximation for establishing a relationship between $\mathcal{P}_{h}$ and $\mathcal{P}_{r}$ using a piecewise linear function $\mathcal{L}(\cdot)$. Mathematically, $\mathcal{P}_{h}=\mathcal{L}\left(\mathcal{P}_{r}\right)$ can be defined as:

$\mathcal{P}_{h} \triangleq \begin{cases}0, & \mathcal{P}_{r}<\mathcal{P}_{\mathrm{th}_{1}}, \\ \mathcal{M}_{i} \mathcal{P}_{r}+\mathcal{C}_{i}, & \mathcal{P}_{r} \in\left[\mathcal{P}_{\mathrm{th}_{i}}, \mathcal{P}_{\mathrm{th}_{i+1}}\right], \forall i=1, . ., N,(1) \\ \text { Not applicable, }, & \mathcal{P}_{r}>\mathcal{P}_{\mathrm{th}_{N+1}},\end{cases}$

where $\mathcal{P}_{\text {th }}=\left\{\mathcal{P}_{\text {th }_{i}} \mid 1 \leq i \leq N+1\right\} \mathrm{mW}$ are thresholds on $\mathcal{P}_{r}$ that define the boundaries for $N$ linear pieces with slope $\mathcal{M}=$ $\left\{\mathcal{M}_{i} \mid 1 \leq i \leq N\right\}$ and intercept $\mathcal{C}=\left\{\mathcal{C}_{i} \mid 1 \leq i \leq N\right\} \mathrm{mW}$.

We have used the above approximation $\mathcal{L}(\cdot)$ because this simple linear relationship between $\mathcal{P}_{r}$ and $\mathcal{P}_{h}$ helps in gaining insights on global-optimal harvested energy utilization and proving conditional-unimodality of throughput maximization problem $(P 1)$ in $\alpha$ (cf. Section VI).

\section{DownLink RF ENERgy TRANSFER AND ENERgY RELAYING OVER RICIAN CHANNELS}

The energy signals $\mathbb{Y}_{\mathcal{A R}}$ and $\mathbb{Y}_{\mathcal{A S}}$ received respectively at $\mathcal{R}$ and $\mathcal{S}$ in one slot of ET from $\mathcal{A}$ are given by:

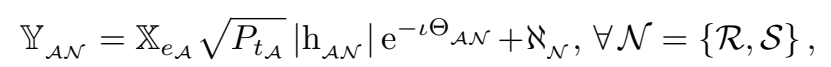

where $\mathbb{X}_{e_{A}}$ is the zero mean and unit variance energy signal transmitted by $\mathcal{A}$, and $P_{t_{\mathcal{A}}}$ is the transmit power of $\mathcal{A}$. $\left|\mathrm{h}_{i j}\right|$ and $\Theta_{i j}=\left(\frac{2 \pi d_{i j}}{\lambda}-\phi_{i j}\right)$ respectively represent the amplitude and phase of the Rician channel fading coefficient for the link between node $i$ and node $j$, where $i, j=\{\mathcal{A}, \mathcal{R}, \mathcal{S}\}$. Here $\frac{2 \pi d_{i j}}{\lambda}$ represents the phase difference due to free space path delay and $\phi_{i j}$ represents the sum of all other phases that include phase weights introduced for synchronization, errors due to the local oscillator variations, excess path phase from obstacles, etc. [27]. Lastly, $\aleph_{i}$ represents zero mean additive white Gaussian noise with variance $\sigma^{2}$ as received at node $i$. 
Using (2) and ignoring EH from noise power [6]-[15], the received power at $\mathcal{R}$ and $\mathcal{S}$ in each slot due to RF-ET from $\mathcal{A}$ is given by (3) where ${ }^{\dagger}$ denotes the complex conjugate.

$\mathcal{P}_{r_{\mathcal{A N}}}=\left|\left(\mathbb{Y}_{\mathcal{A N}}\right)\left(\mathbb{Y}_{\mathcal{A N}}\right)^{\dagger}\right|=P_{t_{\mathcal{A}}}\left|\mathrm{h}_{\mathcal{A N}}\right|^{2}, \forall \mathcal{N}=\{\mathcal{R}, \mathcal{S}\}$.

As the received powers $\mathcal{P}_{r_{\mathcal{A S}}}$ and $\mathcal{P}_{r_{\mathcal{A R}}}$ at $\mathcal{S}$ and $\mathcal{R}$ involve the square of Rician distributed $\left|\mathrm{h}_{\mathcal{A N}}\right|$, they follow noncentral- $\chi^{2}$ distribution with respective Rice factors and means as $\left(K_{\mathcal{A S}}, \mu_{\mathcal{P}_{\mathcal{A S}}}=\frac{P_{t_{\mathcal{A}}} G_{\mathcal{A}} G_{\mathcal{S}}}{\left(d_{\mathcal{A S}}\right)^{n}}\left(\frac{\lambda}{4 \pi}\right)^{2}\right)$ and $\left(K_{\mathcal{A R}}, \mu_{\mathcal{P}_{\mathcal{A R}}}=\frac{P_{t_{\mathcal{A}}} G_{\mathcal{A}} G_{\mathcal{R}}}{\left(d_{\mathcal{A R}}\right)^{n}}\left(\frac{\lambda}{4 \pi}\right)^{2}\right)$. We next discuss the basic probabilistic measures for the received power over Rician channels and then use them for deriving the mean harvested energy at $\mathcal{S}$ due to RF-ET from $\mathcal{A}$, both with and without ER.

\section{A. Basic Properties of Rician Fading Channels}

For Rician fading, the channel power gains follow noncentral- $\chi^{2}$ distribution with two degrees of freedom. Thus, the probability density function (PDF) $f_{\mathcal{P}_{r}}$ of received power $\mathcal{P}_{r}, \forall x \geq 0$, is given by:

$f_{\mathcal{P}_{r}}\left(x, K, \mu_{\mathcal{P}}\right)=\frac{\mathrm{e}^{-\frac{(K+1) x}{\mu_{\mathcal{P}}}-K}}{\mu_{\mathcal{P}}(K+1)^{-1}} \mathbf{I}_{\mathbf{0}}\left(2 \sqrt{\frac{K(K+1) x}{\mu_{\mathcal{P}}}}\right)$,

where $K$ is Rice factor, $\mu_{\mathcal{P}}$ is mean received power, and $\mathbf{I}_{\mathbf{m}}(\cdot)$ is the modified Bessel function of the first kind with order $\mathbf{m}$. The Cumulative Distribution Function $(\mathrm{CDF}) F_{\mathcal{P}_{r}}$ of $\mathcal{P}_{r}$ is:

$$
F_{\mathcal{P}_{r}}\left(x, K, \mu_{\mathcal{P}}\right)=1-Q_{1}\left(\sqrt{2 K}, \sqrt{2(K+1) x / \mu_{\mathcal{P}}}\right),
$$

where $Q_{1}(\cdot, \cdot)$ is first order Marcum Q-function [28]. Moment generating function (MGF) $\Phi_{\mathcal{P}_{r}}$ of $\mathcal{P}_{r}$ can be obtained as:

$$
\begin{aligned}
\Phi_{\mathcal{P}_{r}}\left(\nu, K, \mu_{\mathcal{P}}\right) & \stackrel{(a)}{=}\left(\frac{K+1}{K-\iota \nu \mu_{\mathcal{P}}+1}\right) \mathrm{e}^{\frac{\iota K \nu \mu_{\mathcal{P}}}{K-\iota \nu \mu_{\mathcal{P}}+1}} \\
& \stackrel{(b)}{=}\left(\frac{K+1}{K-\iota \nu \mu_{\mathcal{P}}+1}\right) \mathrm{e}^{\frac{K(K+1)}{K-\iota \nu \mu_{\mathcal{P}}+1}-K} .
\end{aligned}
$$

Here $(a)$ is obtained by using [29, eq. (2.17)] and $(b)$ is obtained after a rearrangement in $(a)$.

\section{B. Single-Hop RF-ET from $\mathcal{A}$ to $\mathcal{S}$ and $\mathcal{R}$}

First we obtain mean harvested power $\overline{\mathcal{P}_{h_{\mathcal{A S}}}}$ at $\mathcal{S}$ and $\overline{\mathcal{P}_{h_{\mathcal{A R}}}}$ at $\mathcal{R}$ in each slot dedicated for RF-ET from $\mathcal{A}$. Using $\mathcal{P}_{h}=$ $\mathcal{L}\left(\mathcal{P}_{r}\right)$ as defined in (1) along with PDF $f_{\mathcal{P}_{r}}$ and CDF $F_{\mathcal{P}_{r}}$ of received power $\mathcal{P}_{r}$ defined in (4) and (5), the PDF $f_{\mathcal{P}_{h}}$ of harvested dc power $\mathcal{P}_{h}$ is obtained as:

$$
f_{\mathcal{P}_{h}}\left(x, K, \mu_{\mathcal{P}}\right) \triangleq \frac{\frac{1}{\mathcal{M}_{j}} f_{\mathcal{P}_{r}}\left(\frac{x-\mathcal{C}_{j}}{\mathcal{M}_{j}}, K, \mu_{\mathcal{P}}\right)}{F_{\mathcal{P}_{r}}\left(\mathcal{P}_{\mathrm{th}_{N+1}}\right)-F_{\mathcal{P}_{r}}\left(\mathcal{P}_{\mathrm{th}_{1}}\right)},
$$

where $x$ satisfies $\mathcal{P}_{\mathrm{th}_{j}} \leq \frac{x-\mathcal{C}_{j}}{\mathcal{M}_{j}} \leq \mathcal{P}_{\mathrm{th}_{j+1}}, \forall j \in 1, \ldots, N$. Thus, using (7), the mean harvested dc powers $\overline{\mathcal{P}_{h_{\mathcal{A N}}}}$ where $\mathcal{N}=$ $\{\mathcal{R}, \mathcal{S}\}$ are derived below:

$\overline{\mathcal{P}_{h_{\mathcal{A N}}}}=\mathbb{E}\left[\mathcal{P}_{h_{\mathcal{A N}}}\right]=\int_{0}^{\infty} x f_{\mathcal{P}_{h_{\mathcal{A N}}}}\left(x, K_{\mathcal{A N}}, \mu_{\mathcal{P}_{\mathcal{A N}}}\right) d x$

$$
\begin{aligned}
& =\sum_{j=1}^{N} \int_{\mathcal{M}_{j} \mathcal{P}_{\mathrm{th}_{j}}+\mathcal{C}_{j}}^{\mathcal{M}_{j} \mathcal{P}_{\mathrm{th}_{j}+1}+\mathcal{C}_{j}} \frac{x\left(K_{\mathcal{A N}}+1\right) \mathrm{e}^{-\frac{\left(K_{\mathcal{A N}}+1\right)\left(x-\mathcal{C}_{j}\right)}{\mathcal{M}_{j} \mathcal{P}_{\mathcal{A N}}}-K_{\mathcal{A N}}}}{\mathcal{M}_{j} \mu_{\mathcal{P}_{\mathcal{A N}}}} \\
& \times \frac{\mathbf{I}_{\mathbf{0}}\left(2 \sqrt{\frac{K_{\mathcal{A N}}\left(K_{\mathcal{A N}}+1\right)\left(x-\mathcal{C}_{j}\right)}{\mathcal{M}_{j} \mu_{\mathcal{P} \mathcal{N}}}}\right)}{F_{\mathcal{P}_{r_{\mathcal{A N}}}\left(\mathcal{P}_{\mathrm{th}_{N+1}}\right)-F_{\mathcal{P}_{r_{\mathcal{N}}}}\left(\mathcal{P}_{\mathrm{th}_{1}}\right)}} d x \\
& \stackrel{(c)}{=} \sum_{k=0}^{\infty} \sum_{j=1}^{N} \int_{\mathcal{M}_{j} \mathcal{P}_{\mathrm{th}_{j}}+\mathcal{C}_{j}}^{\mathcal{M}_{j} \mathcal{P}_{\mathrm{th}_{j+1}}+\mathcal{C}_{j}} \frac{\mathrm{e}^{-\frac{\left(K_{\mathcal{A N}^{\prime}}+1\right)\left(x-\mathcal{C}_{j}\right)}{\mathcal{M}_{j} \mathcal{P}_{\mathcal{A N}}}-K_{\mathcal{A N}}}}{\left(\mathcal{M}_{j} \mu_{\mathcal{P}_{\mathcal{A N}}}\right)^{k+1}(k !)^{2}} \\
& \times \frac{\left(K_{\mathcal{A N}}+1\right)\left[K_{\mathcal{A N}}\left(K_{\mathcal{A N}}+1\right)\left(x-\mathcal{C}_{j}\right)\right]^{k}}{F_{\mathcal{P}_{r_{\mathcal{A N}}}}\left(\mathcal{P}_{\mathrm{th}_{N+1}}\right)-F_{\mathcal{P}_{r_{\mathcal{A N}}}}\left(\mathcal{P}_{\mathrm{th}_{1}}\right)} d x
\end{aligned}
$$

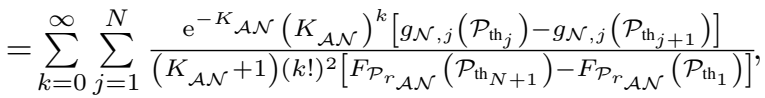

where $g_{\mathcal{N}, j}\left(\mathcal{P}_{\mathrm{th}_{j}}\right) \triangleq \mathcal{C}_{j}\left(K_{\mathcal{A N}}+1\right) \Gamma\left(k+1, \frac{\left(K_{\mathcal{A N}}+1\right) \mathcal{P}_{\mathrm{th}_{j}}}{\mu_{\mathcal{P}_{\mathcal{A N}}}}\right)+$ $\mathcal{M}_{j} \mu_{\mathcal{P}_{\mathcal{N}}} \Gamma\left(k+2, \frac{\left(K_{\mathcal{A N}_{\mathcal{N}}}+1\right) \mathcal{P}_{\mathrm{th}_{j}}}{\mu_{\mathcal{P}_{\mathcal{A N}}}}\right)$. Each term in Taylor series expansion of $\mathbf{I}_{\mathbf{0}}(\cdot)$ used in $(c)$ can be upper bounded as:

$$
\begin{gathered}
\frac{\left(\frac{K_{\mathcal{A N}}\left(K_{\mathcal{A N}}+1\right)\left(x-\mathcal{C}_{j}\right)}{\mathcal{M}_{j} \mu_{\mathcal{P} \mathcal{N}}}\right)^{k}}{(k !)^{2}} \stackrel{(d)}{\lesssim} \frac{\left(4 K_{\mathcal{A N}}\left(K_{\mathcal{A N}}+1\right)\right)^{k}}{(k !)^{2}} \\
\stackrel{(e)}{\leq} \frac{\left(\frac{2 \mathrm{e}\left(K_{\mathcal{A N}}+1\right)}{k}\right)^{2 k}}{2 \pi k} \leq\left(\frac{2 \mathrm{e}\left(K_{\mathcal{A N}}+1\right)}{k}\right)^{2 k} .
\end{gathered}
$$

Here $(d)$ is obtained by knowing that generally $\left(x-\mathcal{C}_{j}\right) \leq$ $4 \mathcal{M}_{j} \mu_{\mathcal{P}_{\mathcal{A N}}}$, as from (5) $\operatorname{Pr}\left(\mathcal{P}_{r}>4 \mu_{\mathcal{P}}\right)<0.009, \forall K \geq 1$, and $(e)$ is obtained using the Stirling's approximation [30]: $j ! \approx$ $\sqrt{2 \pi} \mathrm{e}^{-j} j^{j+\frac{1}{2}}$. From (9) we note that the contribution of higher order terms $k>\ln \left(\frac{1}{\epsilon}\right)\left[2 \boldsymbol{W}_{\mathbf{0}}\left(\frac{\log \left(\frac{1}{\epsilon}\right)}{4 \mathrm{e}\left(K_{\mathcal{A N}}+1\right)}\right)\right]^{-1}$ is very less than $\epsilon$, where $\epsilon \ll 1$ and $W_{\mathbf{0}}(x)$ is the Lambert function (principal branch) [31]. However, in general for high Rice factor $K_{\mathcal{A N}} \geq 10$, even considering only first $k=\mathrm{e}\left(K_{\mathcal{A N}}+1\right)$ summands provides a very tight match to the infinite series because the product term $\frac{x\left(K_{\mathcal{A N}}+1\right)}{\mathcal{M}_{j} \mu_{\mathcal{P}_{\mathcal{A N}}}} \mathrm{e}^{-K_{\mathcal{A N}}} \mathrm{e}^{-\frac{\left(K_{\mathcal{A N}}+1\right)\left(x-\mathcal{C}_{j}\right)}{\mathcal{M}_{j} \mu_{\mathcal{P}} \mathcal{A N}}}$ decays very fast with increasing $K_{\mathcal{A N}}$. This has been numerically validated later in Fig. 3 where (8) is shown to be equivalent to the sum of first 30 summands.

So, with $T=1 \mathrm{~s}$ as slot duration, mean harvested energy $E_{h_{\mathcal{R}}}$ at $\mathcal{R}$ via RF-ET from $\mathcal{A}$ over $N_{s}$ slots is: $E_{h_{\mathcal{R}}} \triangleq$ $\overline{\mathcal{P}_{h_{\mathcal{A R}}}} N_{s}$. Similarly for NR and IR (i.e., no ER modes), the mean harvested energy at $\mathcal{S}$ is: $E_{h_{\mathcal{S}}}^{\mathrm{noER}} \triangleq \overline{\mathcal{P}_{h_{\mathcal{A S}}}} N_{s}$. However for ER and i ${ }^{2}$ ER modes, mean harvested energy at $\mathcal{S}$ via single hop ET from $\mathcal{A}$ over $N_{s}-1$ slots is: $E_{h_{\mathcal{S}}}^{N_{s}-1} \triangleq \overline{\mathcal{P}_{h_{\mathcal{A}}}}\left(N_{s}-1\right)$. Next we find energy harvested at $\mathcal{S}$, via two-hop ET from $\mathcal{R}$, in the last slot of RF-ET phase in ER and $\mathrm{i}^{2} \mathrm{ER}$ modes.

\section{Mean Energy Harvested in $N_{s}$ th Slot due to Two-Hop ER}

The received energy signal $\mathbb{Y}_{\mathcal{R S}}$ at $\mathcal{S}$ in the $N_{s}$ th slot due to ER from $\mathcal{R}$ is given by:

$$
\mathbb{Y}_{\mathcal{R S}}=\mathbb{X}_{\mathcal{R}_{\mathcal{R}}} \sqrt{\beta \alpha P_{t_{\mathcal{R}}}}\left|\mathrm{h}_{\mathcal{R} \mathcal{S}}\right| \mathrm{e}^{-\iota \Theta_{\mathcal{R S}}}+\aleph_{\mathcal{S}},
$$


where $\mathbb{X}_{e_{\mathcal{R}}}$ is the zero mean, unit variance energy signal transmitted by $\mathcal{R}$ using its energy harvested $E_{h_{\mathcal{R}}}$ over $N_{s}$ slots and $P_{t_{\mathcal{R}}}$ is the transmit power of $\mathcal{R}$. $\beta$ is indicator variable for relaying with $\alpha$ as fraction of $E_{h_{\mathcal{R}}}$ allocated for ER. $\left|\mathrm{h}_{\mathcal{R S}}\right|$ and $\Theta_{\mathcal{R S}}=\left(\frac{2 \pi d_{\mathcal{R S}}}{\lambda}-\phi_{\mathcal{R S}}\right)$ respectively represent the amplitude and phase of Rician fading coefficient for $\mathcal{R}$-to- $\mathcal{S}$ link. So, we note that if ER takes place from $\mathcal{R}$ to $\mathcal{S}$, i.e., $\alpha>0$, then $\mathcal{S}$ receives two energy signals $\mathbb{Y}_{\mathcal{A S}}$ and $\mathbb{Y}_{\mathcal{R S}}$ in the $N_{s}$ th slot and the received power at $\mathcal{S}$ in the $N_{s}$ th slot is different from (3). Thus using (3) and (10), the random received power $\mathcal{P}_{r_{\mathcal{S}}}^{\text {hhop }}$ at $\mathcal{S}$ in $\left(N_{s}\right)$ th slot of ER phase, due to vector addition of energy signals received from $\mathcal{A}$ and $\mathcal{R}$, is given by [5, eq. (14)]:

$$
\begin{aligned}
& \mathcal{P}_{r_{\mathcal{S}}}^{2 \text { hop }}=\left|\left(\mathbb{Y}_{\mathcal{A S}}+\mathbb{Y}_{\mathcal{R S}}\right)\left(\mathbb{Y}_{\mathcal{A S}}+\mathbb{Y}_{\mathcal{R S}}\right)^{\dagger}\right|=\left|\mathbb{Y}_{\mathcal{A S}}\right|^{2}+\left|\mathbb{Y}_{\mathcal{R S}}\right|^{2}
\end{aligned}
$$

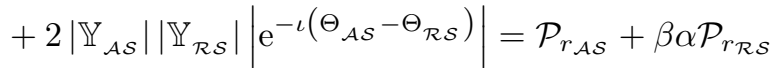

$$
\begin{aligned}
& +2 \sqrt{\mathcal{P}_{r_{\mathcal{A S}}} \beta \alpha \mathcal{P}_{r_{\mathcal{R}}}} \mathrm{e}^{-\overline{\psi^{2}}} \cos \left(\frac{2 \pi\left(d_{\mathcal{A S}}-d_{\mathcal{R S}}\right)}{\lambda}\right),
\end{aligned}
$$

where $\overline{\psi^{2}}$ is the root mean square phase error term incorporating the errors due to the local oscillator variations, excess path phase from obstacles, etc. [27]. $\overline{\psi^{2}}$ is in radians and $\mathrm{e}^{-\overline{\psi^{2}}}$ is unit-less. Here vector addition of RF signals of same frequency received from $\mathcal{A}$ and $\mathcal{R}$ is considered because these energy waves can combine constructive or destructively depending on their respective in-phase or out-of-phase addition [5]. So, mean received power $\overline{\mathcal{P}_{r_{\mathcal{S}}}^{\text {hop }}}=\mathbb{E}\left[\mathcal{P}_{r_{\mathcal{S}}}^{\text {hop }}\right]$, obtained using linearity of expectation and independence of $\mathcal{P}_{r_{\mathcal{A S}}}$ and $\mathcal{P}_{r_{\mathcal{R} S}}$, is:

$$
\begin{aligned}
& \overline{\mathcal{P}_{r_{\mathcal{S}}}^{2 \text { hop }}}=\mathbb{E}\left[\mathcal{P}_{r_{\mathcal{A S}}}\right]+\beta \alpha \mathbb{E}\left[\mathcal{P}_{r_{\mathcal{R S}}}\right]+2 \sqrt{\beta \alpha} \mathbb{E}\left[\sqrt{\mathcal{P}_{r_{\mathcal{A S}}}}\right] \\
& \times \mathbb{E}\left[\sqrt{\mathcal{P}_{r_{\mathcal{R S}}}}\right] \mathrm{e}^{-\overline{\psi^{2}}} \cos \left(\frac{2 \pi\left(d_{\mathcal{A S}}-d_{\mathcal{R S}}\right)}{\lambda}\right)=\mu_{\mathcal{P}_{\mathcal{A S}}}+\beta \alpha \mu_{\mathcal{P}_{\mathcal{R S}}} \\
& +2 \sqrt{\beta \alpha} \mu_{\sqrt{\mathcal{P}_{\mathcal{A S}}}} \mu_{\sqrt{\mathcal{P}_{\mathcal{R S}}}} \mathrm{e}^{-\overline{\psi^{2}}} \cos \left(\frac{2 \pi\left(d_{\mathcal{A S}}-d_{\mathcal{R S}}\right)}{\lambda}\right),(12)
\end{aligned}
$$

where $\mu_{\mathcal{P}_{\mathcal{R S}}} \triangleq \frac{\left(E_{h_{\mathcal{R}}}+E_{i_{\mathcal{R}}}\right) G_{t_{\mathcal{R}}} G_{\mathcal{S}}}{\left(d_{\mathcal{R} \mathcal{S}}\right)^{n}}\left(\frac{\lambda}{4 \pi}\right)^{2}$ with $\mu_{\mathcal{P}_{\mathcal{A S}}}=$ $\frac{P_{t_{\mathcal{A}}} G_{\mathcal{A}} G_{\mathcal{S}}}{\left(d_{\mathcal{A S}}\right)^{n}}\left(\frac{\lambda}{4 \pi}\right)^{2}$ and $E_{h_{\mathcal{R}}}$ as defined in Section III-B. Here $E_{i_{\mathcal{R}}}$ is the unused harvested energy which is available as the residual or initial energy at $\mathcal{R}$ when NR mode was selected in previous transmission block. The accumulated energy $E_{i_{\mathcal{R}}}$ is zero when any other relaying mode is chosen. $\mu_{\sqrt{\mathcal{P}_{\mathcal{A S}}}}$ and $\mu_{\sqrt{\mathcal{P}_{\mathcal{R S}}}}$ in (12) are respectively obtained by substituting $\mu_{\mathcal{P}_{\mathcal{A S}}}$ and $\mu_{\mathcal{P}_{r_{\mathcal{R}} \mathcal{S}}}$ in place of $\mu_{\mathcal{P}}$ in (13) providing $\mathbb{E}\left[\sqrt{\mathcal{P}_{r}}\right]$.

$$
\mu_{\sqrt{\mathcal{P}_{r}}} \triangleq \frac{\mathrm{e}^{-\frac{K}{2}}}{2} \sqrt{\frac{\pi \mu_{\mathcal{P}}}{K+1}}\left[(K+1) \mathbf{I}_{\mathbf{0}}\left(\frac{K}{2}\right)+K \mathbf{I}_{\mathbf{1}}\left(\frac{K}{2}\right)\right] .
$$

The above expression is obtained by finding the mean of square-root of random variable $\mathcal{P}_{r}$ following noncentral- $\chi^{2}$ distribution with two degrees of freedom.

Using (12) and (13), the mean harvested power at $\mathcal{S}$ due to ER in last slot of RF-ET phase is given by $\overline{\mathcal{P}_{h_{\mathcal{S}}}^{2 \text { hop }}}=\mathcal{L}\left(\overline{\mathcal{P}_{r_{\mathcal{S}}}^{2 \text { hop }}}\right)$. The total energy harvested at $\mathcal{S}$ in $N_{s}$ slots for ER and $\mathrm{i}^{2} \mathrm{ER}$ modes is: $E_{h_{\mathcal{S}_{\text {tot }}}^{\mathrm{ER}}}^{\mathrm{E}}=E_{h_{\mathcal{S}}}^{N_{s}-1}+\overline{\mathcal{P}_{h_{\mathcal{S}}}^{2 h o p}}$. For NR and IR, $\overline{\mathcal{P}_{h_{\mathcal{S}}}^{2 \text { hop }}}=$ $\overline{\mathcal{P}_{h_{\mathcal{A S}}}}$, which implies that $E_{h_{\mathcal{S}}}^{\mathrm{noER}}=\overline{\mathcal{P}_{h_{\mathcal{A S}}}} N_{s}$.

\section{DF RELAY ASSISTED COMMUNICATION OVER RICIAN Channels WiTh DiRECT LiNK}

For the RF-powered IT with $T=1 \mathrm{~s}$, the transmit powers $P_{t_{\mathcal{R}}}=E_{h_{\mathcal{R}}}^{u}+E_{i_{\mathcal{R}}}$ and $P_{t_{\mathcal{S}}}=E_{h_{\mathcal{S}}}^{u}$ of $\mathcal{R}$ and $\mathcal{S}$ depend on their usable harvested energies $E_{h_{\mathcal{R}}}^{u}=\beta\left[(1-\alpha) \overline{\mathcal{P}_{h_{\mathcal{A R}}}} N_{s}-P_{\mathrm{con}}^{\mathrm{tx}}\right.$ $\left.-E_{\text {con }}^{\mathrm{rx}}-R_{0} E_{\mathrm{bit}}^{\mathrm{rx}}\right]^{+}$and $E_{h_{\mathcal{S}}}^{u}=\left[E_{h_{\mathcal{S}_{\text {tot }}}^{\mathrm{ER}}}-P_{\text {con }}^{\mathrm{tx}}\right]^{+}$for IT, as discussed in Sections II-D and III. Here $[x]^{+}=\max \{0, x\}$. With $P_{t_{\mathcal{S}}}$ as transmit power of $\mathcal{S}$ and $\mathbb{X}_{i_{\mathcal{S}}}$ as zero mean and unit variance information signal, the received signals at $\mathcal{R}$ and $\mathcal{A}$ due to uplink IT from $\mathcal{S}$ in $\left(N_{s}+1\right)$ th are:

$\mathbb{Y}_{\mathcal{S N}}=\mathbb{X}_{i_{\mathcal{S}}} \sqrt{P_{t_{\mathcal{S}}}}\left|\mathrm{h}_{\mathcal{S N}}\right| \mathrm{e}^{-\iota \Theta_{\mathcal{S N}}}+\aleph_{\mathcal{N}}, \forall \mathcal{N}=\{\mathcal{A}, \mathcal{R}\}$.

From the received information symbol $\mathbb{Y}_{\mathcal{S R}}, \mathcal{R}$ forwards the decoded zero mean, unit power signal $\mathbb{X}_{i_{\mathcal{S}}}$ to $\mathcal{A}$ using its harvested energy with transmit power $P_{t_{\mathcal{R}}}$ in a two-hop halfduplex fashion in the $\left(N_{s}+2\right)$ th slot. The RF signal, thus received at $\mathcal{A}$ is given by:

$$
\mathbb{Y}_{\mathcal{R A}}=\widehat{\mathbb{X}_{i_{\mathcal{S}}}} \sqrt{P_{t_{\mathcal{R}}}}\left|\mathrm{h}_{\mathcal{R} \mathcal{A}}\right| \mathrm{e}^{-\iota \Theta_{\mathcal{R A}}+\aleph_{\mathcal{A}}} .
$$

For Rician fading channel model, the instantaneous signal-tonoise ratio (SNR) $\gamma=\frac{\mathcal{P}_{r}}{\sigma^{2}}$ follows the weighted noncentral- $\chi^{2}$ distribution with two degrees of freedom. Using (14) and (15), the received SNRs $\gamma_{\mathcal{S R}}, \gamma_{\mathcal{R} \mathcal{A}}$, and $\gamma_{\mathcal{S A}}$ of $\mathcal{S}$-to- $\mathcal{R}, \mathcal{R}$-to- $\mathcal{A}, \mathcal{S}$ to- $\mathcal{A}$ links, respectively, are given by:

$$
\gamma_{\mathcal{N}_{1} \mathcal{N}_{2}}=\frac{\mathcal{P}_{r_{\mathcal{N}_{1} \mathcal{N}_{2}}}}{\sigma^{2}}=\frac{P_{t_{\mathcal{N}_{1}}}}{\sigma^{2}}\left|h_{\mathcal{N}_{1} \mathcal{N}_{2}}\right|^{2},
$$

$\forall\left(\mathcal{N}_{1}, \mathcal{N}_{2}\right)=\{(\mathcal{S}, \mathcal{R}),(\mathcal{R}, \mathcal{A}),(\mathcal{S}, \mathcal{A})\}$. Due to the availability of the direct link and MRC of received signals at $\mathcal{A}$, the received SNR at $\mathcal{A}$ involves the sum of $\gamma_{\mathcal{S A}}$ and $\gamma_{\mathcal{R A}}$. Next we derive the distribution of this sum to obtain the closed-form expressions for outage probability and normalized throughput at $\mathcal{A}$ due to IT from $\mathcal{S}$ using harvested energy.

\section{A. Sum of Two Weighted Noncentral- $\chi^{2}$ Random Variables}

The distribution of sum of two positive weighted noncentral$\chi^{2}$ random variables can be obtained in terms of Laguerre expansions [32, eq. (3.5)]. However, due to the involvement of complicated recursions in PDF and CDF expressions, its usage has been limited and an integral definition was used in [13]. Here, we present simple expressions for PDF and CDF of this sum by using series expansion of exponential function. The PDF $f_{\mathcal{P}_{r_{1}}+\mathcal{P}_{r_{2}}}$ of sum of two positive weighted noncentral$\chi^{2}$ random variables $\mathcal{P}_{r_{1}}$ and $\mathcal{P}_{r_{2}}$ having respective Rice factor and mean as $\left(K_{1}, \mu_{\mathcal{P}_{1}}\right)$ and $\left(K_{2}, \mu_{\mathcal{P}_{2}}\right)$ is given by:

$$
\begin{aligned}
& f_{\mathcal{P}_{r_{1}}+\mathcal{P}_{r_{2}}}\left(x, K_{1}, \mu_{\mathcal{P}_{1}}, K_{2}, \mu_{\mathcal{P}_{2}}\right) \\
& =\frac{1}{2 \pi} \int_{-\infty}^{\infty} \mathrm{e}^{-\iota \nu x} \Phi_{\mathcal{P}_{r_{1}}}\left(\nu, K_{1}, \mu_{\mathcal{P}_{1}}\right) \Phi_{\mathcal{P}_{r_{2}}}\left(\nu, K_{2}, \mu_{\mathcal{P}_{2}}\right) d \nu \\
& \stackrel{(f)}{=} \frac{1}{2 \pi} \int_{-\infty}^{\infty} \sum_{j=0}^{\infty} \sum_{k=0}^{\infty} \frac{\left(\frac{K_{1}\left(K_{1}+1\right)}{K_{1}-\iota \nu \mu_{\mathcal{P}_{1}}+1}\right)^{j+1}\left(\frac{K_{2}\left(K_{2}+1\right)}{K_{2}-\iota \nu \mu_{\mathcal{P}_{2}}+1}\right)^{k+1} d \nu}{j ! k ! K_{1} K_{2} \mathrm{e}^{K_{1}+K_{2}+\iota \nu x}} \\
& \stackrel{(g)}{=} \sum_{j=0}^{\infty} \sum_{k=0}^{\infty}\left(\frac{K_{1}\left(K_{1}+1\right)}{\mu_{\mathcal{P}_{1}}}\right)^{j+1}\left(\frac{K_{2}\left(K_{2}+1\right)}{\mu_{\mathcal{P}_{2}}}\right)^{k+1} x^{j+k+1}
\end{aligned}
$$




$$
\times \frac{{ }_{1} F_{1}\left(j+1 ; j+k+2 ;\left(\frac{K_{2}+1}{\mu_{\mathcal{P}_{2}}}-\frac{K_{1}+1}{\mu_{\mathcal{P}_{1}}}\right) x\right)}{K_{1} K_{2} j ! k ! \Gamma(j+k+2) \mathrm{e}^{K_{1}+\frac{\left(K_{2}+1\right) x}{\mu_{\mathcal{P}_{2}}}+K_{2}}},
$$

where $(f)$ is obtained by substituting series expansion of $\mathrm{e}^{x}=\sum_{k=0}^{\infty} \frac{x^{k}}{k !}$ in (6). $(g)$ is obtained by using a readily available result given by [28, eq. (3.384.7)] in $(f) .{ }_{1} F_{1}(a ; b ; x)=$ $\frac{\Gamma(b) \int_{0}^{1} \mathrm{e}^{x t} t^{a-1}(1-t)^{b-a-1} d t}{\Gamma(b-a) \Gamma(a)}=\sum_{k=0}^{\infty} \frac{(a)_{k} x^{k}}{(b)_{k} k !}$ is the confluent hypergeometric function of the first kind [28], and $(a)_{k},(b)_{k}$ are the Pochhammer symbols defined as: $(a)_{k}=\frac{\Gamma(a+k)}{\Gamma(a)} . \Gamma(x)=$ $\int_{0}^{\infty} t^{x-1} \mathrm{e}^{-t} d t$ is the gamma function. As $\mathrm{e}^{-K_{i}}\left|\mathrm{e}^{\frac{K_{i}\left(K_{i}+1\right)}{K_{i}-\iota \nu \mu_{\mathcal{P}_{i}}+1}}\right|<$ $1 \forall i=\{1,2\}, \mathrm{e}^{\frac{K_{i}\left(K_{i}+1\right)}{K_{i}-\iota \nu \mu_{\mathcal{P}_{i}}+1}-K_{i}}$ and $\frac{\mathrm{e}^{-K_{i}}}{j !}\left(\frac{K_{i}\left(K_{i}+1\right)}{K_{i}-\iota \nu \mu_{\mathcal{P}_{i}}+1}\right)^{j}$ are integrable, and we can swap the integration and double summation [30, Theorem 16.7] in (f) to obtain the result given by (17). Further, the Lagrange remainder $\mathfrak{R}_{j}$ in $j$ term finite Taylor series approximation of exponential function $\mathrm{e}^{\frac{K_{i}\left(K_{i}+1\right)}{K_{i}-\iota \nu \mu_{\mathcal{P}_{i}}+1}-K_{i}}$ with $z \in\left(0, \frac{K_{i}\left(K_{i}+1\right)}{K_{i}-\iota \nu \mu_{\mathcal{P}_{i}}+1}\right)$ is given by:

$$
\begin{aligned}
& \left|\Re_{j}\right|=\mathrm{e}^{-K_{i}}\left|\frac{\mathrm{e}^{z}}{(j+1) !}\left(\frac{K_{i}\left(K_{i}+1\right)}{K_{i}-\iota \nu \mu_{\mathcal{P}_{i}}+1}\right)^{j+1}\right| \\
& \stackrel{(h 1)}{<} \frac{\left(K_{i}\right)^{j+1}}{(j+1) !} \stackrel{(h 2)}{\leq} \frac{\left(\mathrm{e} K_{i}\right)^{j+1}}{\sqrt{2 \pi}(j+1)^{j+1+\frac{1}{2}}} \stackrel{(h 3)}{<}\left(\frac{\mathrm{e} K_{i}}{j+1}\right)^{j+1},
\end{aligned}
$$

where $(h 1)$ is obtained by using $z<\frac{K_{i}\left(K_{i}+1\right)}{K_{i}-\iota \nu \mu_{\mathcal{P}_{i}}+1}$ and $\left(\nu \mu_{\mathcal{P}_{i}}\right)^{2}$ $>0 ;(h 2)$ is obtained using the Stirling's approximation [30]: $j ! \approx \sqrt{2 \pi} \mathrm{e}^{-j} j^{j+\frac{1}{2}}$; and (h3) holds as $\sqrt{2 \pi j}>1$. Using (18), the minimum numbers of terms $j^{*}$ to ensure that the Lagrange remainder $\mathfrak{R}_{j^{*}}$ after considering $j^{*}$ terms is less than $\epsilon$, i.e., $\left|\Re_{j^{*}}\right|<\epsilon$, is given by: $j^{*} \geq \log \left(\frac{1}{\epsilon}\right)\left[\boldsymbol{W}_{\mathbf{0}}\left(\frac{\log \left(\frac{1}{\epsilon}\right)}{e K_{i}}\right)\right]^{-1}-1$. To gain further insight, we note that for high Rice factor values $K_{i} \geq 10, j^{*} \approx \mathrm{e} K_{i}=2.72 K_{i}$.

PDF of $\mathcal{P}_{r_{1}}+\mathcal{P}_{r_{2}}$ for Rayleigh fading case $\left(K_{1}=K_{2}=0\right)$ can be obtained in simple form from (17) by considering single term $j=k=0$ in double summation with $K_{1}, K_{2} \rightarrow 0$ :

$$
f_{\mathcal{P}_{r_{1}}+\mathcal{P}_{r_{2}}}^{\text {Ray }}\left(x, \mu_{\mathcal{P}_{1}}, \mu_{\mathcal{P}_{2}}\right)=\frac{\mathrm{e}^{-\frac{x}{\mu_{\mathcal{P}_{1}}}}-\mathrm{e}^{-\frac{x}{\mu_{\mathcal{P}_{2}}}}}{\mu_{\mathcal{P}_{1}}-\mu_{\mathcal{P}_{2}}}, \forall x \geq 0 .
$$

The CDF of $\mathcal{P}_{r_{1}}+\mathcal{P}_{r_{2}}$, obtained using series representation of ${ }_{1} F_{1}(a ; b ; x)=\sum_{i=0}^{\infty} \frac{(a)_{i} x^{i}}{(b)_{i} i !}$ in $(17)$, is given by:

$$
\begin{gathered}
F_{\mathcal{P}_{r_{1}}+\mathcal{P}_{r_{2}}}\left(x, K_{1}, \mu_{\mathcal{P}_{1}}, K_{2}, \mu_{\mathcal{P}_{2}}\right)=\sum_{i=0}^{\infty} \sum_{j=0}^{\infty} \sum_{k=0}^{\infty} \frac{K_{1}^{j} K_{2}^{k}(j+1)_{i}}{\mathrm{e}^{K_{1}+K_{2}}} \\
\times\left(\frac{K_{1}+1}{\mu_{\mathcal{P}_{1}}}\right)^{j+1}\left(\frac{K_{2}+1}{\mu_{\mathcal{P}_{2}}}\right)^{-i-j-1}\left(\frac{K_{2}+1}{\mu_{\mathcal{P}_{2}}}-\frac{K_{1}+1}{\mu_{\mathcal{P}_{1}}}\right)^{i} \\
\times \frac{\left[\Gamma(i+j+k+2)-\Gamma\left(i+j+k+2, \frac{\left(K_{2}+1\right) x}{\mu_{\mathcal{P}_{2}}}\right)\right]}{(j+k+2)_{i} i ! j ! k ! \Gamma(j+k+2)} .
\end{gathered}
$$

$\Gamma(a, x)=\int_{x}^{\infty} t^{a-1} \mathrm{e}^{-t} d t$ is upper incomplete gamma function. As with $a=j+1<j+k+2=b, \frac{(a)_{i} x^{i}}{(b)_{i} i !} \leq \frac{x^{i}}{i !}$, we note that $j^{*}$ for the Lagrange remainder $\Re_{j^{*}}$ in the Taylor series expansion of ${ }_{1} F_{1}(a ; b ; x)$ to be less than $\epsilon$, is lesser than in case of Lagrange remainder in the Taylor series expansion of exponential function e $(x)$. Hence, although (20) involves three series, each of the three infinite sum-terms converge very quickly. Also we show later in Fig. 4 that practically this CDF reduces to a finite sum with only 30 summands in each series.

Similarly using (19), the expression for CDF of $\mathcal{P}_{r_{1}}+\mathcal{P}_{r_{2}}$ for Rayleigh fading channels is:

$$
F_{\mathcal{P}_{r_{1}}+\mathcal{P}_{r_{2}}}^{\text {Ray }}\left(x, \mu_{\mathcal{P}_{1}}, \mu_{\mathcal{P}_{2}}\right)=1-\frac{\mu_{\mathcal{P}_{1}} e^{-\frac{x}{\mu_{\mathcal{P}_{1}}}}-\mu_{\mathcal{P}_{2}} e^{-\frac{x}{\mu_{\mathcal{P}_{2}}}}}{\mu_{\mathcal{P}_{1}}-\mu_{\mathcal{P}_{2}}} .
$$

Using (19) and (21), the $\mathrm{i}^{2} \mathrm{ER}$ performance over Rayleigh fading channels can be investigated.

\section{B. Outage Analysis for RF-powered DF-IR with MRC}

The outage probability $p_{\text {out }}$ is the probability that the data rate received at $\mathcal{A}$ during IT (and IR) phase (of duration 1 or 2 slots depending on relaying mode) falls below a spectral efficiency threshold $R_{0}$ bits/sec/Hz or bps/Hz. Considering half-duplex DF-IT from $\mathcal{S}$ to $\mathcal{A}$ via $\mathcal{R}$ with MRC at $\mathcal{A}$ due to direct link availability, the outage probability $p_{\text {out }}^{\mathrm{IR}}$ for IR or $\mathrm{i}^{2} \mathrm{ER}$ over the Rician channels is given by:

$$
\begin{aligned}
& p_{\text {out }}^{\mathrm{IR}} \stackrel{(\text { i1 })}{=} \operatorname{Pr}\left(\frac{1}{2} \log _{2}\left(1+\min \left\{\gamma_{\mathcal{S R}}, \gamma_{\mathcal{R A}}+\gamma_{\mathcal{S A}}\right\}\right)<R_{0}\right) \\
& =\operatorname{Pr}\left(\min \left\{\gamma_{\mathcal{S R}}, \gamma_{\mathcal{R} \mathcal{A}}+\gamma_{\mathcal{S A}}\right\}<2^{2 R_{0}}-1\right) \\
& =1-\operatorname{Pr}\left(\gamma_{\mathcal{S R}}>2^{2 R_{0}}-1\right) \operatorname{Pr}\left(\gamma_{\mathcal{R A}}+\gamma_{\mathcal{S A}}>2^{2 R_{0}}-1\right) \\
& \stackrel{(i 2)}{=} 1-\left[1-F_{\mathcal{P}_{r_{\mathcal{S}}}}\left(2^{2 R_{0}}-1, K_{\mathcal{S R}}, \frac{\mu_{\mathcal{P}_{\mathcal{S R}}}}{\sigma^{2}}\right)\right][1-
\end{aligned}
$$

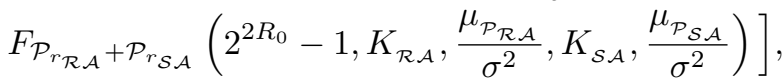

where $(i 1)$ is due to half-duplex DF-IR with MRC [13]. $(i 2)$ is obtained using (16) and fact that $\gamma_{\mathcal{N}_{1}, \mathcal{N}_{2}}$ follows noncentral- $\chi^{2}$ distribution with two degrees of freedom, Rice factor $K_{\mathcal{N}_{1} \mathcal{N}_{2}}$, and mean $\frac{\mu_{\mathcal{P}_{\mathcal{N}_{1} \mathcal{N}_{2}}}}{\sigma^{2}}=\frac{P_{t_{\mathcal{N}_{1}}} G_{\mathcal{N}_{1}} G_{\mathcal{N}_{2}}}{\left(d_{\mathcal{N}_{1} \mathcal{N}_{2}}\right)^{n} \sigma^{2}}\left(\frac{\lambda}{4 \pi}\right)^{2}, \forall\left(\mathcal{N}_{1}, \mathcal{N}_{2}\right)=$ $\{(\mathcal{S}, \mathcal{R}),(\mathcal{R}, \mathcal{A}),(\mathcal{S}, \mathcal{A})\}$. So pout in $(22)$ can be obtained in closed-form by using CDFs defined in (5) and (20) with appropriate arguments as mentioned in (22). Also, using (21) along with $K=0$ in (5) and (22), the outage probability $p_{\text {out }}^{\text {IR,Ray }}$ for IR over Rayleigh fading channels is given by:

$$
\begin{aligned}
p_{\text {out }}^{\text {IR,Ray }}=1- & \frac{1}{\mu_{\mathcal{P}_{\mathcal{R} \mathcal{A}}}-\mu_{\mathcal{P}_{\mathcal{S A}}}}\left[\mu_{\mathcal{P}_{\mathcal{R A}}} \mathrm{e}^{-\left(2^{2 R_{0}}-1\right)\left(\frac{\sigma^{2}}{\mu_{\mathcal{P}_{\mathcal{R} \mathcal{A}}}}+\frac{\sigma^{2}}{\mu_{\mathcal{P} \mathcal{S} \mathcal{R}}}\right)}\right. \\
& \left.-\mu_{\mathcal{P}_{\mathcal{S} \mathcal{A}}} \mathrm{e}^{-\left(2^{2 R_{0}}-1\right)\left(\frac{\sigma^{2}}{\mu_{\mathcal{P} \mathcal{A}}}+\frac{\sigma^{2}}{\mu_{\mathcal{P} \mathcal{R}}}\right)}\right]
\end{aligned}
$$

Further, for no IR cases, i.e., NR and ER modes, $p_{\text {out }}=p_{\text {out }}^{\text {noIR }}$, obtained using (5), is defined below:

$$
p_{\text {out }}^{\text {noIR }}=1-Q_{1}\left(\sqrt{2 K_{\mathcal{S A}}}, \sqrt{\frac{2\left(K_{\mathcal{S A}}+1\right)\left(2^{R_{0}-1}\right) \sigma^{2}}{\mu_{\mathcal{P} \mathcal{A}}}}\right) .
$$




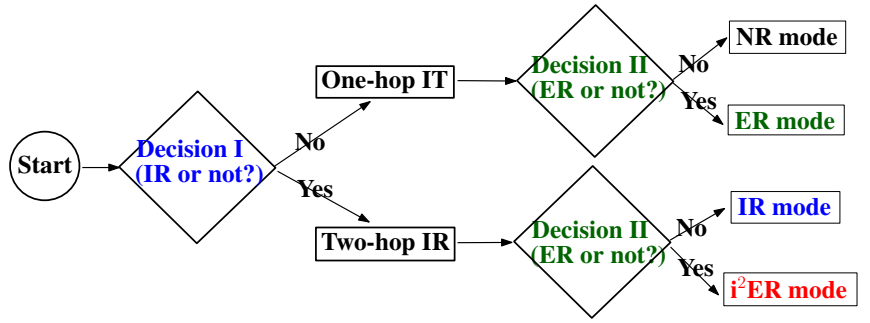

Fig. 2: Decision tree for optimal relaying mode selection policy.

\section{Achievable Normalized Throughput in RF-powered $i^{2} E R$}

Normalized throughput $\bar{\tau}$ is the amount of successfully transmitted data per unit time in each communication slot. Considering a delay-limited scenario with rate constraint (outage threshold) of $R_{0} \mathrm{bps} / \mathrm{Hz}$ at $\mathcal{A}$ for IT phase of 1 or 2 slots (Table I), $\bar{\tau}$ for different relaying modes is:

$\bar{\tau} \triangleq \begin{cases}\frac{R_{0}\left(1-p_{\text {out }}^{\text {nolR }}\right)}{N_{s}+1}, & \mathrm{NR}(\beta=0) \text { and } \operatorname{ER}(\beta=\alpha=1) \\ \frac{2 R_{0}\left(1-p_{\text {out }}^{\mathrm{IR}}\right)}{N_{s}+2}, & \mathrm{IR}(\beta=1, \alpha=0) \text { and } \mathrm{i}^{2} \operatorname{ER}(\beta=1) .\end{cases}$

\section{Optimal Mode Selection at RF-EH Relay}

We now discuss the insights on which mode to choose among NR, ER, IR, and $\mathrm{i}^{2}$ ER. This optimal mode selection policy for efficient outage performance basically involves two main decision making: (i) Two-hop IT with IR or single-hop IT without IR (Section $V$ - $B$ ), and (ii) RF-powered $\mathcal{S}$-to- $\mathcal{A}$ IT with ER or without ER (Section V-A). Fig. 2 summarizes the decision making process in the optimal relaying mode selection policy. Subsequently, we derive conditions for the improved performance of cooperative ER, IR, and $i^{2} E R$ modes over non-cooperative NR mode.

\section{A. Feasibility of Energy Relaying (ER) Mode}

First we derive conditions for improved performance of ER over NR. ER mode is useful when the transmit power $P_{t_{\mathcal{S}}}$ of $\mathcal{S}$, based on its harvested energy $E_{h_{\mathcal{S}_{\text {tot }}}^{\mathrm{ER}}}^{\text {from } \mathcal{A} \text { and } \mathcal{R}}$ jointly, is more than the harvested energy $E_{h_{S}}^{\text {noER }}$ from $\mathcal{A}$ alone, i.e., without ER. Knowing $E_{h_{\mathcal{S}}}^{\mathrm{noER}}=\overline{\mathcal{P}_{h_{\mathcal{A S}}}} N_{s}$ and $\overline{\mathcal{P}_{h_{\mathcal{A S}}}} \approx$ $\mathcal{L}\left(\mu_{\mathcal{P}_{\mathcal{A}}}\right), E_{h_{\mathcal{S}_{\mathrm{tot}}}}^{\mathrm{ER}}$ defined in Section III-C can be rewritten as:

$$
\begin{aligned}
E_{h_{\mathcal{S}_{\mathrm{tot}}}^{\mathrm{ER}}=} & E_{h_{\mathcal{S}}}^{N_{s}-1}+\overline{\mathcal{P}_{h_{\mathcal{S}}}^{2 h o p}}=\overline{\mathcal{P}_{h_{\mathcal{A S}}}}\left(N_{s}-1\right)+ \\
& \mathcal{L}\left(\mu_{\mathcal{P}_{\mathcal{A S}}}+\beta \alpha \mu_{\mathcal{P}_{\mathcal{R S}}}+\mu_{0} \sqrt{\beta \alpha \mu_{\mathcal{P}_{\mathcal{A}}} \mu_{\mathcal{P}_{\mathcal{R S}}}}\right),
\end{aligned}
$$

where $\mu_{0} \triangleq \cos \left(\frac{2 \pi\left(d_{\mathcal{A S}}-d_{\mathcal{R S}}\right)}{\lambda}\right)\left[(K+1) \mathbf{I}_{\mathbf{0}}\left(\frac{K}{2}\right)+K \mathbf{I}_{\mathbf{1}}\left(\frac{K}{2}\right)\right]^{2}$ $\times \frac{\pi \mathrm{e}^{-K-\overline{\psi^{2}}}}{2(K+1)}$. Using (26) and discussion in Sections III-C and IV-B, $P_{t_{\mathcal{S}}}$ using harvested energy $E_{h_{\mathcal{S}_{\mathrm{tot}}}^{\mathrm{ER}}}^{\mathrm{over}} N_{s}$ slots is:

$$
\begin{aligned}
& P_{t_{\mathcal{S}}}=E_{h_{\mathcal{L}_{\mathrm{tot}}}^{\mathrm{ER}}}^{\mathrm{E}}-P_{\mathrm{con}}^{\mathrm{tx}}=\overline{\mathcal{P}_{h_{\mathcal{A S}}}}\left(N_{s}-1\right)+\mathcal{M}_{j}\left(\mu_{\mathcal{P}_{\mathcal{A S}}}+\beta \alpha \mu_{\mathcal{P}_{\mathcal{R S}}}\right. \\
& \left.+\mu_{0} \sqrt{\beta \alpha \mu_{\mathcal{P}_{\mathcal{A S}}} \mu_{\mathcal{P}_{\mathcal{R S}}}}\right)+\mathcal{C}_{j}-P_{\mathrm{con}}^{\mathrm{tx}}, \forall j \in\{(1 \leq j \leq N) \wedge \\
& \left.\left(\mathcal{P}_{\mathrm{th}_{j}} \leq \mu_{\mathcal{P}_{\mathcal{A S}}}+\beta \alpha \mu_{\mathcal{P}_{\mathcal{R S}}}+\mu_{0} \sqrt{\beta \alpha \mu_{\mathcal{P}_{\mathcal{A}}} \mu_{\mathcal{P}_{\mathcal{R} S}}} \leq \mathcal{P}_{\mathrm{th}_{j+1}}\right)\right\} .(27)
\end{aligned}
$$

Next we present an important result on utility of ER mode based on the variation of $\alpha$.
Lemma 1: With $E_{h_{\mathcal{S}_{\mathrm{tot}}}^{\mathrm{ER}}}^{\mathrm{E}}>P_{\mathrm{con}}^{\mathrm{tx}}$, the transmit power $P_{t_{\mathcal{S}}}$ of $\mathcal{S}$ based on its harvested energy $E_{h_{\mathcal{S}_{\text {tot }}}^{\mathrm{ER}}}$ via RF-ET from $\mathcal{A}$ and ER from $\mathcal{R}$ is either: (i) concave increasing function of $\alpha$ when energy signals received at $\mathcal{S}$ in the $N_{s}$ th slot from $\mathcal{A}$ and $\mathcal{R}$ add up constructively, or (ii) strictly-convex in $\alpha$ when energy signals from $\mathcal{A}$ and $\mathcal{R}$ lead to destructive interference.

Proof: First of all we note that, the value of $\mu_{0}$ containing cosine term $\cos (\cdot)$ represents constructive or destructive interference of energy signals from $\mathcal{A}$ and $\mathcal{R}$. $\mu_{0}>0$ always leads to constructive interference, i.e., $E_{h_{S_{\text {tot }}}^{\mathrm{ER}}}>E_{h_{\mathcal{S}_{\mathrm{t}}}}^{\mathrm{noER}}$. However if $\mu_{0}<0$, then received energy signals at $\mathcal{S}$ in $N_{s}$ th slot may add up destructively to cause $E_{h_{\mathcal{S}_{\text {tot }}}^{\mathrm{ER}}}^{\mathrm{ER}} \leq E_{h_{\mathcal{S}_{\mathrm{tot}}}}^{\mathrm{noER}}$.

As $\frac{\partial^{2} P_{t_{S}}}{\partial \alpha^{2}}=-\frac{\mu_{0} \mathcal{M}_{j} \sqrt{\alpha \beta \mu_{\mathcal{P}_{\mathcal{A S}}} \mu_{\mathcal{P}_{\mathcal{R}} \mathcal{S}}}}{4 \alpha^{2}}$, we can observe that $P_{t_{\mathcal{S}}}$ is concave in $\alpha$ if $\mu_{0} \geq 0$; otherwise it is a convex function of $\alpha \forall \mu_{0}<0$. We also note that, since $\frac{\partial P_{t_{\mathcal{S}}}}{\partial \alpha}=\frac{1}{2} \beta \mu_{\mathcal{P}_{\mathcal{R S}}} \mathcal{M}_{j}\left(\frac{\mu_{0} \mu_{\mathcal{P}_{\mathcal{A S}}}}{\sqrt{\alpha \beta \mu_{\mathcal{P}_{\mathcal{A S}}} \mu_{\mathcal{P}_{\mathcal{R}}}}}+2\right), P_{t_{\mathcal{S}}}$ is strictly increasing function of $\alpha \forall \mu_{0} \geq 0 \wedge \beta=1$. On other hand, if $\mu_{0}<0$, a unique feasible critical point $\alpha_{g_{\mathrm{ER}}}=$ $\left\{\alpha \mid\left(\frac{\partial P_{t_{\mathcal{S}}}}{\partial \alpha}=0\right) \wedge(0 \leq \alpha \leq 1)\right\}$ is defined as: $\alpha_{g_{\mathrm{ERR}}} \triangleq$ $\frac{\mu_{0}^{2} \mu_{\mathcal{P}_{\mathcal{A}} \mathcal{S}}}{4 \beta \mu_{\mathcal{P}_{\mathcal{R}}}}$. Thus, for $\mu_{0}<0$ if $\alpha \leq \alpha_{g_{\mathrm{ER}}}$, then $P_{t_{\mathcal{S}}}$ is a decreasing function of $\alpha$. However when $4 \alpha_{g_{\mathrm{ER}}}<\alpha \leq 1$, $P_{t_{\mathcal{S}}}$ is an increasing function of $\alpha$ and even for $\mu_{0}<0$, $\mathcal{L}\left(\mu_{\mathcal{P}_{\mathcal{A S}}}+\beta \alpha \mu_{\mathcal{P}_{\mathcal{R S}}}+\mu_{0} \sqrt{\beta \alpha \mu_{\mathcal{P}_{\mathcal{A S}}} \mu_{\mathcal{P}_{\mathcal{R S}}}}\right)>{\overline{\mathcal{P}_{h}}}_{h_{\mathcal{A}}}$, which leads to the improved ER performance over NR.

Remark 1: Though $\mu_{0}<0$ leads to destructive interference of signals from $\mathcal{A}$ and $\mathcal{R}$, i.e., $\mathcal{L}\left(\mu_{\mathcal{P}_{\mathcal{A S}}}+\beta \alpha \mu_{\mathcal{P}_{\mathcal{R S}}}\right) \geq \mathcal{L}\left(\mu_{\mathcal{P}_{\mathcal{A S}}}\right.$

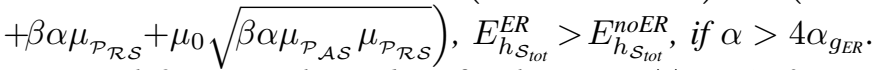

Remark 2: ER is always beneficial over $N R \forall \alpha>0$ if $\mu_{0} \geq$ 0 and $\forall \alpha>4 \alpha_{g_{E R}}$ if $\mu_{0}<0$.

Hence, we conclude that ER is preferred over NR when energy signals received at $\mathcal{S}$ from $\mathcal{A}$ and $\mathcal{R}$ are combined constructively. The chances of ER performing better than NR increase with increasing $P_{t_{\mathcal{R}}}$ which leads to a higher $\mu_{\mathcal{P}_{\mathcal{R S}}}$ because it helps in meeting the condition $\alpha>4 \alpha_{g_{\mathrm{ER}}}$.

\section{B. Feasibility of Information Relaying (IR) Mode}

Now we obtain the feasibility conditions for improved performance of IR over NR mode.

Lemma 2: The normalized delay-limited throughput $\bar{\tau}$ in IR is more than NR if and only if one of these two conditions hold: (i) $p_{\text {out }}^{\mathrm{IR}} \leq p_{\text {out }}^{\mathrm{noIR}}$ or (ii) $p_{\text {out }}^{\mathrm{nolR}}<p_{\text {out }}^{\mathrm{IR}}<\frac{\left(N_{s}+2\right) p_{\text {out }}^{\text {nol }}+N_{s}}{2\left(N_{s}+1\right)}$.

Proof: Firstly, $\frac{\overline{T_{\mathrm{NR}}}}{\overline{\tau_{\mathrm{R}}}}=\frac{\left(N_{s}+2\right)\left(1-p_{\text {out }}^{\text {nolR }}\right)}{2\left(N_{s}+1\right)\left(1-p_{\text {out }}^{\mathrm{R}}\right)}$ and $\frac{1}{2}<\frac{N_{s}+2}{2\left(N_{s}+1\right)}<$ $1, \forall N_{s}>0$. For $\overline{\tau_{\mathrm{IR}}}>\overline{\tau_{\mathrm{NR}}}$, we require $p_{\text {out }}^{\text {nol }}>p_{\text {out }}^{\mathrm{IR}}-\frac{N_{s}\left(1-p_{\text {out }}^{\mathrm{IR}}\right)}{N_{s}+2}$, which is true $\forall p_{\text {out }}^{\mathrm{IR}} \leq p_{\text {out }}^{\mathrm{noIR}}$. Thus, for $\overline{\tau_{\mathrm{IR}}}>\overline{\tau_{\mathrm{NR}}}$, either $p_{\text {out }}^{\mathrm{IR}} \leq$ $p_{\text {out }}^{\text {nolR }}$ or $p_{\text {out }}^{\text {nolR }}<p_{\text {out }}^{\text {IR }}<\frac{\left(N_{s}+2\right) p_{\text {out }}+N_{s}}{2\left(N_{s}+1\right)}$. As $p_{\text {out }}^{\text {nolR }}=\operatorname{Pr}\left(\gamma_{\mathcal{S A}}<\right.$ $\left.2^{R_{0}}-1\right)$ and $p_{\text {out }}^{\mathrm{IR}}=\operatorname{Pr}\left(\min \left\{\gamma_{\mathcal{S R}}, \gamma_{\mathcal{R}_{\mathcal{A}}}+\gamma_{\mathcal{S A}}\right\}<2^{2 R_{0}}-1\right)$, it is worth noting that the outage threshold of $2 R_{0}$ for IR is two times the outage threshold $R_{0}$ for NR.

Following this result, we next discuss the conditions where outage probability $p_{\text {out }}^{\mathrm{IR}}$ in IR and $\mathrm{i}^{2} \mathrm{ER}$ modes is better than outage probability $p_{\text {out }}^{\text {nolr }}$ in no IR modes, namely NR and ER. 
Lemma 3: To ensure that $p_{\text {out }}^{\mathrm{IR}}<p_{\text {out }}^{\text {noIR }}$, the following average SNR conditions should be met: (i) $\mathbb{E}\left[\gamma_{\mathcal{S R}}\right]>\mathbb{E}\left[\gamma_{\mathcal{S A}}\right]\left(\mathbb{E}\left[\gamma_{\mathcal{S A}}\right]\right.$ $+2)$ and (ii) $\mathbb{E}\left[\gamma_{\mathcal{R}_{\mathcal{A}}}\right]>\mathbb{E}\left[\gamma_{\mathcal{S A}}\right]\left(1+\mathbb{E}\left[\gamma_{\mathcal{S A}}\right]\right)$.

Proof: Please refer to Appendix A

Remark 3: With $\mathbb{E}\left[\gamma_{\mathcal{S A}}\right]>0$, Lemma 3 implies that for feasibility of IR, $\mathbb{E}\left[\gamma_{\mathcal{S R}}\right]>\mathbb{E}\left[\gamma_{\mathcal{R A}}\right]$. Or, IR is feasible when $\mathcal{R}$ is placed relatively closer to $\mathcal{S}$ to strengthen $\mathcal{S}$-to- $\mathcal{R}$ link.

Corollary 1: Outage performance for IR is better than NR if relay placement $(\mathrm{RP})\left(d_{\mathcal{S R}}, d_{\mathcal{R} \mathcal{A}}\right)$ lies in the set $\mathbb{S}_{\mathrm{IR}} \triangleq$ $\left\{\left(d_{\mathcal{S R}}, d_{\mathcal{R} \mathcal{A}}\right) \mid\left(d_{\mathcal{S R}}<d_{\mathcal{S A}} \frac{\frac{G_{\mathcal{R}}}{G_{\mathcal{D}}}}{\left(2+\mathbb{E}\left[\gamma_{\mathcal{S} \mathcal{A}}\right]\right)^{\frac{1}{n}}}\right) \wedge\left(d_{\mathcal{R A}}<d_{\mathcal{R} \mathcal{A}}^{\max }\right)\right\}$.

Proof: From Lemma 3, we note that to ensure $p_{\text {out }}^{\mathrm{IR}}<p_{\text {out }}^{\text {noIR }}$, two average SNR conditions (i) and (ii) should be met. Condition (i) on simplification results in the following relationship between $\mathcal{S}$-to- $\mathcal{R}$ and $\mathcal{S}$-to- $\mathcal{A}$ distances: $d_{\mathcal{S R}}<\frac{d_{\mathcal{S A}} \frac{G_{\mathcal{R}}}{G_{\mathcal{D}}}}{\left(2+\frac{\left(N_{s} \overline{\mathcal{P}_{h} \mathcal{A S}}-P_{\mathrm{con}}^{\mathrm{tx}}\right) G_{\mathcal{A}} G_{\mathcal{S}} \lambda^{2}}{(4 \pi \sigma)^{2}\left(d_{\mathcal{S} \mathcal{A}}\right)^{n}}\right)^{\frac{1}{n}}}=d_{\mathcal{S A} \mathcal{A}} \frac{\frac{G_{\mathcal{R}}}{G_{\mathcal{D}}}}{\left(2+\mathbb{E}\left[\gamma_{\mathcal{S A}}\right]\right)^{\frac{1}{n}}}$. Similarly, condition (ii) puts an upper bound $d_{\mathcal{R} \mathcal{A}}^{\max }$ on $\mathcal{R}$-to- $\mathcal{A}$ distance $d_{\mathcal{R A}}$ to meet the $\mathrm{EH}$ requirements of $\mathcal{R}$ for efficient IR, which is given by: $d_{\mathcal{R A}}<d_{\mathcal{R A}_{\mathcal{A}}}^{\max \triangleq}$ $\left\{d_{\mathcal{R} \mathcal{A}} \mid \frac{16 \pi^{2} \mathbb{E}\left[\gamma_{\mathcal{S} \mathcal{A}}\right]\left(1+\mathbb{E}\left[\gamma_{\mathcal{S} \mathcal{A}}\right]\right) \sigma^{2}\left(d_{\mathcal{R} \mathcal{A}}\right)^{n}}{\beta G_{\mathcal{A}} G_{\mathcal{R}} \lambda^{2}\left(\overline{\mathcal{P}}_{h_{\mathcal{A R}}} N_{s}(1-\alpha)+E_{i_{\mathcal{R}}}-P_{\mathrm{con}}^{\mathrm{tx}}-E_{\mathrm{con}}^{\mathrm{rx}}-R_{0} E_{\mathrm{bit}}^{\mathrm{rx}}\right)}=1\right\}$. These bounds on $d_{\mathcal{S R}}$ and $d_{\mathcal{R A}}$ form the feasible RP set $\mathbb{S}_{\mathrm{IR}}$ for enhanced outage performance of IR over NR.

\section{Insights on Optimal Mode Selection Policy}

Using the observations in Sections V-A and V-B, now we provide insights on the mode to be selected among NR, ER, IR, and $\mathrm{i}^{2}$ ER based on the two decision making (cf. Fig. 2) for minimizing outage probability. From Lemma 2, improved outage performance in IR or $\mathrm{i}^{2} \mathrm{ER}$ mode also implies that their throughput performance is better than ER or NR mode.

1) Decision I: IR or no IR?: From Lemma 3 and Corollary 1 , we note that if relay placement $\left(d_{\mathcal{S R}}, d_{\mathcal{R A}}\right) \in \mathbb{S}_{\mathrm{IR}}$, then the outage probability $p_{\text {out }}^{\mathrm{IR}}$ in two-hop IT in IR and $\mathrm{i}^{2} \mathrm{ER}$ modes is better or lower than the outage probability $p_{\text {out }}^{\text {noIR }}$ in single-hop IT in ER and NR modes. So with the available statistical CSI, decoding capability of $\mathcal{R}$ based on its harvested energy and $\mathcal{S}$-to- $\mathcal{R}$ link quality is decided. Only when decoding capability is sufficiently large such that either of IR or $\mathrm{i}^{2} \mathrm{ER}$ modes perform better than ER or NR modes, $\mathcal{R}$ invests its harvested energy on IR. Otherwise, it utilizes its energy for ER or saves it for future if a NR is chosen.

Further, as $\mathrm{i}^{2} \mathrm{ER}$ with $\alpha=0$ reduces to IR mode, the feasibility conditions for $\mathrm{i}^{2} \mathrm{ER}$ mode are similar to as in IR mode, which are mentioned in Lemma 3. However, when both IR and $\mathrm{i}^{2} \mathrm{ER}$ are feasible, i.e., $p_{\text {out }}^{\mathrm{IR}}<p_{\text {out }}^{\text {noIR }}$ for $\alpha=0$, then $\mathrm{i}^{2} \mathrm{ER}$ can provide better performance than IR by allowing integrated IR and ER, as discussed next.

2) Decision II: ER or no ER?: The decision for ER depends on whether the received energy signals from $\mathcal{A}$ and $\mathcal{R}$ add constructively or destructively. The conditions for preferring ER over NR mode based on the value of $\alpha$ and $\mu_{0}$ have been presented in Lemma 1 and Remark 2.

When IR mode is feasible, then for $\mu_{0} \geq 0, \mathrm{i}^{2} \mathrm{ER}$ can provide better outage performance if $\frac{\partial \mathbb{E}\left[\gamma_{\mathcal{R A}}+\gamma_{\mathcal{S A}}\right]}{\partial \alpha} \geq 0$. In other words, if both $\mathbb{E}\left[\gamma_{\mathcal{S R}}\right]$ and $\mathbb{E}\left[\gamma_{\mathcal{R A}}+\gamma_{\mathcal{S A}}\right]$ are increasing in $\alpha$, then from Theorem 1 (Section VI-B), $p_{\text {out }}^{\mathrm{IR}}$ in $\mathrm{i}^{2} \mathrm{ER}$ is a decreasing function of $\alpha$ implying that its outage performance with $\alpha>0$ is better than that of IR mode having $\alpha=0$.

\section{Optimal Sharing of Harvested EnERGy at $\mathcal{R}$}

Following the observations in previous section, now we optimize $\alpha$ to efficiently utilize the available harvested energy at $\mathcal{R}$ for ER and IR. First we formulate the optimization problem, followed by its generalized-convexity proof and the joint global-optimal solution $\left(R_{0}^{*}, \alpha^{*}, \beta^{*}\right)$.

\section{A. Optimization Formulation}

We intend to maximize the normalized throughput $\bar{\tau}$ by efficiently dividing harvested energy at $\mathcal{R}$ over $N_{s}$ slots, i.e., $\alpha$ fraction for ER and remaining $(1-\alpha)$ fraction for IR. As $\bar{\tau}$ defined in (25) is a function of rate constraint $R_{0} \mathrm{bps} / \mathrm{Hz}$ to be met at $\mathcal{A}$ during the IT phase of 1 or 2 slots, we also find maximum achievable rate $R_{0}$ that can be met with high probability $1-p_{\text {out }}^{t h}$, where $p_{\text {out }}^{t h} \ll 1$. This is denoted by constraint $C 1$ in throughput maximization problem (P1).

$$
\begin{array}{r}
\text { (P1): } \operatorname{maximize}_{R_{0}, \alpha, \beta} \bar{\tau}, \quad \text { subject to: } \quad C 1: p_{\text {out }} \leq p_{\text {out }}^{\text {th }}, \\
C 2: \alpha \geq 0, \quad C 3: \alpha \leq 1, \quad C 4: \beta \in\{0,1\} .
\end{array}
$$

Here $\beta=1$ or $\beta=0$ is respectively based on whether to go for relaying (ER, IR, or $\left.\mathrm{i}^{2} \mathrm{ER}\right)$ or not $(\mathrm{NR})$. As $(\mathrm{P} 1)$ is nonconvex, it is difficult to jointly optimize $R_{0}, \alpha$, and $\beta$. So, we break the problem (P1) into two parts, i.e., first solve outage minimization problem $(\mathrm{P} 2)$ to find optimal $\alpha$ that minimizes $p_{\text {out }}^{\mathrm{IR}}$. After that we use monotonicity of $p_{\text {out }}$ in $R_{0}$ to iteratively solve (P1).

(P2) : $\underset{\alpha}{\operatorname{minimize}} p_{\text {out }}^{\mathrm{IR}}, \quad$ subject to: $C 2$ and $C 3$.

Remark 4: Using the statistical CSI along with the system parameters mentioned in Sections II, III, and IV, energy-rich $\mathcal{A}$ solves (P1) and informs $\mathcal{R}$ and $\mathcal{S}$ respectively about the optimal relaying mode $\left(\alpha^{*}, \beta^{*}\right)$ and optimal $R_{0}^{*}$ to maximize the normalized delay-limited throughput.

\section{B. Generalized-Convexity of Outage Minimization Problem}

Here we present some important results in the form of Lemma 4, Corollary 2, and Theorem 1, that will be useful in proving conditional generalized-convexity [33] of (P2).

Lemma 4: The average SNR $\mathbb{E}\left[\gamma_{\mathcal{R} \mathcal{A}}+\gamma_{\mathcal{S A}}\right]$ for $\mathcal{S}-\mathcal{R}-\mathcal{A}$ link is: (a) strictly concave in $\alpha$ if $\mu_{0}>0$ and (b) convex function of $\alpha$ for $\mu_{0} \leq 0$ with unique stationary point denoted by $\alpha_{g_{\mathrm{IR}}}$.

Proof: Using linearity of expectation in $\mathbb{E}\left[\gamma_{\mathcal{R A}}+\gamma_{\mathcal{S A}}\right]$,

$$
\begin{aligned}
& \mathbb{E}\left[\gamma_{\mathcal{R} \mathcal{A}}\right.\left.+\gamma_{\mathcal{S A}}\right]=\mathbb{E}\left[\gamma_{\mathcal{R} \mathcal{A}}\right]+\mathbb{E}\left[\gamma_{\mathcal{S} \mathcal{A}}\right]=\frac{\mu_{\mathcal{P}_{\mathcal{S R}}}}{\sigma^{2}}+\frac{\mu_{\mathcal{P}_{\mathcal{S} \mathcal{A}}}}{\sigma^{2}} \\
&=\left(\frac{(1-\alpha) \overline{\mathcal{P}_{h_{\mathcal{A R}}}} N_{s}+E_{i_{\mathcal{R}}}-P_{\mathrm{con}}^{\mathrm{tx}}-E_{\mathrm{con}}^{\mathrm{rx}}-R_{0} E_{\mathrm{bit}}^{\mathrm{rx}}}{\left(\beta G_{\mathcal{R}}\right)^{-1}\left(d_{\mathcal{R} \mathcal{A}}\right)^{n}}\right. \\
&\left.+\frac{\overline{\mathcal{P}_{h_{\mathcal{A}}}}\left(N_{s}-1\right)+\mathcal{M}_{j} \overline{\mathcal{P}_{h_{\mathcal{S}}}^{2 \mathrm{hop}}}+\mathcal{C}_{j}-P_{\mathrm{con}}^{\mathrm{tx}}}{\left(G_{\mathcal{S}}\right)^{-1}\left(d_{\mathcal{S A}}\right)^{n}}\right) \frac{G_{\mathcal{A}}}{\sigma^{2}}\left(\frac{\lambda}{4 \pi}\right)^{2}, \\
& \forall j \in\left\{(1 \leq j \leq N) \wedge\left(\mathcal{P}_{\mathrm{th}_{j}} \leq \overline{\mathcal{P}_{h_{\mathcal{S}}}^{2 \mathrm{hop}}} \leq \mathcal{P}_{\mathrm{th}_{j+1}}\right)\right\},
\end{aligned}
$$


where $\overline{\mathcal{P}_{h_{\mathcal{S}}}^{\text {2hop }}}=\mu_{\mathcal{P}_{\mathcal{A S}}}+\beta \alpha \mu_{\mathcal{P}_{\mathcal{R} \mathcal{S}}}+\mu_{0} \sqrt{\beta \alpha \mu_{\mathcal{P}_{\mathcal{A S}}} \mu_{\mathcal{P}_{\mathcal{R} \mathcal{S}}}}$. From $\frac{\partial^{2} \mathbb{E}\left[\gamma_{\mathcal{R} \mathcal{A}}+\gamma_{\mathcal{S} \mathcal{A}}\right]}{\partial \alpha^{2}}=-\frac{G_{\mathcal{S}} G_{\mathcal{A}} \lambda^{2} \mu_{0} \mathcal{M}_{j} \sqrt{\alpha \beta \mu_{\mathcal{P}_{\mathcal{S}}} \mu_{\mathcal{P}_{\mathcal{R} \mathcal{S}}}}}{4(4 \pi)^{2} \sigma^{2} \alpha^{2}\left(d_{\mathcal{S} \mathcal{A}}\right)^{n}}$, we note that depending on whether $\mu_{0}>0$ or $\mu_{0} \leq 0, \mathbb{E}\left[\gamma_{\mathcal{R A}}+\gamma_{\mathcal{S A}}\right]$ is respectively strictly concave or convex in $\alpha$. Further, it may

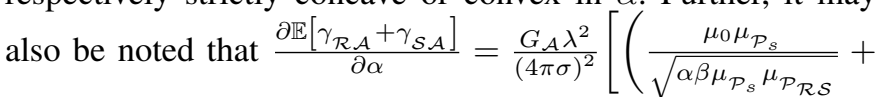
2) $\left.\frac{\beta G_{\mathcal{S}} \mu_{\mathcal{P}_{\mathcal{R S}}} \mathcal{M}_{j}}{2\left(d_{\mathcal{S A}}\right)^{n}}-\frac{\beta G_{\mathcal{R}} N_{s} \overline{\mathcal{P}_{h} \mathcal{A R}}}{\left(d_{\mathcal{R A}}\right)^{n}}\right]$, using which the unique gradient point $\alpha_{g_{\mathrm{IR}}}$, satisfying $\frac{\partial \mathbb{E}\left[\gamma_{\mathcal{R A}}+\gamma_{\mathcal{S A}}\right]}{\partial \alpha}=0$, is given by: $\alpha_{g_{\mathrm{IR}}}=\frac{1}{4 \beta}\left[\frac{\left(d_{\mathcal{R} \mathcal{A}}\right)^{n} G_{\mathcal{S}} \mu_{0} \sqrt{\mu_{\mathcal{P}_{\mathcal{A S}}} \mu_{\mathcal{P}_{\mathcal{R S}}} \mathcal{M}_{j}}}{\left(d_{\mathcal{S A}}\right)^{n} G_{\mathcal{R}} N_{s} \overline{\mathcal{P}_{h_{\mathcal{A R}}}}-\left(d_{\mathcal{R}_{\mathcal{A}}}\right)^{n} G_{\mathcal{S}} \mu_{\mathcal{P}_{\mathcal{R} S}} \mathcal{M}_{j}}\right]^{2}$. So for $\mu_{0} \leq 0, \mathbb{E}\left[\gamma_{\mathcal{R A}}+\gamma_{\mathcal{S A}}\right]$ is strictly decreasing in $\alpha \in\left(0, \alpha_{g_{\mathrm{IR}}}\right)$ and strictly increasing in $\alpha \in\left(\alpha_{g_{\mathrm{IR}}}, 1\right)$.

Corollary 2: The average SNR $\mathbb{E}\left[\gamma_{\mathcal{S R}}\right]$ for $\mathcal{S}$-to- $\mathcal{R}$ link is concave increasing in $\alpha$ if $\mu_{0}>0$, and strictly-convex function of $\alpha$ for $\mu_{0} \leq 0$ with unique critical point $\alpha_{g_{\mathrm{ER}}}$, if it exists.

Proof: As defined in Section IV-B, the average SNR $\mathbb{E}\left[\gamma_{\mathcal{S R}}\right]$ for $\mathcal{S}$-to- $\mathcal{R}$ link is given by:

$$
\mathbb{E}\left[\gamma_{\mathcal{S R}}\right]=\frac{\mu_{\mathcal{P}_{\mathcal{S R}}}}{\sigma^{2}}=\frac{P_{t_{\mathcal{S}}} G_{\mathcal{S}} G_{\mathcal{R}}}{\sigma^{2}\left(d_{\mathcal{R S}}\right)^{n}}\left(\frac{\lambda}{4 \pi}\right)^{2}
$$

where $P_{t_{\mathcal{S}}}$ is defined in (27). As $\mathbb{E}\left[\gamma_{\mathcal{S R}}\right]$ is positive affine function of $P_{t_{\mathcal{S}}}$, from Lemma 1 we observe that $\mathbb{E}\left[\gamma_{\mathcal{S} \mathcal{R}}\right]$ is respectively concave increasing and strictly-convex in $\alpha$ for $\mu_{0}>0$ and $\mu_{0} \leq 0$. The unique critical point $\alpha_{g_{\mathrm{ER}}}=\frac{\mu_{0}^{2} \mu_{\mathcal{P}_{\mathcal{A S}}}}{4 \beta \mu_{\mathcal{P}_{\mathcal{R S}}}}$ for $\mu_{0} \leq 0$, if exists, is defined in Lemma 1 .

Theorem 1: The complimentary CDFs (CCDFs) $\overline{F_{\mathcal{P}_{r_{\mathcal{S R}}}}}$ and $\overline{F_{\mathcal{P}_{r_{\mathcal{A}}}+\mathcal{P}_{r_{\mathcal{S A}}}}}$ of received powers $\mathcal{P}_{\mathcal{S R}}$ and $\mathcal{P}_{\mathcal{R A}}+\mathcal{P}_{\mathcal{S A}}$ are respectively positive increasing log-concave functions of $\mathbb{E}\left[\gamma_{\mathcal{S R}}\right]$ and $\mathbb{E}\left[\gamma_{\mathcal{R A}}+\gamma_{\mathcal{S A}}\right]$

Proof: Please refer to Appendix B.

By using these results, the conditional-pseudoconvexity and global-optimality of (P2) are discussed next.

Theorem 2: As the objective function of (P2) is pseudoconvex in $\alpha \in\left\{\begin{array}{ll}{[0,1],} & \mu_{0}>0 \\ {\left[\alpha_{g_{\mathrm{ER}}}, \alpha_{g_{\mathrm{IR}}}\right],} & \mu_{0} \leq 0,\end{array}\right.$ and constraints $C 2, C 3$ are affine functions of $\alpha$, there exists a unique global-optimal solution $\alpha^{*} \in\left[\alpha_{g_{\mathrm{ER}}}, \alpha_{g_{\mathrm{IR}}}\right]$ that minimizes $p_{\text {out }}^{\mathrm{IR}}$.

Proof: Please refer to Appendix C.

\section{Global Optimal Allocation of Harvested Energy at Relay}

Theorem 3: The global optimal utilization of harvested energy at $\mathcal{R}$ for minimizing $p_{\text {out }}^{\mathrm{IR}}$ is given by $\alpha^{*} \triangleq$

$$
\begin{gathered}
\underset{\alpha=\left\{0, \alpha_{\text {out }}, 1\right\}}{\operatorname{argmin}}\left\{\left.p_{\text {out }}^{\mathrm{IR}}\right|_{\alpha=0},\left.p_{\text {out }}^{\mathrm{IR}}\right|_{\alpha=\alpha_{\text {out }}},\left.p_{\text {out }}^{\text {noIR }}\right|_{\alpha=1}\right\} \text {, with } \\
\alpha_{\text {out }} \triangleq \begin{cases}\alpha_{\min }^{\text {out }}, & \alpha_{\text {cri }}^{\text {out }}<\alpha_{\min }^{\text {out }}, \\
\alpha_{\text {cri }}^{\text {out }}, & \alpha_{\text {min }}^{\text {out }} \leq \alpha_{\text {cri }}^{\text {out }} \leq \alpha_{\max }^{\text {out }}, \\
\alpha_{\text {max }}^{\text {out }}, & \text { otherwise. }\end{cases}
\end{gathered}
$$

Here $\alpha_{\text {min }}^{\text {out }} \triangleq \max \left\{\frac{z+\mu_{0}^{2} \mu_{\mathcal{P}_{\mathcal{A S}}} \mathcal{M}_{j}+\sqrt{\mu_{0}^{2} \mu_{\mathcal{P}_{\mathcal{A S}}}\left(2 z+\mu_{0}^{2} \mu_{\mathcal{P}_{\mathcal{A S}}} \mathcal{M}_{j}\right)}}{2 \beta \mu_{\mathcal{P}_{\mathcal{R S}} \mathcal{M}_{j}}}, 0\right.$, $\left.\alpha_{g_{\mathrm{ER}}}\right\}, \alpha_{\text {cri }}^{\text {out }} \triangleq\left\{\alpha \mid\left(\frac{\partial p_{\text {out }}^{\mathrm{IR}}}{\partial \alpha}=0\right) \wedge(0<\alpha<1)\right\}, \alpha_{\max }^{\text {out }} \triangleq \min \{1$,
$\left.\alpha_{g_{\mathrm{IR}}}, 1-\frac{E_{\text {bit }}^{\mathrm{rx}} R_{0}+E_{\mathrm{con}}^{\mathrm{rx}}+P_{\mathrm{con}}^{\mathrm{tx}}-E_{i_{\mathcal{R}}}}{N_{s} \overline{\mathcal{P}}_{h_{\mathcal{A R}}}}\right\}$, and $z \triangleq-2\left(\mathcal{C}_{j}+\mu_{\mathcal{P}_{\mathcal{A S}}} \mathcal{M}_{j}+\right.$ $\left.\left(N_{s}-1\right) \overline{\mathcal{P}_{h_{\mathcal{A S}}}}-P_{\text {con }}^{\mathrm{tx}}\right)+\mu_{0}^{2} \mu_{\mathcal{P}_{\mathcal{A S}}} \mathcal{M}_{j}$ with $\overline{\mathcal{P}_{h_{\mathcal{S}}}^{2 \mathrm{hop}}} \in\left[\mathcal{P}_{\mathrm{th}_{j}}, \mathcal{P}_{\mathrm{th}_{j+1}}\right]$. Proof: As $p_{\text {out }}^{\mathrm{IR}}$ is pseudoconvex or unimodal ${ }^{1}$ in $\alpha \in$ $\left[\alpha_{g_{\mathrm{ER}}}, \alpha_{g_{\mathrm{IR}}}\right] \forall \mu_{0}$ (cf. Theorem 2), the global-optimal solution $\alpha^{*}$ is given by the unique mode $\alpha_{c r i}^{\text {out }}$, defined in (C.3) by solving $\frac{\partial p_{\text {out }}^{\mathrm{IR}}}{\partial \alpha}=0$, if it exists in the feasible region defined by $C 2-C 3$ for $\mu_{0}>0$ or satisfies condition $\alpha_{c r i}^{\text {out }} \in$ $\left[\alpha_{g_{\mathrm{ER}}}, \alpha_{g_{\mathrm{IR}}}\right], \forall \mu_{0} \leq 0$. However if $\alpha_{\text {cri }}^{\text {out }}>\alpha_{\max }^{\text {out }}$ or $\alpha_{c r i}^{\text {out }}<$ $\alpha_{\text {min }}^{\text {out }}$, then due to the corresponding monotonically decreasing or increasing trend of $p_{\text {out }}^{\mathrm{IR}}$ with $\alpha$, global-optimal $\alpha^{*}$ is given by the two corner points $\alpha_{\max }^{\text {out }}$ and $\alpha_{\min }^{\text {out }}$, respectively. $\alpha_{\max }^{\text {out }}$ ensures that $C 3$ is satisfied, $\alpha_{\text {out }} \leq \alpha_{g_{\mathrm{IR}}} \forall \mu_{0} \leq 0$ and $P_{t_{\mathcal{R}}}>0$. Similarly, $\alpha_{\text {min }}^{\text {out }}$ ensures that $C 2$ is satisfied, $\alpha_{\text {out }} \geq \alpha_{g_{\mathrm{ER}}} \forall \mu_{0} \leq 0$ and $P_{t_{\mathcal{S}}}>0$. As for $\mu_{0} \leq 0, p_{\text {out }}^{\mathrm{IR}}$ respectively follows monotonically increasing and decreasing trend with $\alpha$, for $\alpha<\alpha_{g_{\mathrm{ER}}}$ and $\alpha>\alpha_{g_{\mathrm{IR}}}$, optimal $\alpha^{*}$ is given by one of the three potential candidates, i.e., $\alpha \in\left\{0, \alpha_{\text {out }}, 1\right\}$. Also, it may be noted that for $\mu_{0}>0, \alpha_{\text {out }}=\alpha_{\text {cri }}^{\text {out }}$ itself.

Remark 5: Although $\alpha=1$ is shown as a feasible solution for $(P 2)$ to minimize $p_{\text {out }}^{I R}, \alpha=1$ leads to $P_{t_{\mathcal{R}}}=0$ implying that no communication takes place from $\mathcal{R}$-to- $\mathcal{A}$, i.e. no IR for $\alpha=1$. So if $\alpha^{*}=1$, then $p_{\text {out }}=p_{\text {out }}^{\text {noIR }}$ as given by (24).

\section{Iterative Scheme to Maximize Normalized Throughput $\bar{\tau}$}

Now we try to maximize $\bar{\tau}$ by jointly optimizing $R_{0}, \alpha$, and $\beta$ in problem (P1). Since (P1) is nonconvex and has combinatorial aspect due to inherent mode selection in definition of $\bar{\tau}$ given in (25), we make use of Theorem 3 to find optimal $\alpha^{*}$ for a given $R_{0}$ with $\beta=1$. In this regard we present an iterative scheme, named Algorithm 1, that helps in maximizing $\bar{\tau}$ by iteratively optimizing $\alpha$ and $R_{0}$. Here while optimizing $R_{0}$ to meet certain quality-of-service (QoS) requirement, we need to ensure that corresponding $p_{\text {out }}<p_{\text {out }}^{\text {th }}$ (constraint $C 1$ ).

The iterative scheme starts with finding $R_{0}$ that satisfies $p_{\text {out }}^{\text {noIR }} \leq p_{\text {out }}^{\text {th }}$ for ER and NR modes, denoted by $R_{0}^{\mathrm{ER}}$ and $R_{0}^{\mathrm{NR}}$, respectively. These $R_{0}^{\mathrm{ER}}$ and $R_{0}^{\mathrm{NR}}$ values are obtained by finding inverse of the CDF function of $\mathcal{P}_{r_{\mathcal{S A}}}$ using Algorithm 2 with $\alpha=\beta=1$ and $\alpha=\beta=0$, respectively. With initial $R_{0}$ being $R_{0}^{(0)}=\frac{N_{s}+2}{2\left(N_{s}+1\right)} \max \left\{R_{0}^{\mathrm{ER}}, R_{0}^{\mathrm{NR}}\right\}$, we find optimal $\alpha^{*}$ minimizing $p_{\text {out }}^{\mathrm{IR}}$ by using Theorem 3. If $0<\alpha^{*}<1$ with $\beta^{(1)}=1$, then this implies that neither of NR or ER modes can provide the optimal $R_{0}^{*}$, or in other words, $R_{0}^{*}>R_{0}^{(0)}$. So $\alpha^{*}=\alpha^{(1)}$ with $R_{0}=R_{0}^{(0)}$ results in a $p_{\text {out }}^{*}$ which is lower than $p_{\text {out }}^{\text {th }}$ and the Algorithm 1 continues. This decrease in $p_{\text {out }}^{*}$ implies that we can achieve higher $R_{0}$ due to the improved end-to-end SNR quality. Next we find updated $R_{0}$, denoted by $R_{0}^{(1)}$, satisfying $p_{\text {out }}^{\mathrm{IR}} \leq p_{\text {out }}^{\text {th }}$ for $\mathrm{i}^{2} \mathrm{ER}$ by using inverse of CDF of min $\left\{\mathcal{P}_{r_{\mathcal{S}}}, \mathcal{P}_{r_{\mathcal{R} \mathcal{A}}}+\mathcal{P}_{r_{\mathcal{S A}}}\right\}$ with $\mathbb{E}\left[\mathcal{P}_{r_{\mathcal{S}}}\right]$ and $\mathbb{E}\left[\mathcal{P}_{r_{\mathcal{R A}}}+\mathcal{P}_{r_{\mathcal{S A}}}\right]$ defined using $\alpha=\alpha^{*}$. The iterative process continues till $\left|R_{0}^{(i)}-R_{0}^{(i-1)}\right| \leq \xi_{R_{0}}$, where $\xi_{R_{0}} \ll 1$ is the acceptable tolerance. Algorithm 1 terminates with the optimal $R_{0}^{*}, \alpha^{*}, \beta^{*}$ that provide maximum $\bar{\tau}^{*}$ by selecting the optimal relaying mode and maximum achievable rate $R_{0}^{*}$

${ }^{1}$ It may be noted that unimodality (having unique minima) of a single variable function is equivalent to its pseudoconvexity [34]. 
$\overline{\text { Algorithm } 1 \text { Iterative scheme to maximize normalized throughput }}$ $\bar{\tau}$ by jointly optimizing $R_{0}, \alpha, \beta$

Input: Relay position $\left(d_{\mathcal{R} \mathcal{A}}, d_{\mathcal{S R}}\right)$, system and channel parameters (cf. Section II), with tolerances $p_{\text {out }}^{\text {th }}, \xi_{R_{0}}, \xi$

Output: Maximized throughput $\bar{\tau}^{*}$ along with optimal $R_{0}^{*}, \alpha^{*}, \beta^{*}$ (A) Initialization

1: Call Algorithm 2 to find $\Upsilon_{\mathrm{ER}}=\left\{\Upsilon|| p_{\text {out }}^{\text {th }}-F_{\mathcal{P}_{r_{\mathcal{S A}}}}\left(\Upsilon, K_{\mathcal{S A}}\right.\right.$, $\left.\left.\frac{\mu_{\mathcal{P}_{\mathcal{S} \mathcal{A}}}}{\sigma^{2}}\right) \mid \leq \xi\right\}$ for $\mathcal{S}$-to- $\mathcal{A}$ link in ER mode with $\mathcal{P}_{r}=$ $\mathcal{P}_{r_{\mathcal{S A}}}, \alpha=1, \beta=1$, and $\widehat{p_{\text {out }}}=p_{\text {out }}^{\text {th }}$

2: Call Algorithm 2 to find $\Upsilon_{\mathrm{NR}}=\left\{\Upsilon|| p_{\text {out }}^{\text {th }}-F_{\mathcal{P}_{r_{\mathcal{S A}}}}\left(\Upsilon, K_{\mathcal{S A}}\right.\right.$, $\left.\left.\frac{\mu_{\mathcal{P}_{\mathcal{S} \mathcal{A}}}}{\sigma^{2}}\right) \mid \leq \xi\right\}$ for $\mathcal{S}$-to- $\mathcal{A}$ link in NR mode with $\mathcal{P}_{r}=$ $\mathcal{P}_{r_{\mathcal{S A}}}, \alpha=0, \beta=0$, and $\widehat{p_{\text {out }}}=p_{\text {out }}^{\text {th }}$

3: Set $i \leftarrow 0, \quad R_{0}^{(0)} \leftarrow \frac{N_{s}+2}{2\left(N_{s}+1\right)} \log _{2}\left(1+\max \left\{\Upsilon_{\mathrm{ER}}, \Upsilon_{\mathrm{NR}}\right\}\right)$

(B) Recursion

4: repeat (Main Loop)

5: $\quad$ Set $i \leftarrow i+1, \quad \alpha_{(i)}^{*} \leftarrow \alpha^{*}$ that minimizes $p_{\text {out }}^{\mathrm{IR}}$ for achieving rate $R_{0}^{(i-1)}$ in $\mathrm{i}^{2}$ ER using Theorem 3

6: $\quad$ Call Algorithm 2 to find $\Upsilon_{\text {SRA }}^{(i)}=\left\{\Upsilon|| p_{\text {out }}^{t h}-(1-[1-\right.$

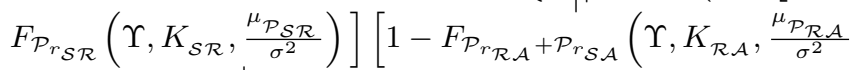
$\left.\left.\left.\left.K_{\mathcal{S A}}, \frac{\mu_{\mathcal{P}_{\mathcal{S} \mathcal{A}}}}{\sigma^{2}}\right)\right]\right) \mid \leq \xi\right\}$ in $\mathrm{i}^{2} \mathrm{ER}$ with $\mathcal{P}_{r}=\min \left\{\mathcal{P}_{r_{\mathcal{S R}}}\right.$, $\left.\mathcal{P}_{r_{\mathcal{R} \mathcal{A}}}+\mathcal{P}_{r_{\mathcal{S} \mathcal{A}}}\right\}, \alpha=\alpha_{(i)}^{*}, \beta=1, \widehat{p_{\text {out }}}=p_{\text {out }}^{\text {th }}$

7: $\quad$ Set $R_{0}^{(i)} \leftarrow \frac{1}{2} \log _{2}\left(1+\Upsilon_{\text {SRA }}^{(i)}\right)$

8: until $\left(\left|R_{0}^{(i)}-R_{0}^{(i-1)}\right| \leq \xi_{R_{0}}\right)$

(C) Termination with Optimal Solution

9: Set $\mathbb{R}_{0,1}=\log _{2}\left(1+\Upsilon_{\mathrm{ER}}\right), \mathbb{R}_{0,2}=\log _{2}\left(1+\Upsilon_{\mathrm{NR}}\right), \quad \mathbb{R}_{0,3}=R_{0}^{(i)}$ 10: Set $j^{*} \leftarrow \underset{1 \leq j \leq 3}{\operatorname{argmax}} \mathbb{R}_{0, j}$, and optimal $\left\{R_{0}^{*}, \alpha^{*}, \beta^{*}\right\}$ is given by $\left\{\begin{array}{lll}\left\{\mathbb{R}_{0,1}, 1,1\right\}, & j^{*}=1 & \text { (ER mode) } \\ \left\{\mathbb{R}_{0,2}, 0,0\right\}, & j^{*}=2 & (\text { NR mode) } \\ \left\{\mathbb{R}_{0,3}, \alpha_{(i)}^{*}, 1\right\}, & j^{*}=3 & \left(\mathrm{i}^{2} \text { ER mode }\left(\mathrm{IR} \text { if } \alpha_{(i)}^{*}=0\right)\right)\end{array}\right.$

with $p_{\text {out }} \leq p_{\text {out }}^{\text {th }}$. Thus, with increasing iteration $(i),\left\{R_{0}^{(i)}\right\}$ monotonically increases (i.e., $R_{0}^{(i+1)}>R_{0}^{(i)}$ ) because of monotonically improving end-to-end SNR due to the optimal relaying mode selection for increasing $\left\{R_{0}^{(i)}\right\}$.

Fast Convergence of Algorithms 1 and 2: Due to strict monotonicity and pseudoconvexity of $p_{\text {out }}$ in $R_{0}$ and $\alpha$ respectively, $\alpha_{(i)}^{*}$ in each iteration can be found efficiently and in general Algorithm 1 converges to acceptable optimal solution $R_{0}^{*}$ in two to three iterations only.

Similarly, Algorithm 2 employing a modified version of Newton-Raphson method, provides fast convergence to the inverse $\Upsilon$ of $\operatorname{CDF} F_{\mathcal{P}_{r}}$, where $\Upsilon$ is defined in steps 1,2 , and 6 of Algorithm 1 for $\mathcal{P}_{r}$ in different relaying modes, due to the following properties: (i) $F_{\mathcal{P}_{r}}$ is monotonically increasing in $\Upsilon$. (ii) $F_{\mathcal{P}_{r}}$ is continuously differentiable positive log-concave in $\Upsilon \in[0, \infty)$. (iii) $\frac{\partial F_{\mathcal{P}_{r}}}{\partial \Upsilon}$ is continuously differentiable logconcave function of $\Upsilon$. (iv) $\mathbb{E}\left[\frac{\mathcal{P}_{r}}{\sigma^{2}}\right]$ provides a very good starting point. We noted that with conventional update equation $\Upsilon^{(i)} \leftarrow \Upsilon^{(i-1)}+\frac{\bar{F}^{(i-1)}-[1-\widehat{\text { out }}]}{f_{\mathcal{P}_{r_{\mathcal{S A}}}}\left(\Upsilon^{(i-1)}, K_{\mathcal{S A}}, \frac{{ }^{\mu} \mathcal{S A}}{\sigma^{2}}\right)}$ in standard NewtonRaphson method, iterations sometimes diverge. To overcome this drawback we consider the usage of log function with which convergence improves significantly. Via extensive nu- $\overline{\text { Algorithm } 2 \text { Iterative scheme to obtain inverse } \Upsilon={F_{\mathcal{P}_{r}}}^{-1}\left(\widehat{p_{\text {out }}}\right)}$ satisfying $\widehat{p_{\text {out }}}=F_{\mathcal{P}_{r}}\left(\Upsilon, K, \mu_{\mathcal{P}}\right)$

Input: $\operatorname{CDF} F_{\mathcal{P}_{r}}, \operatorname{PDF} f_{\mathcal{P}_{r}}$, and mean $\frac{\mu_{\mathcal{P}}}{\sigma^{2}}$ of $\frac{\mathcal{P}_{r}}{\sigma^{2}}$ along with $\alpha, \beta$, $K$, and tolerance $\xi$ for acceptable outage probability $\widehat{p_{\text {out }}}$. Here $\mathcal{P}_{r} \in\left\{\mathcal{P}_{r_{\mathcal{S A}}}, \min \left\{\mathcal{P}_{r_{\mathcal{S}}}, \mathcal{P}_{r_{\mathcal{R} \mathcal{A}}}+\mathcal{P}_{r_{\mathcal{S} \mathcal{A}}}\right\}\right\}$

Output: Inverse $\Upsilon^{*}=\left\{\Upsilon\left|\widehat{p_{\text {out }}}-F_{\mathcal{P}_{r}}\left(\Upsilon, K, \frac{\mu_{\mathcal{P}}}{\sigma^{2}}\right)\right| \leq \xi\right\}$ of $F_{\mathcal{P}_{r}}$

1: Set $i \leftarrow 0$

2: if $\left(\mathcal{P}_{r}=\mathcal{P}_{r_{\mathcal{S A}}}\right)$ then

3: $\quad$ Set $\Upsilon^{(0)} \leftarrow \frac{\mu_{\mathcal{P}_{\mathcal{S A}}}}{\sigma^{2}}, \bar{F}^{(0)} \leftarrow 1-F_{\mathcal{P}_{r_{\mathcal{S A}}}}\left(\Upsilon^{(0)}, K_{\mathcal{S A}}, \frac{\mu_{\mathcal{P}_{\mathcal{S} \mathcal{A}}}}{\sigma^{2}}\right)$

4: else if $\left(\mathcal{P}_{r}=\min \left\{\mathcal{P}_{r_{\mathcal{S} \mathcal{R}}}, \mathcal{P}_{r_{\mathcal{R} \mathcal{A}}}+\mathcal{P}_{r_{\mathcal{S} \mathcal{A}}}\right\}\right)$ then

5: $\quad$ Set $\Upsilon^{(0)} \leftarrow \frac{\min \left\{\mu_{\mathcal{P}_{\mathcal{S R}}}, \mu_{\mathcal{P}_{\mathcal{S A}}}+\mu_{\mathcal{P}_{\mathcal{R} \mathcal{A}}}\right\}}{\sigma^{2}}$

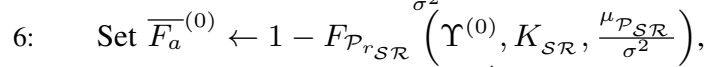

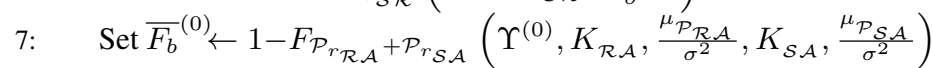

8: $\quad$ Set $\bar{F}^{(0)} \leftarrow{\overline{F_{a}}}^{(0)} \cdot{\overline{F_{b}}}^{(0)}$

9: repeat (Main Loop)

10: $\quad$ Set $i \leftarrow i+1$

11: $\quad$ if $\left(\mathcal{P}_{r}=\mathcal{P}_{r_{\mathcal{S A}}}\right)$ then

12:

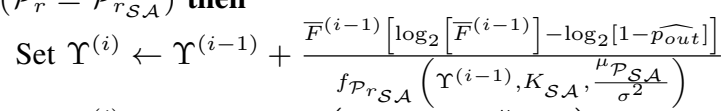

Set $\bar{F}^{(i)} \leftarrow 1-F_{\mathcal{P}_{\mathcal{S}_{\mathcal{A}}}}\left(\Upsilon^{(i)}, K_{\mathcal{S A}}, \frac{\mu_{\mathcal{P}_{\mathcal{S} \mathcal{A}}}}{\sigma^{2}}\right)$

14: $\quad$ else if $\left(\mathcal{P}_{r}=\min \left\{\mathcal{P}_{r_{\mathcal{S R}}}, \mathcal{P}_{r_{\mathcal{R} A}}+\mathcal{P}_{r_{\mathcal{S A}}}\right\}\right)$ then

$15:$

Set $\Upsilon^{(i)} \leftarrow \Upsilon^{(i-1)}+\left[\log _{2}\left[\bar{F}^{(i-1)}\right]-\log _{2}\left[1-\widehat{p_{\text {out }}}\right]\right]$

$\times \bar{F}^{(i-1)}\left[{\overline{F_{b}}}^{(i-1)} f_{\mathcal{P}_{r_{\mathcal{S} \mathcal{R}}}}\left(\Upsilon^{(i-1)}, K_{\mathcal{S R}}, \frac{\mu_{\mathcal{P}}{ }_{\mathcal{S} \mathcal{R}}}{\sigma^{2}}\right)+{\overline{F_{a}}}^{(i-1)}\right.$

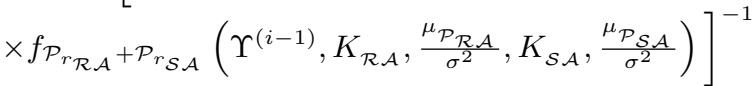

16: $\quad$ Set ${\overline{F_{a}}}^{(i)} \leftarrow 1-F_{\mathcal{P}_{r_{\mathcal{S}}}}\left(\Upsilon^{(i)}, K_{\mathcal{S R}}, \frac{\mu_{\mathcal{P} \mathcal{S} \mathcal{R}}}{\sigma^{2}}\right), \quad{\overline{F_{b}}}^{(i)} \leftarrow$

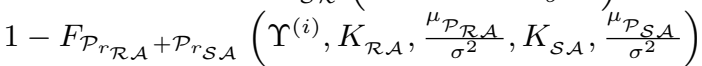

17: $\quad$ Set $\bar{F}^{(i)} \leftarrow{\overline{F_{a}}}^{(i)} \cdot{\overline{F_{b}}}^{(i)}$

18: until $\left(\left|\widehat{p_{\text {out }}}-\left(1-\bar{F}^{(i)}\right)\right| \leq \xi\right)$

merical results, we have found that on an average Algorithm 2 converges to acceptable tolerance $\xi$ in less than 20 iterations.

\section{E. Some Additional Insights on Key System Parameters}

1) Deciding $N_{s}$ Slots Dedicated for RF-ET: Since the endto-end ET efficiency is very low, we need to allocate sufficient time for ET so that both $\mathcal{S}$ and $\mathcal{R}$ have sufficient harvested energy to carry out uplink IT at a desirably rate $R_{0}$. The rate of change of $\bar{\tau}$ for NR mode with $N_{s}$ is:

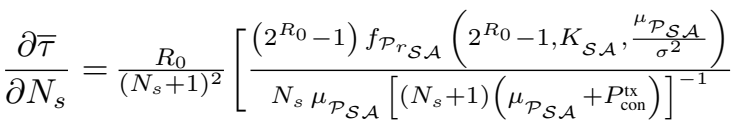

$$
\begin{aligned}
& \left.-\left(1-F_{\mathcal{P}_{r_{\mathcal{S A}}}}\left(2^{R_{0}}-1, K_{\mathcal{S A}}, \frac{\mu_{\mathcal{P}_{\mathcal{S} \mathcal{A}}}}{\sigma^{2}}\right)\right)\right] \text {. }
\end{aligned}
$$

From (33) we note that for low values of $R_{0}, \frac{\partial \bar{\tau}}{\partial N_{s}}<0$, implying that $\bar{\tau}$ in NR is a decreasing function of $N_{s}$ because for low $R_{0}$, PDF $f_{\mathcal{P}_{r_{\mathcal{S A}}}}$ is lower than $\mathrm{CCDF}\left[1-F_{\mathcal{P}_{r_{\mathcal{S A}}}}\right]$ and thus $N_{s}$ can be set as the minimum, i.e., 2 slots. However if $R_{0}$ is high for meeting the demands of high QoS applications, then $\bar{\tau}$ initially increases till $N_{s}=N_{s}^{*}$ and for $N_{s}>N_{s}^{*}$ it decreases with increased $N_{s}$. Here the optimal $N_{s}$ for NR, denoted by $N_{s}^{*}$, is obtained by solving $\frac{\partial \bar{\tau}}{\partial N_{s}}=0$. 
2) Insights on Optimal Relay Placement (ORP): Although this work focuses on solving the dilemma of $\mathcal{R}$ on whether to cooperate in downlink ER to $\mathcal{S}$ or uplink IR to $\mathcal{A}$ based on its relative placement between $\mathcal{A}$ and $\mathcal{S}$, here we give insights on ORP for different relaying modes.

For ER mode, detailed investigation on the ORP in twohop RF-ET was carried out in [5]. It was observed that ORP, always lying in the constructive interference region, depends on the end-to-end distance $d_{\mathcal{A S}}$. If $d_{\mathcal{A S}}$ is relatively low then ORP lies in the constructive interference region closer to the RF-EH device $\mathcal{S}$, whereas if $d_{\mathcal{A S}}$ is relatively high then ORP lies closer to RF source $\mathcal{A}$. We have obtained similar results as plotted in Fig. 6 and discussed in Section VII-B.

Regarding IR mode, it is difficult to obtain the closed-form results for ORP due to high composite non-linearity. However by exploiting the behavior of DF-IR protocol, we provide a suboptimal RP solution that provides tight approximation to the global-ORP. As the DF-IR performance is bottlenecked by the minimum of the SNR of $\mathcal{S}$-to- $\mathcal{R}$ link and the SNR due to MRC, we present a suboptimal RP that improves the SNR of the bottleneck link by making the two SNRs equal. This RP solution is obtained by solving $\mathbb{E}\left[\gamma_{\mathcal{S R}}\right]=\mathbb{E}\left[\gamma_{\mathcal{S A}}+\gamma_{\mathcal{R} A}\right]$. Further as $\mathbb{E}\left[\gamma_{\mathcal{S A}}\right]>0, \mathcal{R}$ is placed closer to $\mathcal{S}$ to ensure that $\mathbb{E}\left[\gamma_{\mathcal{S R}}\right]>\mathbb{E}\left[\gamma_{\mathcal{R}_{\mathcal{A}}}\right]$ The goodness of this suboptimal solution providing insights on the features of the global optimal RP solution is investigated numerically in Section VII-B.

Finally with the above discussions on ORP in ER and IR, we note that the ORP in $\mathrm{i}^{2} \mathrm{ER}$ not only lies closer to $\mathcal{S}$ to ensure efficient IR but also it should fall in the constructive interference region to ensure efficient ER. This claim is also numerically validated later in Section VII-C.

\section{NUMERICAL RESUlTS AND DISCUSSION}

We conduct numerical investigation on performance of WPCN under different relaying options: NR, ER, IR, or $\mathrm{i}^{2} \mathrm{ER}$. Unless otherwise stated, the considered system parameters are: $P_{t_{\mathcal{A}}}=30 \mathrm{dBm}, G_{\mathcal{A}}=G_{\mathcal{S}}=1 \mathrm{dBi}, G_{\mathcal{R}}=6.1 \mathrm{dBi}, \sigma^{2}=-100$ $\mathrm{dBm}, \lambda=0.328 \mathrm{~m}, y_{\mathcal{R}_{0}}=\{0.25,0.05\} \mathrm{m} \mathrm{[5]} \mathrm{for} d_{\mathcal{A S}}=\{1,2\}$ $\mathrm{m}, n=2, T=1 \mathrm{~s}, \overline{\psi^{2}}=0.175 \mathrm{rad}$ [5], $P_{\mathrm{con}}^{\mathrm{tx}}=0 \mathrm{~W}, E_{\mathrm{con}}^{\mathrm{rx}}=0.927$ $\mathrm{mJ}$ [26], $E_{\text {bit }}^{\mathrm{rx}}=93.53 \mu \mathrm{J}$ [26], $E_{i_{\mathcal{R}}}=0 \mathrm{~J}, K=10 \mathrm{~dB}$ for all the links, and tolerances as $\xi_{R_{0}}=10^{-3}, \xi=10^{-6}$.

Using (1), the piecewise linear approximation $\mathcal{P}_{h}=\mathcal{L}\left(\mathcal{P}_{r}\right)$ for $\mathcal{P}_{h}$ (in $\mathrm{mW}$ ) at the output of the commercially available Powercast P1110 RF harvester [24] can be obtained with $\mathcal{P}_{\text {th }}=\{0.282,0.501,1.0,3.548,25.119,100\} \mathrm{mW}$ as six received threshold powers dividing the harvested-received power characteristic of P1110 into $N=5$ linear pieces having slope $\mathcal{M}=\{0.857,0.786,0.485,0.733,0.465\}$ and intercept $\mathcal{C}=\{-0.223,-0.194,0.107,-0.772,5.948\} \mathrm{mW}$.

The accuracy of approximation (1) can be observed from the fact that root mean square error (RMSE) in approximating the measured results given in [5, Fig. 5(b)] is less than 0.0003 and corresponding R-square statistics value is more than 0.9997 .

\section{A. Validation of Analysis}

First, we validate the analytical expression for $E_{h_{\mathcal{S}_{\mathrm{tot}}}^{\mathrm{ER}}}^{\mathrm{ER}}$ derived using (8) and (12). Analytical results in Fig. 3 are generated

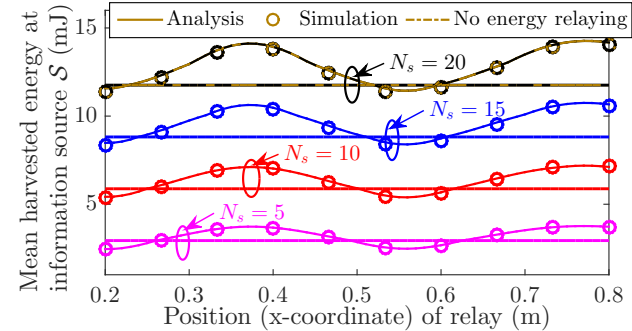

Fig. 3: Variation of $E_{h_{\mathcal{S}_{\text {tot }}}^{\mathrm{ER}}}^{\mathrm{E}}$ with relay position $\left(x_{\mathcal{R}}, 0.25 \mathrm{~m}\right)$ and $N_{s}$.

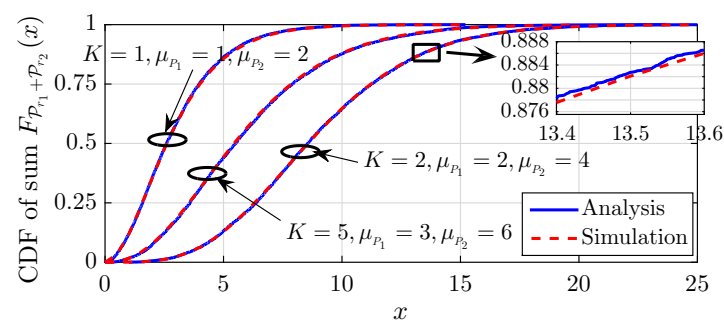

Fig. 4: Validation of expression (20) for CDF of sum of two weighted noncentral- $\chi^{2}$ random variables.

using only first 30 summands of series in (8). The simulation results on mean harvested power at $\mathcal{S}$ for varying relay position $\left(x_{\mathcal{R}}, 0.25 \mathrm{~m}\right)$ and $N_{s}$ are generated by finding mean of $10^{7}$ random realizations of harvested dc power $\mathcal{P}_{h_{\mathcal{A S}}}$ obtained by applying (1) on random received power $\mathcal{P}_{r_{\mathcal{A S}}}$ following noncentral- $\chi^{2}$ distribution. A close match between analytical and simulation results as observed in Fig. 3 validates the analysis in Section III with a RMSE of less than $10^{-4}$. From Fig. 3 it is observed that, in comparison to energy harvested $E_{h_{\mathcal{S}}}^{\mathrm{noER}}$ in no ER case, $E_{h_{\mathcal{S}_{\text {tot }}}^{\mathrm{ER}}}^{\mathrm{in}}$ in $\mathrm{ER}$ is affected by constructive and destructive interference of energy signals received from $\mathcal{A}$ and $\mathcal{R}$. However with increasing $N_{s}$, the destructive interference region decreases due to increased $E_{h_{\mathcal{R}}}$, which results in improved ER gain with higher RF-ET from $\mathcal{R}$.

Next we validate the outage analysis carried out in Section IV. We have considered only first 30 summands for each of the three series in (20) for generating analytical results depicted by solid line in Fig. 4 and different line styles in Fig. 5. We first validate expression (20) for CDF of sum of two weighted noncentral- $\chi^{2}$ random variables in Fig. 4 for different values of Rice factor $K$ and means $\mu_{\mathcal{P}_{1}}$ and $\mu_{\mathcal{P}_{2}}$. After that analytical expression (22) for $p_{\text {out }}^{\mathrm{IR}}$ is validated in Fig. 5. Monte-Carlo simulation results matching closely with

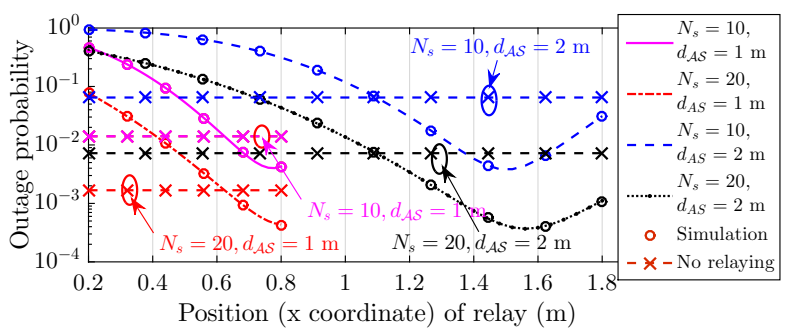

Fig. 5: Variation of $p_{\text {out }}$ in IR and NR with $x_{\mathcal{R}}, d_{\mathcal{A S}}, N_{s} . R_{0}$ is respectively 14 and $12 \mathrm{bps} / \mathrm{Hz}$ for $d_{\mathcal{A S}}$ as 1 and $2 \mathrm{~m}$. 


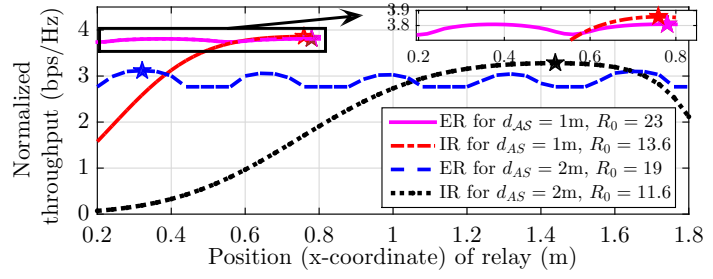

(a) Comparison for $N_{s}=5$

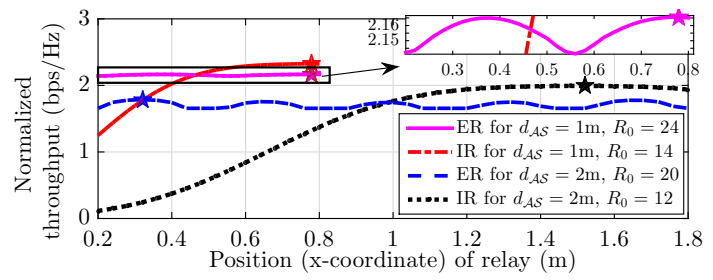

(b) Comparison for $N_{s}=10$

Fig. 6: Comparison of $\bar{\tau}$ in ER and IR modes. Stars denote ORP.

analytical results, with a RMSE of 0.0031 and 0.00034 for results plotted in Figs. 4 and 5, validate the analysis for $F_{\mathcal{P}_{r_{1}}+\mathcal{P}_{r_{2}}}$ and $p_{\text {out }}^{I R}$, respectively. Results plotted in Fig. 5 show that, IR is advantageous over NR only when $\mathcal{R}$ is closer to $\mathcal{S}$ than $\mathcal{A}$, i.e., $\frac{x_{\mathcal{R}}}{d_{\mathcal{A S}}}>\frac{1}{2}$. Minimum $p_{\text {out }}^{I R}$ is achieved when $\mathcal{R}$ is very close to $\mathcal{S}$. We also note that outage performance is improved with increasing $N_{s}$ due to increase in $P_{t_{\mathcal{R}}}$, which enhances quality of $\mathcal{R}$-to- $\mathcal{A}$ IT link.

\section{B. Energy Relaying versus Information Relaying}

We now compare the achievable normalized throughput $\bar{\tau}$ performance of ER with IR in Figs. 6(a) and 6(b) for varying $x_{\mathcal{R}}, d_{\mathcal{A S}}, R_{0}$ (in bps/Hz) with $N_{s}=5$ and $N_{s}=10$, respectively. Results plotted in Fig. 6(a) show that $\bar{\tau}$ for IR mode is more than that of ER mode if: (a) $x_{\mathcal{R}}>0.58 \mathrm{~m}$ for $d_{\mathcal{A S}}=1$ $\mathrm{m}$ and (b) $1.05<x_{\mathcal{R}}<1.6 \mathrm{~m}$ for $d_{\mathcal{A S}}=2 \mathrm{~m}$. Similarly for $N_{s}=10$ as plotted in Fig. 6(b), $\bar{\tau}$ in IR is more than that of ER $\forall x_{\mathcal{R}}>0.48 \mathrm{~m}$ if $d_{\mathcal{A S}}=1 \mathrm{~m}$ and $\forall x_{\mathcal{R}}>0.99 \mathrm{~m}$ if $d_{\mathcal{A S}}=2$ $\mathrm{m}$. This shows that ER is better than IR if $\mathcal{R}$ is positioned closer to $\mathcal{A}$, and vice-versa. Further, the $O R P$ for $I R$ is very close to information source $\mathcal{S}$. Whereas ORP in ER, which is affected by continuous constructive-destructive interference cycles, is very close to $\mathcal{S}$ for $d_{\mathcal{A S}}=1 \mathrm{~m}$, and it is very close to $\mathcal{A}$ for $d_{\mathcal{A S}}=2 \mathrm{~m}$. From Fig. 6(a) we also notice that when $\mathcal{R}$ is placed very close to $\mathcal{S}$, it may lead to weakening of $\mathcal{R}$-to- $\mathcal{A}$ link, and hence violating the upper bound $d_{\mathcal{R} \mathcal{A}}^{\max }$ on $d_{\mathcal{R A} A}$. This leads to the degraded IR performance in comparison to ER (or NR) as mentioned in Lemma 3 and Corollary 1 . For the four considered combinations of $\left(N_{s}, d_{\mathcal{A S}}\right)=$ $\{(5,1 \mathrm{~m}),(5,2 \mathrm{~m}),(10,1 \mathrm{~m}),(10,2 \mathrm{~m})\}$, the numerically found global ORP solutions for IR as plotted in Fig. 6 are $x_{\mathcal{R}}^{*}=$ $\{0.76,1.44,0.78,1.52\} \mathrm{m}$ with respective optimal throughput being $\bar{\tau}^{*}=\{3.8587,3.2803,2.3240,1.9923\}$ bps/Hz. The corresponding suboptimal RP solutions $\widehat{x_{\mathcal{R}}^{*}}$ and their respective throughput $\widehat{\bar{\tau}^{*}}$ are given by $\widehat{x_{\mathcal{R}}^{*}}=\{0.75,1.43,0.76,1.47\} \mathrm{m}$ and $\widehat{\bar{\tau}^{*}}=\{3.8586,3.2802,2.3239,1.9921\} \mathrm{bps} / \mathrm{Hz}$. Thus, the difference in throughput performance of suboptimal RP for IR mode is less than $0.0071 \%$.

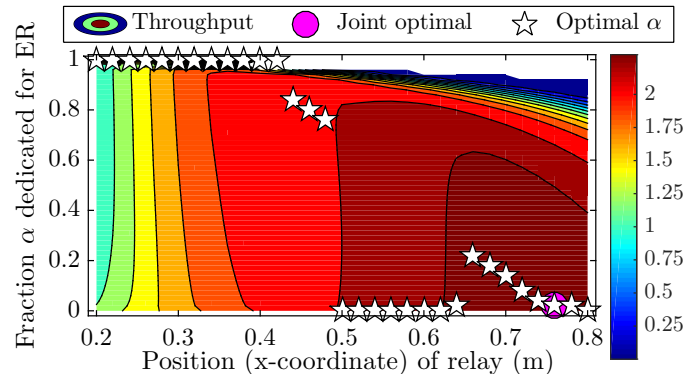

Fig. 7: Variation of $\bar{\tau}$ with $\alpha, x_{\mathcal{R}}$ for $N_{s}=10, d_{\mathcal{A S}}=1 \mathrm{~m}$. Joint optimal along with optimal $\alpha$ for each $x_{\mathcal{R}}$ also plotted.

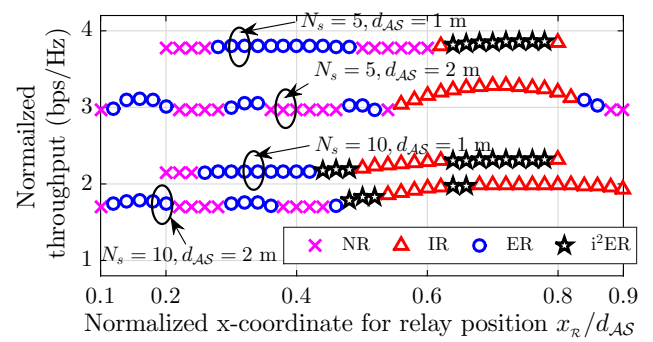

Fig. 8: Variation of $\bar{\tau}$ and optimal relaying mode with $x_{\mathcal{R}}, N_{s}, d_{\mathcal{A S}}$ for optimized $\left(\alpha^{*}, \beta^{*}\right)$ and fixed $R_{0}$.

\section{Efficient Utilization of Harvested Energy at $\mathcal{R}$}

In Fig. 7, we plot the variation of $\bar{\tau}$ with relay position $\left(x_{\mathcal{R}}, y_{\mathcal{R}_{0}}=0.25 \mathrm{~m}\right)$ and $\alpha$ for $R_{0}=14 \mathrm{bps} / \mathrm{Hz}$. Results show that for lower $x_{\mathcal{R}}, \alpha=1$ is optimal which implies that $E R$ is a better mode for low $x_{\mathcal{R}}$. As $x_{\mathcal{R}}$ increases and goes beyond $0.42 \mathrm{~m}$, optimal $\alpha$ reduces and is equal to zero (IR) for destructive interference cycle and $\alpha \neq 0$ (i $\left.{ }^{2} \mathrm{ER}\right)$ for constructive cycle (cf. zoomed plot in Fig. 6(b)). Fig. 7 shows that joint optimal solution that maximizes $\bar{\tau}$ in $i^{2} E R$ is $\left(\alpha^{*}=0.02, x_{\mathcal{R}}^{*}=0.76 m\right)$. This implies that, if relay position is controllable then $\mathcal{R}$ should be placed close to $\mathcal{S}$ with higher share of harvested energy at $\mathcal{R}$ allocated for IR. Also, the difference in throughput performance of the suboptimal RP is $0.756 \mathrm{~m}$, which is less than $0.0034 \%$.

In Fig. 8, we plot the optimal relaying mode along with achievable $\bar{\tau}$ for varying $\frac{x_{\mathcal{R}}}{d_{\mathcal{A S}}}$. Considering four combinations of $\left(N_{s}, d_{\mathcal{A S}}\right)=\{(5,1 \mathrm{~m}),(5,2 \mathrm{~m}),(10,1 \mathrm{~m}),(10,2 \mathrm{~m})\}, R_{0}=$ $\{23,19,24,20\} \mathrm{bps} / \mathrm{Hz}$ for NR and ER, and $R_{0}=\{13.6,11.6$, $14,12\} \mathrm{bps} / \mathrm{Hz}$ for IR and $\mathrm{i}^{2} \mathrm{ER}$. These values are based on maximum $R_{0}$ achievable in each mode such that resulting $p_{\text {out }} \leq 10^{-2}$. When $\mathcal{R}$ is close to $\mathcal{A}$, i.e., $\frac{x_{\mathcal{R}}}{d_{\mathcal{A S}}}<0.44$, there are only two optimal modes possible: ER for constructive interference regions and $N R$ for destructive interference regions. In contrast, as shown in Fig. 8, IR or $i^{2} E R$ is selected as optimal mode when $\mathcal{R}$ is close to center or $\mathcal{S}$. When $N_{s}$ is sufficiently high and $\mathcal{R}$ is placed close to $\mathcal{S}$,WPCN can benefit from both $I R$ and ER ( $i^{2} E R$ mode is optimal) in constructive regions. IR is optimal when $\mathcal{R}$ is close to $\mathcal{S}$ and $d_{\mathcal{A S}}$ is large which leads to need for alternate IR link due to weakening of $\mathcal{S}$-to- $\mathcal{A}$ link.

For the relay positions in Fig. 8 where NR was selected (as denoted by " $\times$ " mark), we next investigate the effect of energy accumulation at $\mathcal{R}$ due to the unused harvested 
TABLE II: Investigting the effect of energy accumulation at $\mathcal{R}$ during the NR modes plotted in Fig. 8.

\begin{tabular}{|c|c|c|c|c|c|c|c|c|c|c|c|c|c|c|c|}
\hline \multicolumn{4}{|c|}{ I: $N_{s}=5, d_{\mathcal{A S}}=1 \mathrm{~m}$} & \multicolumn{4}{|c|}{ II: $N_{s}=10, d_{A S}=1 \mathrm{~m}$} & \multicolumn{4}{|c|}{ III: $N_{s}=5, d_{\mathcal{A S}}=2 \mathrm{~m}$} & \multicolumn{4}{|c|}{ IV: $N_{s}=10, d_{\mathcal{A S}}=2 \mathrm{~m}$} \\
\hline S. No. & $x_{\mathcal{R}}(\mathbf{m})$ & Mode & Blocks & S. No. & $x_{\mathcal{R}}(\mathbf{m})$ & Mode & Blocks & S. No. & $x_{\mathcal{R}}(\mathbf{m})$ & Mode & Blocks & S. No. & $x_{\mathcal{R}}(\mathbf{m})$ & Mode & Blocks \\
\hline 1 & 0.20 & ER & 5 & 1 & 0.20 & ER & 3 & 5 & 0.52 & ER & 4 & 1 & 0.20 & ER & 2 \\
\hline 2 & 0.22 & ER & 5 & 2 & 0.22 & ER & 3 & 6 & 0.56 & ER & 7 & 2 & 0.44 & ER & 3 \\
\hline 3 & 0.24 & ER & 3 & 3 & 0.24 & ER & 2 & 7 & 0.76 & ER & 20 & 3 & 0.48 & ER & 6 \\
\hline 4 & 0.26 & ER & 2 & & & & & 8 & 0.80 & ER & 22 & 4 & 0.52 & ER & 2 \\
\hline 5 & 0.50 & ER & 2 & & $N_{s}=5$ & $A S=$ & & 9 & 0.84 & ER & 9 & 5 & 0.56 & ER & 2 \\
\hline 6 & 0.52 & ER & 4 & S. No. & $x_{\mathcal{R}}(\mathbf{m})$ & Mode & Blocks & 10 & 0.88 & ER & 2 & 6 & 0.76 & ER & 4 \\
\hline 7 & 0.54 & ER & 6 & 1 & 0.20 & ER & 4 & 11 & 0.92 & ER & 2 & 7 & 0.8 & ER & 10 \\
\hline 8 & 0.56 & ER & 7 & 2 & 0.44 & ER & 5 & 12 & 1.08 & ER & 6 & 8 & 0.84 & ER & 11 \\
\hline 9 & 0.58 & ER & 6 & 3 & 0.48 & ER & 12 & 13 & 1.76 & IR & 2 & 9 & 0.88 & ER & 5 \\
\hline 10 & 0.60 & ER & 4 & 4 & 0.52 & ER & 11 & 14 & 1.80 & IR & 2 & & & & \\
\hline
\end{tabular}
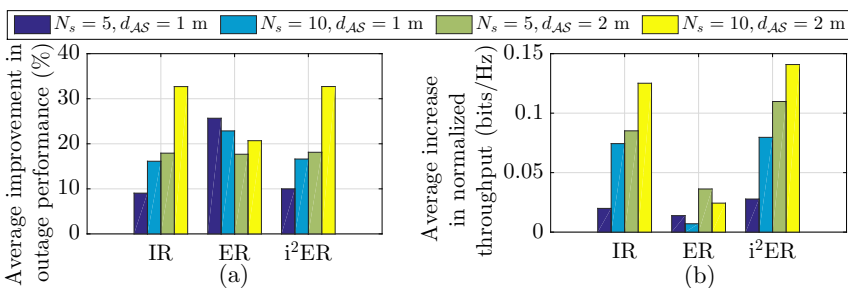

Fig. 9: Enhancement in $p_{\text {out }}$ and $\bar{\tau}$ for IR, ER, and $i^{2}$ ER over NR.

energy $E_{i_{\mathcal{R}}}$ that is available at the beginning of future transmission block(s) of these NR cases. The corresponding results showing the number of NR transmission blocks after which a relay position becomes suitable for relaying (ER/IR) due to accumulated energy are tabulated in Table II. The optimal relaying mode that is eventually selected for utilizing all the accumulated energy over previous NR blocks is also listed.

The results in Table II show that mostly ER mode is selected when the accumulated energy is sufficient such that the feasibility conditions for ER mode (as discussed in Section V-A) are met, implying that ER mode is better than NR. IR mode is selected only in the two cases where the x-coordinate $x_{\mathcal{R}}$ of relay placement was such that $\mathcal{R}$ was placed very close to $\mathcal{S}$ and the feasibility conditions for IR mode (as discussed in Section V-B) are met. Also, the number of consecutive transmission blocks where NR was selected before the unused energy harvested over these blocks became sufficient for making use of the RF-EH relay $\mathcal{R}$ in terms of ER, IR, or $\mathrm{i}^{2} \mathrm{ER}$ vary from 2 (minimum) to as high as 22 blocks. Although there is no general trend, it is noted that lower end-to-end distance $d_{\mathcal{A S}}$ cases require lesser NR transmission blocks for making use of unused harvested energy at $\mathcal{R}$ for relaying. On the other hand, the larger number of blocks are required to have sufficient amount of energy at $\mathcal{R}$ which ensures that $\alpha>4 \alpha_{g_{\mathrm{ER}}}$ (cf. Remark 1), or in other words for ER with $\alpha=\beta=1$, to ensure that $\mu_{\mathcal{P}_{\mathcal{R} S}}=\left(E_{h_{\mathcal{R}}}+E_{i_{\mathcal{R}}}\right) \frac{G_{t_{\mathcal{R}}} G_{\mathcal{S}}}{\left(d_{\mathcal{R S}}\right)^{n}}\left(\frac{\lambda}{4 \pi}\right)^{2}>\mu_{0}^{2} \mu_{\mathcal{P}_{\mathcal{A S}}}$.

Fig. 9 plots average improvement $\left(\frac{\max \left\{p_{\text {out }}^{\text {nolR }}-p_{\text {out }}^{\text {mode }}, 0\right\} \times 100 \%}{p_{\text {out }}^{\text {nol }}}\right)$ in $p_{\text {out }}$ and average increase $\left(\max \left\{\bar{\tau}^{\text {mode }}-\bar{\tau}^{\mathrm{NR}}, 0\right\}\right)$ in $\bar{\tau} \forall$ mode $=\left\{E R, I R, i^{2} E R\right\}$. Results show that $i^{2} E R$ offers the maximum improvement in $p_{\text {out }}$ and $\bar{\tau}$ over NR, and is closely followed by IR. It is noted that, due to cooperative diversity maximum achievable $\bar{\tau}, \frac{2 R_{0}}{N_{s}+2}=\{3.886,3.314,2.333,2\}$ in IR and $\mathrm{i}^{2} \mathrm{ER}$, is slightly more than the achievable $\bar{\tau}$, $\frac{R_{0}}{N_{s}+1}=\{3.833,3.167,2.182,1.818\}$ in ER and NR modes at four combinations of $\left(N_{s}, d_{\mathcal{A S}}\right)$. Due to this, IR offers a higher

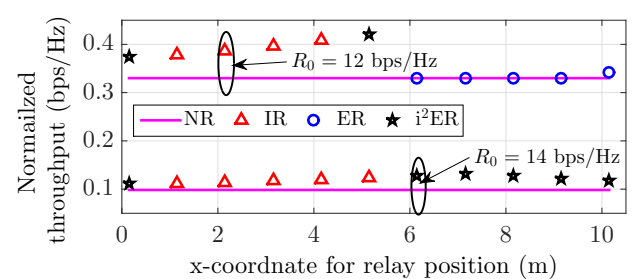

Fig. 10: Variation of $\bar{\tau}^{*}$ for advanced RF-EH circuits.

throughput gain than ER (Fig 9(b)) and in the process lower outage improvement (Fig 9(a)) for $d_{\mathcal{A S}}=1 \mathrm{~m}$. Although ER provides lesser increase in $\bar{\tau}$ than IR, ER offers more stable performance (cf. Figs. 6(a), 6(b)). IR is advantageous when $\mathcal{R}$ is close to $\mathcal{S}$, specifically when $\left(d_{\mathcal{S R}}, d_{\mathcal{R A}}\right)$ lies in the set $\mathbb{S}_{\mathrm{IR}}$ defined in Corollary 1. The importance of $I R, E R$, and $i^{2} E R$ increases with increasing WPCN range $d_{\mathcal{A S}}$ because direct link gets weakened and the role of additional relay link becomes more prominent. From Fig. 9(b) we also observe that, though increasing $N_{s}$ improves IR and $i^{2} E R$ gains, it actually degrades ER performance because this increase in energy is not sufficient to provide high rate over increased $N_{s}$ slots.

\section{Investigating the Impact of Advanced RF-EH Circuits}

Now we investigate performance of the proposed protocol and optimized solutions for larger end-to-end RF-ET range $d_{\mathcal{A S}}$ achieved with the help of advanced RF-EH circuits which are currently under research [22], [23] and will be available commercially in future. With these advancements in RF-EH technology, we can efficiently harvest input RF powers as low as $-20 \mathrm{dBm}$ at a rectification efficiency of 0.5 (or $50 \%$ ). Also this RF-to-dc rectification efficiency does not degrade and can be maintained constant at 0.5 for all RF input powers $\geq-20$ $\mathrm{dBm}$. With this setting, RF-ET range $d_{\mathcal{A S}}$ can be improved from $2 \mathrm{~m}$ to $10.3 \mathrm{~m}$. For this improved RF-ET range we have plotted the optimal relaying mode along with maximum normalized throughput $\bar{\tau}^{*}$ for varying relay positions and $R_{0}$ in Fig. 10. Here we have also considered setting $N_{s}$ as $\widehat{N_{s}^{*}}$ to meet a rate requirement of at least $R_{0}$ with high probability greater than 0.99 even for NR mode. Following the discussion in Section VI-E1, $\widehat{N_{s}^{*}}$ is respectively obtained as 35 slots and 140 slots for $R_{0}=12 \mathrm{bps} / \mathrm{Hz}$ and $R_{0}=14 \mathrm{bps} / \mathrm{Hz}$.

Results plotted in Fig. 10 show that, for larger $d_{\mathcal{A S}}=10.3$ $\mathrm{m}$ the performance of both ER and IR gets affected. On one hand, the gap between ER and NR is reduced due to decrease in ER efficiency because of low energy harvested at $\mathcal{R}$ over 


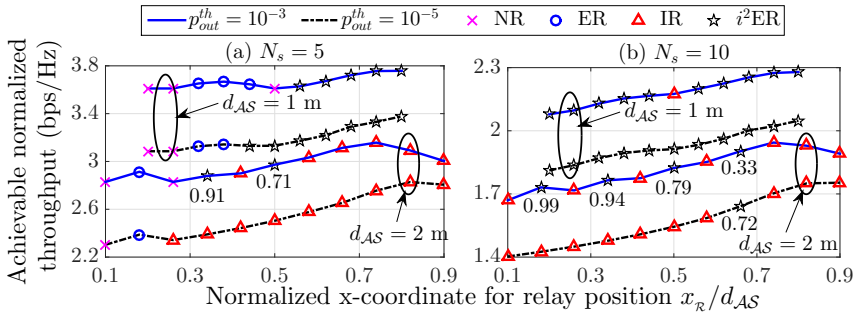

Fig. 11: Variation of maximized $\bar{\tau}^{*}$ and optimal relaying mode with $x_{\mathcal{R}}, N_{s}, d_{\mathcal{A S}}$ for jointly-optimized $\left(R_{0}^{*}, \alpha^{*}, \beta^{*}\right)$.
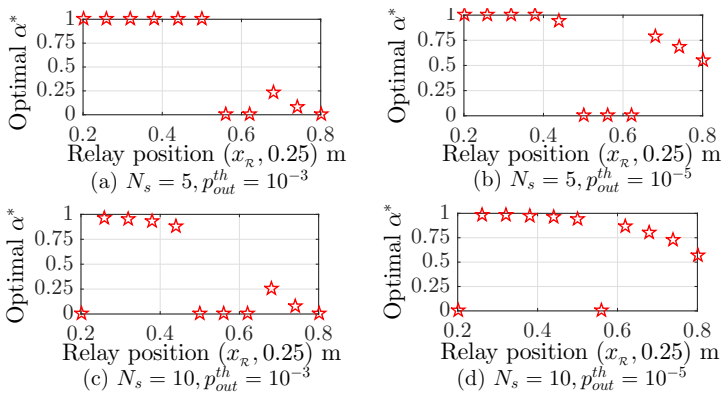

Fig. 12: Investigating $\alpha^{*}$ in jointly-optimized solution of (P1).

larger $\mathcal{A}$-to- $\mathcal{R}$ distance and low energy delivered to $\mathcal{S}$ over larger $\mathcal{R}$-to- $\mathcal{S}$ distance. Whereas, the performance of IR and $\mathrm{i}^{2} \mathrm{ER}$ with low $\widehat{N_{s}^{*}}=35$ slots is poorer than ER for $R_{0}=$ $12 \mathrm{bps} / \mathrm{Hz}$ because energy harvested at $\mathcal{R}$ over $\widehat{N_{s}^{*}}$ slots for larger $\mathcal{A}$-to- $\mathcal{R}$ distances, denoted by larger $x_{\mathcal{R}}$, is not sufficient to meet the decoding costs. However when $\widehat{N}_{s}^{*}=140$ slots for meeting $R_{0}=140 \mathrm{bps} / \mathrm{Hz}$, IR and $\mathrm{i}^{2} \mathrm{ER}$ perform better than ER even for larger $\mathcal{A}$-to- $\mathcal{R}$ distances because now the energy harvested over such large number of slots is sufficient for meeting the decoding costs.

\section{E. Performance for Jointly-Optimized $\left(R_{0}^{*}, \alpha^{*}, \beta^{*}\right)$ in (P1)}

Now we investigate the maximum normalized throughput $\bar{\tau}^{*}$ performance achieved by jointly optimizing $\left(R_{0}^{*}, \alpha^{*}, \beta^{*}\right)$ in (P1) for different QoS requirements represented in terms of varying outage probability threshold $p_{\text {out }}^{\text {th }}=\left\{10^{-2}, 10^{-3}, 10^{-4}\right.$, $\left.10^{-5}, 10^{-6}\right\}$. Variation of optimized $\bar{\tau}^{*}$ along with optimal mode with $p_{\text {out }}^{\text {th }}, x_{\mathcal{R}}, N_{s}$, and $d_{\mathcal{A S}}$ is potted in Fig. 11. It is observed that $\bar{\tau}^{*}$ for $p_{\text {out }}^{\text {th }}=10^{-5}$ is $13.29 \%$ lower than for $p_{\text {out }}^{\text {th }}=10^{-3}$ due to reduced $R_{0}^{*}$. Comparing with results in Fig. 8 having fixed $R_{0}$, results in Fig. 11 with optimized $R_{0}^{*}$ show that with decreasing $p_{\text {out }}^{\text {th }}$ (stricter QoS requirement), $I R$ and $i^{2} E R$ modes become more useful for meeting high $R_{0}$ requirements. The corresponding optimal $\alpha^{*}$ is plotted in Fig. 12 for $d_{\mathcal{A S}}=1 \mathrm{~m}$. Optimal $\alpha^{*}$ for $d_{\mathcal{A S}}=2 \mathrm{~m}$ is noted in Fig. 11 itself with NR, ER, and IR having optimal $\left(\alpha^{*}, \beta^{*}\right)$ as $(-, 0),(1,1),(0,1)$, respectively. From Figs. 11 and 12 , it is observed that in general optimal $\alpha^{*}$ decreases with increased distance $d_{\mathcal{A R}}=\sqrt{x_{\mathcal{R}}^{2}+y_{\mathcal{R}_{0}}^{2}}$ between $\mathcal{A}$ and $\mathcal{R}$, except during destructive interference cycle (cf. Figs. 6(a) and 6(b)) where $\alpha^{*} \approx 0$. From Fig. 12 it also is noted that optimal $\alpha^{*}$ in $\mathrm{i}^{2} \mathrm{ER}$ for $d_{\mathcal{A S}}=1 \mathrm{~m}$ increases with decreased $p_{\text {out }}^{\text {th }}$.

Next, we investigate the tradeoff between the optimized $\bar{\tau}^{*}$ and acceptable outage probability (QoS) requirement $p_{\text {out }}^{\text {th }}$.

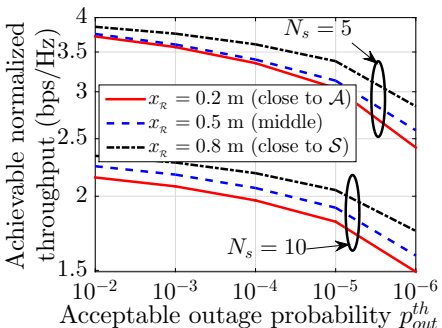

Fig. 13: Tradeoff between optimized $\bar{\tau}$ and outage requirement $p_{\text {out }}^{\text {th }}$ in (P1) for varying $x_{\mathcal{R}}, N_{s}$.

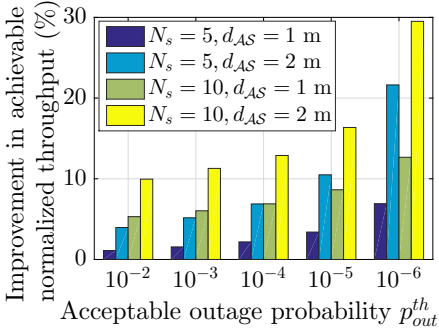

Fig. 14: Improvement provided by optimized $\mathrm{i}^{2} \mathrm{ER}$ model over benchmark WPCN model [21].
Results plotted in Fig. 13 for $d_{\mathcal{A S}}=1 \mathrm{~m}$ show that achievable normalized throughput $\bar{\tau}^{*}$ respectively decreases by $30.9 \%$ and $28.3 \%$ for $N_{s}=5$ and $N_{s}=10$ slots when $p_{\text {out }}^{\text {th }}$ is increased from $10^{-2}$ to $10^{-6}$. Further it is also noted that $\mathcal{R}$ placed closer to $\mathcal{S}$ (i.e., higher $x_{\mathcal{R}}$ ) helps in achieving higher $\bar{\tau}^{*}$, with $N_{s}=5$ providing higher $\bar{\tau}^{*}$ than $N_{s}=10$ slots.

Finally, we present the throughput performance enhancement results achieved with the help of optimized $i^{2}$ ER model over the benchmark WPCN model with NR. Results plotted in Fig. 14 show that improvement in optimal $\bar{\tau}^{*}$ increases monotonically with increased $Q o S$ requirement (i.e., decreased $\left.p_{\text {out }}^{\text {th }}\right)$ for all four combinations of $\left(N_{s}, d_{\mathcal{A S}}\right)$. In fact as $p_{\text {out }}^{\text {th }}$ decreases from $10^{-2}$ to $10^{-6}$, optimized $i^{2} E R$ improves the throughput performance of NR from $10 \%$ to up to $30 \%$.

\section{CONCLUding Remarks}

This paper has investigated efficient utilization of harvested energy at RF harvesting relay $\mathcal{R}$ for either downlink ER, or uplink IR, or $\mathrm{i}^{2} \mathrm{ER}$, to maximize the normalized throughput $\bar{\tau}$ for information transfer from an energy-constrained source $\mathcal{S}$ to an energy-surplus HAP $\mathcal{A}$. While considering $\mathrm{i}^{2} \mathrm{ER}$, closedform expressions for mean harvested energy at $\mathcal{S}$ and outage probability at HAP $\mathcal{A}$ with MRC over Rician channels have been derived. Using these expressions, analytical insights on optimal relaying mode have been provided along with globaloptimal sharing of harvested energy at $\mathcal{R}$ for maximizing $\bar{\tau}$ while ensuring very low outage probability $p_{\text {out }}^{\text {th }}$ in achieving rate $R_{0}$. The analysis has been validated by simulation results. Via numerical investigation it has been observed that, when $\mathcal{R}$ is close to $\mathcal{A}$, ER and NR are the optimal modes. On other hand, when $\mathcal{R}$ is close to $\mathcal{S}$, IR and $\mathrm{i}^{2} \mathrm{ER}$ are more beneficial. In general, $\mathcal{R}$ positioned closer to $\mathcal{S}$, with higher share of harvested energy allocated for IR, provides higher $\bar{\tau}$. Overall in comparison to benchmark NR mode, $\mathrm{i}^{2} \mathrm{ER}$ having advantages of both IR and ER offers an average outage improvement of $22 \%$ for fixed $R_{0}$ and up to $30 \%$ improvement in $\bar{\tau}$ by jointlyoptimizing $\left(R_{0}^{*}, \alpha^{*}, \beta^{*}\right)$ for $p_{\text {out }}^{\text {th }}=10^{-6}$. Thus, this paper provides a benchmark for further investigation on optimized $\mathrm{i}^{2} \mathrm{ER}$ aspects for improving the performance of WPCNs.

\section{APPENDiX A}

PROOF OF LEMMA 3

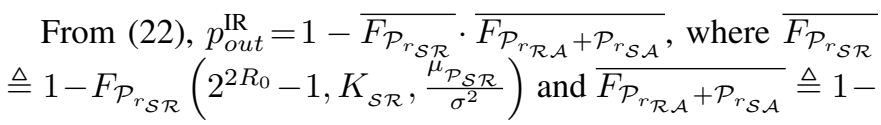




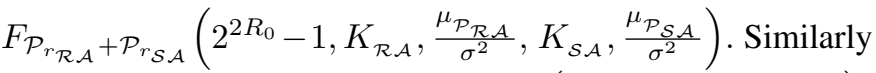
from (24), $p_{\text {out }}^{\text {nolR }}=1-\overline{F_{\mathcal{P}_{r_{\mathcal{A}}}}}=F_{\mathcal{P}_{r_{\mathcal{S A}}}}\left(2^{R_{0}}-1, K_{\mathcal{S A}}, \frac{\mu_{\mathcal{P}_{\mathcal{S A}}}}{\sigma^{2}}\right)$. As $0 \leq \overline{F_{\mathcal{P}_{r_{\mathcal{A}}}+\mathcal{P}_{r_{\mathcal{S A}}}}}, \overline{F_{\mathcal{P}_{r_{\mathcal{S}}}}} \leq 1, p_{\text {out }}^{\mathrm{IR}}<p_{\text {out }}^{\text {nolR }}$ can be ensured by showing that $\overline{F_{\mathcal{P}_{r_{\mathcal{A}}}}} \leq \min \left\{\overline{F_{\mathcal{P}_{r_{\mathcal{R}}}}}, \overline{F_{\mathcal{P}_{r_{\mathcal{R A}}}+\mathcal{P}_{r_{\mathcal{S A}}}}}\right\}$. Considering same Rice factor for all links, i.e., $K_{\mathcal{S A}}=$ $K_{\mathcal{S R}}=K_{\mathcal{R A}}=K$, first we derive the condition for

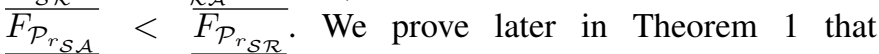
$\overline{F_{\mathcal{P}_{r_{\mathcal{A}}}}}$ and $\overline{F_{\mathcal{P}_{\boldsymbol{S}_{\mathcal{R}}}}}$, both following same distribution, are increasing functions of their respective means $\mathbb{E}\left[\gamma_{\mathcal{S}_{\mathcal{A}}}\right]$ and $\mathbb{E}\left[\gamma_{\mathcal{S R}}\right]$. As $\overline{F_{\mathcal{P}_{r_{\mathcal{A}}}}}=\operatorname{Pr}\left(\log _{2}\left(1+\gamma_{\mathcal{S A}}\right)>R_{0}\right)$ and $\overline{F_{\mathcal{P}_{r_{\mathcal{R}}}}}$ $=\operatorname{Pr}\left(\frac{1}{2} \log _{2}\left(1+\gamma_{\mathcal{S R}}\right)>R_{0}\right), \overline{F_{\mathcal{P}_{r_{\mathcal{S A}}}}}<\overline{F_{\mathcal{P}_{r_{\mathcal{S R}}}}}$ is equivalent to showing $\mathbb{E}\left[\log _{2}\left(1+\gamma_{\mathcal{S A}}\right)\right] \stackrel{<\mathcal{E}}{<}\left[\frac{1}{2} \log _{2}\left(1+\gamma_{\mathcal{S R}}\right)\right]$. Since it is difficult to obtain closed-form expression for $\mathbb{E}\left[\log _{2}\left(1+\gamma_{\mathcal{S A}}\right)\right]$, we consider an alternative. From Jensen's inequality [35] for expectation of concave transformation $g(\cdot)$ of random variable $X$, we note that $\mathbb{E}[g(X)] \leq g(\mathbb{E}[X])$. As $\log _{2}(\cdot)$ is an increasing concave function, $\overline{F_{\mathcal{P}_{\mathcal{S}_{\mathcal{A}}}}}<\overline{F_{\mathcal{P}_{r_{\mathcal{S R}}}}}$ requires that $\log _{2}\left(1+\mathbb{E}\left[\gamma_{\mathcal{S A}}\right]\right)<\frac{1}{2} \log _{2}\left(1+\mathbb{E}\left[\gamma_{\mathcal{S R}}\right]\right)$, which on simplification results in $\mathbb{E}\left[\gamma_{\mathcal{S R}}\right]>\mathbb{E}\left[\gamma_{\mathcal{S A}}\right]\left(\mathbb{E}\left[\gamma_{\mathcal{S A}}\right]+2\right)$.

Now we prove part (ii) of the lemma. As $\overline{F_{\mathcal{P}_{r_{\mathcal{A}}}+\mathcal{P}_{r_{\mathcal{S}}}}}=\operatorname{Pr}\left(\frac{1}{2} \log _{2}\left(1+\gamma_{\mathcal{S}_{\mathcal{A}}}+\gamma_{\mathcal{R}_{\mathcal{A}}}\right)>R_{0}\right), \overline{F_{\mathcal{P}_{\mathcal{S}_{\mathcal{A}}}}}$ $<\frac{\overline{\mathcal{R}_{\mathcal{A}}}+\mathcal{P}_{\boldsymbol{P}_{\mathcal{R} \mathcal{A}}}+\mathcal{P}_{r_{\mathcal{S A}}}}{\text { is }}$ is equivalent to $\operatorname{Pr}\left(\log _{2}\left(1+\gamma_{\mathcal{S A}}\right)>R_{0}\right)<$ $\operatorname{Pr}\left(\log _{2}\left(\sqrt{1+\gamma_{\mathcal{S A}}+\gamma_{\mathcal{R A}}}\right)>R_{0}\right)$. Due to monotonically increasing nature of $\log (\cdot)$ function, this condition implies that we need to show that the random variable $1+\gamma_{\mathcal{S A}}$ is lower than random variable $\sqrt{1+\gamma_{\mathcal{S A}}+\gamma_{\mathcal{R A}}}$, where $\gamma_{\mathcal{S A}}, \gamma_{\mathcal{R A}} \geq 0$. Therefore, for a given $\gamma_{\mathcal{S A}}$ this condition reduces to $\gamma_{\mathcal{R A}}>\gamma_{\mathcal{S A}}\left(1+\gamma_{\mathcal{S A}}\right)$. To gain further insight, we consider the expected approximation of this relationship given by: $\mathbb{E}\left[\gamma_{\mathcal{R}_{\mathcal{A}}}\right]>\mathbb{E}\left[\gamma_{\mathcal{S} \mathcal{A}}\right]\left(1+\mathbb{E}\left[\gamma_{\mathcal{S} \mathcal{A}}\right]\right)$. This condition poses an upper bound on $\alpha$ given by:

$$
\begin{aligned}
\alpha< & \frac{\overline{\mathcal{P}_{h_{\mathcal{A}}}} N_{s}+E_{i_{\mathcal{R}}}-P_{\mathrm{con}}^{\mathrm{tx}}-E_{\mathrm{con}}^{\mathrm{rx}}-R_{0} E_{\mathrm{bit}}^{\mathrm{rx}}}{\overline{\mathcal{P}_{h_{\mathcal{A R}}}} N_{s}} \\
& -\frac{\left(N_{s} \overline{\mathcal{P}_{h_{\mathcal{A}}}}-P_{\mathrm{con}}^{\mathrm{tx}}\right) G_{\mathcal{A}}\left(1+\mathbb{E}\left[\gamma_{\mathcal{S}}\right]\right)\left(d_{\mathcal{S R}}\right)^{n}}{\overline{\mathcal{P}_{h_{\mathcal{A}}}} N_{s} G_{\mathcal{R}}\left(d_{\mathcal{S} \mathcal{A}}\right)^{n}} .
\end{aligned}
$$

As minimum $\alpha=0$ can be achieved in IR mode, it gives the maximum value of $\mathbb{E}\left[\gamma_{\mathcal{R A}}\right]$.

\section{APPENDIX B \\ PROOF OF THEOREM 1}

1) Log-concavity of $\overline{F_{\mathcal{P}_{r_{\mathcal{R}}}}}$ in $\mathbb{E}\left[\gamma_{\mathcal{S R}}\right]$ : Using composite definition for CCDF $\overline{F_{\mathcal{P}_{r_{\mathcal{R}}}}}=\left(\mathcal{F}_{1} \circ \mathcal{F}_{2}\right)\left(\mu_{\mathcal{P}_{\mathcal{S R}}}\right)$, where $\mathcal{F}_{1}($ $\left.\mathcal{F}_{2}\left(\mu_{\mathcal{P}_{\mathcal{S R}}}\right)\right)=Q_{1}\left(\sqrt{2 K_{\mathcal{S R}}}, \sqrt{\mathcal{F}_{2}\left(\mu_{\mathcal{P}_{\mathcal{S R}}}\right)}\right)$ and $\mathcal{F}_{2}\left(\mu_{\mathcal{P}_{\mathcal{S R}}}\right) \triangleq$ $\frac{2\left(K_{\mathcal{S R}}+1\right) \sigma^{2}}{\left(2^{2 R_{0}}-1\right)^{-1} \mu_{\mathcal{P}_{\mathcal{S R}}}}$, log-concavity of $\overline{F_{\mathcal{P}_{r_{\mathcal{S R}}}}}$ in $\mu_{\mathcal{P}_{\mathcal{S R}}}$ requires:

$$
\begin{aligned}
\frac{\partial^{2} \log \left(\mathcal{F}_{1}\left(\mathcal{F}_{2}\right)\right)}{\partial \mu_{\mathcal{P}_{\mathcal{S R}}}{ }^{2}}= & \left(\frac{\partial \mathcal{F}_{2}}{\partial \mu_{\mathcal{P}_{\mathcal{S R}}}}\right)^{2}\left[\frac{1}{\mathcal{F}_{1}} \frac{\partial^{2} \mathcal{F}_{1}}{\partial \mathcal{F}_{2}^{2}}-\left(\frac{1}{\mathcal{F}_{1}} \frac{\partial \mathcal{F}_{1}}{\partial \mathcal{F}_{2}}\right)^{2}\right] \\
& +\frac{1}{\mathcal{F}_{1}} \frac{\partial^{2} \mathcal{F}_{2}}{\partial \mu_{\mathcal{P}_{\mathcal{S R}}}{ }^{2}} \frac{\partial \mathcal{F}_{1}}{\partial \mathcal{F}_{2}} \leq 0 .
\end{aligned}
$$

As generalized Marcum Q-function $Q_{\nu}(\sqrt{a}, \sqrt{b})$ is $\log$ concave in $b \in[0,1) \forall \nu \geq 1, a \geq 0$ [36], [37], nonnegativity of $K_{\mathcal{S R}}, R_{0}$, and $\frac{\sigma^{2}}{\mu_{\mathcal{S} \mathcal{R}}}$ implies log-concavity of $\mathcal{F}_{1}\left(\mathcal{F}_{2}\right)=Q_{1}\left(\sqrt{2 K_{\mathcal{S R}}}, \sqrt{\mathcal{F}_{2}}\right)$ in $\mathcal{F}_{2}$. This shows that first summation term in (B.1) is non-positive because $\frac{\partial^{2} \log \left(\mathcal{F}_{1}\right)}{\partial \mathcal{F}_{2}^{2}}=$ $\frac{1}{\mathcal{F}_{1}} \frac{\partial^{2} \mathcal{F}_{1}}{\partial \mathcal{F}_{2}^{2}}-\left(\frac{1}{\mathcal{F}_{1}} \frac{\partial \mathcal{F}_{1}}{\partial \mathcal{F}_{2}}\right)^{2} \leq 0$. Also from [37], $\overline{F_{\mathcal{P}_{\boldsymbol{S}_{\mathcal{R}}}}}=$ $\mathcal{F}_{1}\left(\mathcal{F}_{2}\right)=Q_{1}\left(\underline{\sqrt{2 K_{\mathcal{S R}}}}, \sqrt{\mathcal{F}_{2}}\right)$ is strictly decreasing in $\mathcal{F}_{2}$. This implies that ${\overline{F_{\mathcal{P}_{\mathcal{S R}}}}}_{\boldsymbol{F}} \in[0,1]$ is positive increasing function of $\mu_{\mathcal{P}_{\mathcal{S R}}}$ because $\frac{\partial \mathcal{F}_{2}}{\partial \mu_{\mathcal{P}_{\mathcal{S R}}}}<0$. Finally using $\frac{\partial^{2} \mathcal{F}_{2}}{\partial \mu_{\mathcal{P}_{\mathcal{S R}}}{ }^{2}}=$ $\frac{4\left(K_{\mathcal{S R}}+1\right)\left(2^{2 R_{0}}-1\right) \sigma^{2}}{\mu_{\mathcal{P}_{\mathcal{S R}}}{ }^{3}} \geq 0, \log$-concavity of positive increasing $\overline{F_{\mathcal{P}_{r_{\mathcal{S R}}}}}$ in scaled transformation $\mathbb{E}\left[\gamma_{\mathcal{S R}}\right]=\frac{\mu_{\mathcal{P}_{\mathcal{S R}}}}{\sigma^{2}}$ is proved.

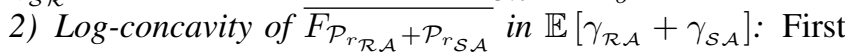
observe that MGF $\Phi_{\mathcal{P}_{r}}$ is strictly log-concave in $\mu_{\mathcal{P}}$, i.e., $\frac{\partial^{2} \log \left(\Phi_{\mathcal{P}_{r}}\right)}{\partial \mu_{\mathcal{P}}^{2}}=-\frac{\nu^{2}\left(2 K^{2}+3 K-\iota \mu_{\mathcal{P}} \nu+1\right)}{\left(K-\iota \mu_{\mathcal{P}} \nu+1\right)^{3}}<0$. As log-concavity is preserved under composition with affine function [35], log-concavity of $\Phi_{\mathcal{P}_{r_{1}}}\left(\nu, K_{1}, \mu_{\mathcal{P}_{1}}\right)$ in $\mu_{\mathcal{P}_{1}}$ also implies its log-concavity in $\mu_{\mathcal{P}_{1}}+\mu_{\mathcal{P}_{2}}$. Similarly log-concavity of $\Phi_{\mathcal{P}_{r_{2}}}\left(\nu, K_{2}, \mu_{\mathcal{P}_{2}}\right)$ in $\mu_{\mathcal{P}_{2}}$ implies its log-concavity in $\mu_{\mathcal{P}_{1}}+\mu_{\mathcal{P}_{2}}$. Also as log-concavity is preserved under positive product and integration [13], [35], $f_{\mathcal{P}_{r_{1}}+\mathcal{P}_{r_{2}}}=$ $\int_{-\infty}^{\infty} \frac{\mathrm{e}^{-\iota \nu x}}{2 \pi} \Phi_{\mathcal{P}_{r_{1}}}\left(\nu, K_{1}, \mu_{\mathcal{P}_{1}}\right) \Phi_{\mathcal{P}_{r_{2}}}\left(\nu, K_{2}, \mu_{\mathcal{P}_{2}}\right) d \nu$ is $\log -$ concave in $\mu_{\mathcal{P}_{1}}+\mu_{\mathcal{P}_{2}}$. Further $\overline{F_{\mathcal{P}_{r_{1}}+\mathcal{P}_{r_{2}}}}=\int^{\infty} f_{\mathcal{P}_{r_{1}}+\mathcal{P}_{r_{2}}} d x$ is also log-concave in $\mu_{\mathcal{P}_{1}}+\mu_{\mathcal{P}_{2}}$. From (20) ${ }^{x}$ we note that $F_{\mathcal{P}_{r_{1}}+\mathcal{P}_{r_{2}}}$ is decreasing function of both $\mu_{\mathcal{P}_{1}}$ and $\mu_{\mathcal{P}_{2}}$. This proves that $\overline{F_{\mathcal{P}_{r_{\mathcal{A} A}}+\mathcal{P}_{r_{\mathcal{S} A}}}}$ is increasing log-concave in $\mathbb{E}\left[\gamma_{\mathcal{R A}}\right], \mathbb{E}\left[\gamma_{\mathcal{S A}}\right]$, and $\mathbb{E}\left[\gamma_{\mathcal{R A}}+\gamma_{\mathcal{S} \mathcal{A}}\right]=\frac{\mu_{\mathcal{P}_{1}}+\mu_{\mathcal{P}_{2}}}{\sigma^{2}}$.

\section{APPENDIX C \\ PROOF OF THEOREM 2}

Before providing the proof for Theorem 2, we present two useful results in Lemma 5 and 6.

Lemma 5: The product $\overline{F_{\mathcal{P}_{r_{\mathcal{S R}}}}} \cdot \overline{F_{\mathcal{P}_{r_{\mathcal{R} A}}+\mathcal{P}_{r_{\mathcal{S A}}}}}$ is a positive log-concave function of $\alpha, \forall \mu_{0}>0$.

Proof: Let us consider $p_{\text {out }}^{\mathrm{IR}}=1-\left[\left(\mathcal{G}_{1} \circ \mathcal{H}_{1}\right)(\alpha)\right]$. $\left[\left(\mathcal{G}_{2} \circ \mathcal{H}_{2}\right)(\alpha)\right]$, where the composite functions $\left(\mathcal{G}_{1} \circ \mathcal{H}_{1}\right)(\alpha)=\overline{F_{\mathcal{P}_{\mathcal{S}_{\mathcal{R}}}}}$ and $\left(\mathcal{G}_{2} \circ \mathcal{H}_{2}\right)(\alpha)=\overline{F_{\mathcal{P}_{r_{\mathcal{R}}}+\mathcal{P}_{r_{\mathcal{S A}}}}}$. So, $\mathcal{G}_{1}=\overline{F_{\mathcal{P}_{\mathcal{S R}_{\mathcal{R}}}}}$ is a function of $\mathbb{E}\left[\gamma_{\mathcal{S R}}\right]$ and

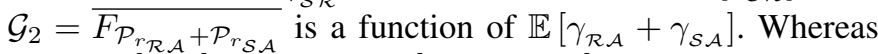
$\mathcal{H}_{1}=\mathbb{E}\left[\gamma_{\mathcal{S R}}\right]$ and $\mathcal{H}_{2}=\mathbb{E}\left[\gamma_{\mathcal{R A}}+\gamma_{\mathcal{S A}}\right]$ are functions of $\alpha$. Next we prove log-concavity of $\mathcal{G}_{1}\left(\mathcal{H}_{1}(\alpha)\right) \mathcal{G}_{2}\left(\mathcal{H}_{2}(\alpha)\right)$.

$$
\begin{gathered}
\frac{\partial^{2} \log _{2}\left[\mathcal{G}_{1}\left(\mathcal{H}_{1}(\alpha)\right) \mathcal{G}_{2}\left(\mathcal{H}_{2}(\alpha)\right)\right]}{\partial \alpha^{2}}=\underbrace{\left[\frac{\mathcal{G}_{1}^{\prime}\left(\mathcal{H}_{1}(\alpha)\right) \mathcal{H}_{1}^{\prime \prime}(\alpha)}{\mathcal{G}_{1}\left(\mathcal{H}_{1}(\alpha)\right)}+\frac{\mathcal{G}_{2}^{\prime}\left(\mathcal{H}_{2}(\alpha)\right) \mathcal{H}_{2}^{\prime \prime}(\alpha)}{\mathcal{G}_{2}\left(\mathcal{H}_{2}(\alpha)\right)}\right]}_{\text {first term }} \\
+\sum_{i=1}^{2} \underbrace{\left[\mathcal{H}_{i}^{\prime}(\alpha)\right]^{2}\left[\frac{\mathcal{G}_{i}\left(\mathcal{H}_{i}(\alpha)\right) \mathcal{G}_{i}^{\prime \prime}\left(\mathcal{H}_{i}(\alpha)\right)-\left[\mathcal{G}_{i}^{\prime}\left(\mathcal{H}_{i}(\alpha)\right)\right]^{2}}{\left[\mathcal{G}_{i}\left(\mathcal{H}_{i}(\alpha)\right)\right]^{2}}\right]} \quad \text { (C.1) }
\end{gathered}
$$

where $\mathcal{G}_{1}^{\prime}\left(\mathcal{H}_{1}(\alpha)\right)=\frac{\partial \overline{F_{\mathcal{P}_{r_{\mathcal{R}}}}}}{\partial \mathbb{E}\left[\gamma_{\mathcal{S R}}\right]}, \mathcal{G}_{1}^{\prime \prime}\left(\mathcal{H}_{1}(\alpha)\right)=\frac{\partial^{2} \overline{\bar{P}_{r_{\mathcal{S R}}}}}{\partial \mathbb{E}\left[\gamma_{\mathcal{S R}}\right]^{2}}$, $\mathcal{G}_{2}^{\prime}\left(\mathcal{H}_{2}(\alpha)\right)=\frac{\partial \overline{F_{\mathcal{P}_{\mathcal{R}_{\mathcal{A}}}+P_{r_{\mathcal{S}}}}}}{\partial \mathbb{E}\left[\gamma_{\mathcal{R}_{\mathcal{A}}}+\gamma_{\mathcal{S A}}\right]}, \mathcal{G}_{2}^{\prime \prime}\left(\mathcal{H}_{2}(\alpha)\right)=\frac{\partial^{2} \overline{F_{\mathcal{P}_{r_{\mathcal{A}}}+\mathcal{P}_{r_{\mathcal{S}}}}}}{\partial \mathbb{E}\left[\gamma_{\mathcal{R A} \mathcal{A}}+\gamma_{\mathcal{S A}}\right]^{2}}$, $\mathcal{H}_{1}^{\prime}(\alpha)=\frac{\partial \mathbb{E}\left[\gamma_{\mathcal{S R}}\right]}{\partial \alpha}, \mathcal{H}_{1}^{\prime \prime}(\alpha)=\frac{\partial^{2} \mathbb{E}\left[\gamma_{\mathcal{L}}\right]}{\partial \alpha^{2}}, \mathcal{H}_{2}^{\prime}(\alpha)=$ $\frac{\partial \mathbb{E}\left[\gamma_{\mathcal{R} \mathcal{A}}+\gamma_{\mathcal{S A}}\right]}{\partial \alpha}$, and $\mathcal{H}_{2}^{\prime \prime}(\alpha)=\frac{\partial^{2} \mathbb{E}\left[\gamma_{\mathcal{R} \mathcal{A}}+\gamma_{\mathcal{S A}}\right]}{\partial \alpha^{2}}$. Observe that the second and third terms in (C.1) are non-positive because $\frac{\partial^{2} \mathcal{G}_{1}}{\partial \mathcal{H}_{1}^{2}}=\frac{\mathcal{G}_{1}\left(\mathcal{H}_{1}(\alpha)\right) \mathcal{G}_{1}^{\prime \prime}\left(\mathcal{H}_{1}(\alpha)\right)-\left[\mathcal{G}_{1}^{\prime}\left(\mathcal{H}_{1}(\alpha)\right)\right]^{2}}{\left[\mathcal{G}_{1}\left(\mathcal{H}_{1}(\alpha)\right)\right]^{2}} \leq 0$ and $\frac{\partial^{2} \mathcal{G}_{2}}{\partial \mathcal{H}_{2}^{2}}=$ 
$\frac{\mathcal{G}_{2}\left(\mathcal{H}_{2}(\alpha)\right) \mathcal{G}_{2}^{\prime \prime}\left(\mathcal{H}_{2}(\alpha)\right)-\left[\mathcal{G}_{2}^{\prime}\left(\mathcal{H}_{2}(\alpha)\right)\right]^{2}}{\left[\mathcal{G}_{2}\left(\mathcal{H}_{2}(\alpha)\right)\right]^{2}} \leq 0$ due to log-concavity of $\mathcal{G}_{1}=\overline{F_{\mathcal{P}_{r_{\mathcal{R}}}}}$ in $\mathcal{H}_{1}=\mathbb{E}\left[\gamma_{\mathcal{S R}}\right]$ and $\mathcal{G}_{2}=\overline{F_{\mathcal{P}_{r_{\mathcal{A} A}}+\mathcal{P}_{r_{\mathcal{S A}}}}}$ in $\mathcal{H}_{2}=\mathbb{E}\left[\gamma_{\mathcal{R} \mathcal{A}}+\gamma_{\mathcal{S}_{\mathcal{A}}}\right]$ as proved in Theorem 1. Further from Corollary 2 and Lemma 4, we note that $\mathcal{H}_{1}=\mathbb{E}\left[\gamma_{\mathcal{S R}}\right]$ and $\mathcal{H}_{2}=\mathbb{E}\left[\gamma_{\mathcal{R A}}+\gamma_{\mathcal{S A}}\right]$ are concave functions of $\alpha$ for $\mu_{0}>0$. So in first term of (C.1), $\mathcal{H}_{1}^{\prime \prime}(\alpha) \leq 0$ and $\mathcal{H}_{2}^{\prime \prime}(\alpha) \leq 0$. Also from Theorem 1, $\mathcal{G}_{1}$ and $\mathcal{G}_{2}$ are positive increasing functions of $\mathcal{H}_{1}$ and $\mathcal{H}_{2}$ respectively. So in first term, $\mathcal{G}_{1}^{\prime}\left(\mathcal{H}_{1}(\alpha)\right)>0$ and $\mathcal{G}_{2}^{\prime}\left(\mathcal{H}_{2}(\alpha)\right)>0$, which proves $\frac{\partial^{2} \log _{2}\left[\mathcal{G}_{1}\left(\mathcal{H}_{1}(\alpha)\right) \mathcal{G}_{2}\left(\mathcal{H}_{2}(\alpha)\right)\right]}{\partial \alpha^{2}} \leq 0$, $\forall \mu_{0}>0$, because it is sum of three non-positive terms.

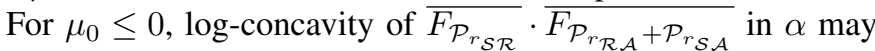
not hold, so following result is given.

Lemma 6: For $\mu_{0} \leq 0, \alpha_{g_{\mathrm{ER}}} \leq \alpha \leq \alpha_{g_{\mathrm{IR}}}, \overline{F_{\mathcal{P}_{r_{\mathcal{S R}}}}}$. $\overline{F_{\mathcal{P}_{r_{\mathcal{R} A}}+\mathcal{P}_{r_{\mathcal{S A}}}}}$ is a pseudoconcave function of $\alpha$.

Proof: To prove pseudoconcavity [34], we show that $\overline{F_{\mathcal{P}_{r_{\mathcal{R}}}}} \cdot \overline{F_{\mathcal{P}_{r_{\mathcal{R A}}}+\mathcal{P}_{r_{\mathcal{S A}}}}}$ is unimodal in $\alpha$.

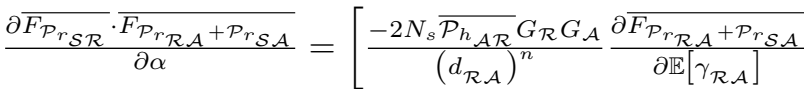

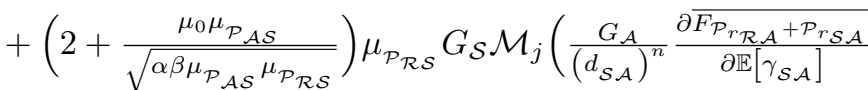

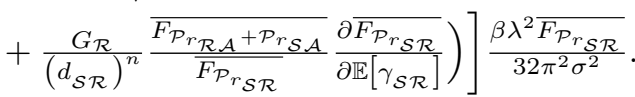

Using (C.2), the critical point of $\overline{F_{\mathcal{P}_{r_{\mathcal{S R}}}}} \cdot \overline{{\overline{\mathcal{P}_{r_{\mathcal{R}}}}}_{\mathcal{P}_{r_{\mathcal{S A}}}}}$ with respect to $\alpha$ is given by:

$$
\alpha_{c r i}^{\text {out }} \triangleq\left\{\alpha \mid \sqrt{\alpha}=\frac{\mu_{0}}{2} \sqrt{\frac{\mu_{\mathcal{P}_{\mathcal{A S}}} \mu_{\mathcal{P}_{\mathcal{R S}}}}{\beta}}\left[\mu_{\mathcal{P}_{\mathcal{R S}}}-\Psi\right]^{-1}\right\}
$$

with $\Psi \geq \mu_{\mathcal{P}_{\mathcal{R S}}}$ to ensure $\sqrt{\alpha} \geq 0$. Here $\Psi \triangleq \frac{\overline{\mathcal{P}_{h_{\mathcal{A}}}} N_{s} G_{\mathcal{A}} G_{\mathcal{R}}}{\left(d_{\mathcal{R A}}\right)^{n}}$

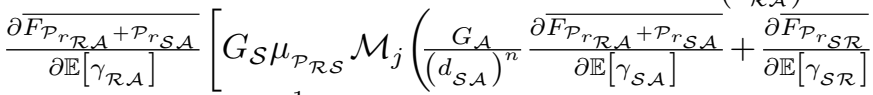

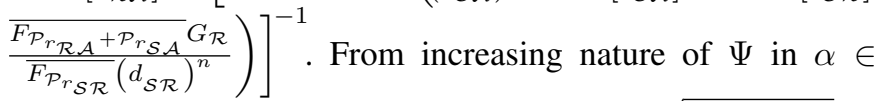
$\left[\alpha_{g_{\mathrm{ER}}}, \alpha_{g_{\mathrm{IR}}}\right] \forall \mu_{0} \leq 0$ (cf. Proposition 1), $\frac{\mu_{0} \sqrt{\mu_{\mathcal{P}_{\mathcal{A S}}} \mu_{\mathcal{P}_{\mathcal{R S}}}}}{2 \sqrt{\beta}\left(\mu_{\mathcal{P}_{\mathcal{R S}}}-\Psi\right)}$ is decreasing in $\alpha$. Further as $\sqrt{\alpha}$ is strictly increasing in $\alpha$, if $\exists \alpha_{c r i}^{\text {out }} \in\left[\alpha_{g_{\mathrm{ER}}}, \alpha_{g_{\mathrm{IR}}}\right]$, then it has to be unique because a strictly increasing and strictly decreasing function can cross each other only at a single point. This proves that $\overline{F_{\mathcal{P}_{r_{\mathcal{R}}}}} \cdot \overline{F_{\mathcal{P}_{r_{\mathcal{R} A}}+\mathcal{P}_{r_{\mathcal{S A}}}}}$ is positive pseudoconcave in $\alpha \in\left[\alpha_{g_{\mathrm{ER}}}, \alpha_{g_{\mathrm{IR}}}\right]$ for $\mu_{0} \leq 0$.

Proposition 1: For $\mu_{0} \leq 0, \Psi$, as defined below (C.3) in Lemma 6, is an increasing function of $\alpha \in\left[\alpha_{g_{\mathrm{ER}}}, \alpha_{g_{\mathrm{IR}}}\right]$ because

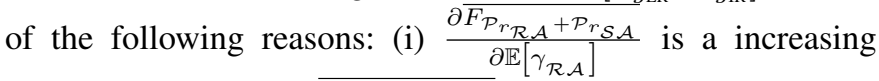
function of $\alpha$, (ii) $\frac{\partial \overline{F_{\mathcal{P}_{\mathcal{R} A}}+\mathcal{P}_{r_{\mathcal{S}}}}}{\partial \mathbb{E}\left[\gamma_{\mathcal{S A}}\right]}$ is a decreasing function of $\alpha$, (iii) $\overline{F_{\mathcal{P}_{r_{\mathcal{A}}}+\mathcal{P}_{r_{\mathcal{S A}}}}}$ is decreasing in $\alpha$, (iv) $\overline{F_{\mathcal{P}_{r_{\mathcal{S R}}}}}$ is a increasing function of $\alpha$, and (v) $\frac{\partial \overline{F_{\mathcal{P}_{\mathcal{S}} \mathcal{R}}}}{\partial \mathbb{E}\left[\gamma_{\mathcal{S R}}\right]}$ is decreasing in $\alpha$.

Proof: From Theorem 1, we note that $\overline{F_{\mathcal{P}_{r_{\mathcal{R}}}}}$ and $\overline{F_{\mathcal{P}_{r_{\mathcal{R} A}}}+\mathcal{P}_{r_{\mathcal{S}}}}$ are positive increasing log-concave functions of $\mathbb{E}\left[\gamma_{\mathcal{S R}}\right]$ and $\mathbb{E}\left[\gamma_{\mathcal{R A}}+\gamma_{\mathcal{S A}}\right]$ respectively. Also, $\frac{\partial \mathbb{E}\left[\gamma_{\mathcal{R} \mathcal{A}}\right]}{\partial \alpha}=$ $-\frac{G_{\mathcal{A}} \lambda^{2}}{(4 \pi)^{2} \sigma^{2}} \frac{\beta G_{\mathcal{R}} N_{s} \overline{\mathcal{P}_{h} \mathcal{A \mathcal { R }}}}{\left(d_{\mathcal{R} \mathcal{A}}\right)^{n}}$ shows that $\mathbb{E}\left[\gamma_{\mathcal{R}_{\mathcal{A}}}\right]$ is strictly decreasing in $\alpha$. Using these we observe that, though $\overline{F_{\mathcal{P}_{r_{\mathcal{R} A}}+\mathcal{P}_{r_{\mathcal{S}}}}}$ is positive increasing function of $\mathbb{E}\left[\gamma_{\mathcal{R A}}\right]$, its rate of increase is decreasing in $\mathbb{E}\left[\gamma_{\mathcal{R A}}\right]$, i.e., $\frac{\partial \overline{F_{\mathcal{P}_{\mathcal{R} A}}+\mathcal{P}_{\boldsymbol{P}_{\mathcal{A}} \mathcal{A}}}}{\partial \mathbb{E}\left[\gamma_{\mathcal{R}_{\mathcal{A}}}\right]}$ is decreasing

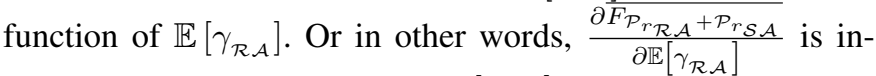
creasing function of $\alpha$ because $\mathbb{E}\left[\gamma_{\mathcal{R}_{A}}\right]$ is a strictly decreasing function of $\alpha$. This proves part (i). Similarly, as $\mathbb{E}\left[\gamma_{\mathcal{S A}}\right]$ is an increasing function of $\alpha, \frac{\partial \overline{F_{\mathcal{P}_{\mathcal{R} \mathcal{A}}}+\mathcal{P}_{\boldsymbol{r}_{\mathcal{A}}}}}{\partial \mathbb{E}\left[\gamma_{\mathcal{S} \mathcal{A}}\right]}$ is decreasing function of $\alpha$, which proves part (ii). So, numerator of $\Psi$ is increasing in $\alpha$ because it is product of positive constant $\frac{\overline{\mathcal{P}_{\mathcal{A}_{\mathcal{R}}}} N_{s} G_{\mathcal{A}} G_{\mathcal{R}}}{\left(d_{\mathcal{R}_{\mathcal{A}}}\right)^{n}}$

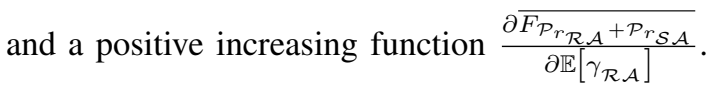

Next we show that denominator $G_{\mathcal{S}} \mu_{\mathcal{P}_{\mathcal{R}}} \mathcal{M}_{j}\left(\frac{G_{\mathcal{A}}}{\left(d_{\mathcal{S} \mathcal{A}}\right)^{n}}\right.$

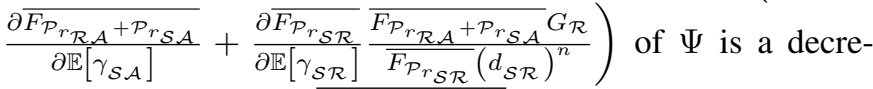
asing function of $\alpha . \overline{F_{\mathcal{P}_{R_{\mathcal{A}}}}+\mathcal{P}_{r_{\mathcal{S} A}}}$ is a decreasing function of $\alpha$ because $\mathbb{E}\left[\gamma_{\mathcal{R}_{\mathcal{A}}}+\gamma_{\mathcal{S A}}\right]$ is decreasing in $\alpha \forall \mu_{0} \leq 0$ and $\alpha \leq \alpha_{g_{\mathrm{IR}}}$ (cf. Lemma 4). This proves part (iii) of Proposition 1. From Theorem $1, \overline{F_{\mathcal{P}_{r_{\mathcal{S}}}}}$ is positive increasing function of $\mathbb{E}\left[\gamma_{\mathcal{S R}}\right]$, and hence increasing function of $\alpha$ also because $\mathbb{E}\left[\gamma_{\mathcal{S R}}\right]$ is increasing in $\alpha$. This proves part (iv). However its rate of increase is decreasing in $\mathbb{E}\left[\gamma_{\mathcal{S R}}\right]$. Or in other words, $\frac{\partial \overline{F_{\mathcal{P}_{\mathcal{S R}}}}}{\partial \mathbb{E}\left[\gamma_{\mathcal{S} \mathcal{R}}\right]}$ is decreasing function of $\alpha$ because $\mathbb{E}\left[\gamma_{\mathcal{S R}}\right]$ is a strictly increasing function of $\alpha$, which proves part (v). Observing results (ii)-(v), along with the fact that sum and product of positive decreasing functions is also decreasing, we note that the denominator of $\Psi$ is decreasing in $\alpha$. Finally, as the ratio of positive increasing and decreasing functions is an increasing function, $\Psi$ with its numerator and denominator respectively being positive increasing and decreasing functions of $\alpha$ proves that $\Psi$ itself is positive increasing in $\alpha$.

Now we use the above two results to prove Theorem 2. From Lemma 5 we note that $\overline{F_{\mathcal{P}_{r_{\mathcal{S R}}}}} \cdot \overline{F_{\mathcal{P}_{r_{\mathcal{R A}}}+\mathcal{P}_{r_{\mathcal{S A}}}}}$ is positive log-concave function of $\alpha \in[0,1], \forall \mu_{0}>0$. Further as a positive differentiable log-concave function defined over a convex set is pseudoconcave in nature [13, Lemma 5], $\overline{F_{\mathcal{P}_{r_{\mathcal{R}}}}} \cdot \overline{F_{\mathcal{P}_{r_{\mathcal{R}}}+\mathcal{P}_{r_{\mathcal{S A}}}}}$ is also pseudoconcave in $\alpha, \forall \mu_{0}>0$. From Lemma 6 , we observe that $\overline{F_{\mathcal{P}_{r_{\mathcal{R}}}}} \cdot \overline{F_{\mathcal{P}_{r_{\mathcal{A}}}}+\mathcal{P}_{r_{\mathcal{S A}}}}$ is pseudoconcave function of $\alpha \in\left[\alpha_{g_{\mathrm{ER}}}, \alpha_{g_{\mathrm{IR}}}\right]$. This proves that $p_{\text {out }}^{\mathrm{IR}}=1-\overline{F_{\mathcal{P}_{r_{\mathcal{S R}}}}} \cdot \overline{F_{\mathcal{P}_{r_{\mathcal{R}}}+\mathcal{P}_{r_{\mathcal{S A}}}}}$ is a positive pseudoconvex function of $\alpha \in\left\{\begin{array}{ll}{[0,1],} & \mu_{0}>0 \\ {\left[\alpha_{g_{\mathrm{ER}}}, \alpha_{g_{\mathrm{IR}}}\right],} & \mu_{0} \leq 0 .\end{array}\right.$ Thus, pseudoconvexity of objective function along with convexity of $C 2-C 3$ and [38, Theorem 4.3.8] shows that (P2) has unique globaloptimal $\alpha^{*} \in\left[\alpha_{g_{\mathrm{ER}}}, \alpha_{g_{\mathrm{IR}}}\right]$ that minimizes $p_{\text {out }}^{\mathrm{IR}}$.

\section{REFERENCES}

[1] D. Mishra, S. De, and D. Krishnaswamy, " $i^{2}$ ER: Integrated information and energy relaying protocol for RF powered communication network," in Proc. IEEE ICC, Paris, May 2017, pp. 1317-1322.

[2] X. Lu et al., "Wireless networks with RF energy harvesting: A contemporary survey," IEEE Commun. Surveys Tuts., vol. 17, no. 2, pp. 757-789, Second quarter 2015.

[3] H. Tabassum et al., "Wireless-powered cellular networks: Key challenges and solution techniques," IEEE Commun. Mag., vol. 53, no. 6, pp. 63-71, June 2015.

[4] I. Krikidis et al., "Simultaneous wireless information and power transfer in modern communication systems," IEEE Commun. Mag., vol. 52, no. 11, pp. 104-110, Nov. 2014. 
[5] D. Mishra and S. De, "Optimal relay placement in two-hop RF energy transfer," IEEE Trans. Commun., vol. 63, no. 5, pp. 1635-1647, May 2015.

[6] H. Ju and R. Zhang, "User cooperation in wireless powered communication networks," in Proc. IEEE GLOBECOM, Austin, Dec. 2014, pp. 1430-1435.

[7] H. Chen et al., "Harvest-then-cooperate: Wireless-powered cooperative communications," IEEE Trans. Signal Process., vol. 63, no. 7, pp. 17001711, Apr. 2015.

[8] Y. Gu et al., "An adaptive transmission protocol for wireless-powered cooperative communications," in Proc. IEEE ICC, London, June 2015, pp. 4223-4228.

[9] H. Ding et al., "Role selection for energy harvesting cooperative systems," in Proc. IEEE GLOBECOM, San Diego, Dec. 2015, pp. 1-6.

[10] Z. Ding et al., "Power allocation strategies in energy harvesting wireless cooperative networks," IEEE Trans. Wireless Commun., vol. 13, no. 2, pp. 846-860, Feb. 2014.

[11] B. Gurakan et al., "Optimal energy and data routing in networks with energy cooperation," IEEE Trans. Wireless Commun., vol. 15, no. 2, pp. 857-870, Feb. 2016.

[12] X. Huang and N. Ansari, "Energy sharing within EH-enabled wireless communication networks," IEEE Wireless Commun., vol. 22, no. 3, pp 144-149, June 2015.

[13] D. Mishra et al., "Joint optimization schemes for cooperative wireless information and power transfer over rician channels," IEEE Trans. Commun., vol. 64, no. 2, pp. 554-571, Feb 2016.

[14] D. Michalopoulos et al., "Relay selection for simultaneous information transmission and wireless energy transfer: A tradeoff perspective," IEEE J. Sel. Areas Commun., vol. 33, no. 8, pp. 1578-1594, Aug. 2015.

[15] S. Lohani et al., "On multiuser resource allocation in relay-based wireless-powered uplink cellular networks," IEEE Trans. Wireless Commun., vol. 15, no. 3, pp. 1851-1865, Mar. 2016.

[16] I. Krikidis et al., "RF energy transfer for cooperative networks: Data relaying or energy harvesting?" IEEE Commun. Lett., vol. 16, no. 11, pp. 1772-1775, Nov. 2012.

[17] Y. Gu et al., "Distributed multi-relay selection in wireless-powered cooperative networks with energy accumulation," in Proc. IEEE ICC, Kuala Lumpur, May 2016, pp. 1-6.

[18] K. H. Liu, "Performance analysis of relay selection for cooperative relays based on wireless power transfer with finite energy storage," IEEE Trans. Veh. Technol., vol. 65, no. 7, pp. 5110-5121, July 2016.

[19] L. Tang et al., "Wireless information and energy transfer in fading relay channels," IEEE J. Sel. Areas Commun., vol. 34, no. 12, pp. 3632-3645, Dec. 2016.

[20] M. Mohammadi et al., "Throughput analysis and optimization of wireless-powered multiple antenna full-duplex relay systems," IEEE Trans. Commun., vol. 64, no. 4, pp. 1769-1785, Apr. 2016.

[21] H. Ju and R. Zhang, "Throughput maximization in wireless powered communication networks," IEEE Trans. Wireless Commun., vol. 13, no. 1, pp. 418-428, Jan. 2014.

[22] P. Nintanavongsa et al., "Design optimization and implementation for RF energy harvesting circuits," IEEE J. Emerg. Sel. Topics Circuits Syst., vol. 2, no. 1, pp. 24-33, Mar. 2012.

[23] P.-H. Hsieh et al., "An RF energy harvester with 44.1\% PCE at input available power of -12 dbm," IEEE Trans. Circuits Syst. I, Reg. Papers, vol. 62, no. 6, pp. 1528-1537, June 2015.

[24] Powercast. Accessed Aug. 10, 2015. [Online]. Available: http://www.powercastco.com.

[25] AVX BestCap®. Accessed Nov. 15, 2015. [Online]. Available: http://www.avx.com/products/supercapacitors/bestcapreg-bz-series/

[26] D. Mishra and S. De, "Effects of practical rechargeability constraints on perpetual RF harvesting sensor network operation," IEEE Access, vol. 4, pp. 750-765, Mar. 2016.

[27] D. Jenn, "Transmission equation for multiple cooperative transmitters and collective beamforming," IEEE Antennas Wireless Propag. Lett., vol. 7, pp. 606-608, Aug. 2008.

[28] I. S. Gradshteyn and I. M. Ryzhik, Table of integrals, series, and products, 7th ed. Academic Press, Amsterdam, 2007.

[29] M. K. Simon and M.-S. Alouini, Digital communication over fading channels, 2nd ed. New Jersey: John Wiley \& Sons, 2005, vol. 95

[30] P. Billingsley, Probability and measure, 3rd ed. New York: John Wiley \& Sons, 2008.

[31] E. W. Weisstein, "Lambert W-Function," From MathWorld - A Wolfram Web Resource, accessed Oct. 25, 2015. [Online]. Available: http://mathworld.wolfram.com/LambertW-Function.html
[32] A. Castano-Martinez and F. Lopez-Blazquez, "Distribution of a sum of weighted noncentral chi-square variables," TEST, vol. 14, no. 2 , pp. 397-415, Dec. 2005.

[33] A. Cambini and L. Martein, Generalized convexity and optimization: Theory and applications. Springer, 2008, vol. 616.

[34] M. Avriel et al., Generalized concavity. Philadelphia, PA, USA: SIAM, vol. 63, 2010.

[35] S. Boyd and L. Vandenberghe, Convex optimization. Cambridge university press, 2004.

[36] Y. Yu, "On Log-concavity of the Generalized Marcum Q Function," ArXiv e-prints, vol. arXiv:1105.5762, May 2011. [Online]. Available: http://adsabs.harvard.edu/abs/2011arXiv1105.5762Y

[37] Y. Sun et al., "On the monotonicity, log-concavity, and tight bounds of the generalized Marcum and Nuttall Q -functions," IEEE Trans. Inf. Theory, vol. 56, no. 3, pp. 1166-1186, Mar. 2010.

[38] M. S. Bazaraa et al., Nonlinear Programming: Theory and Applications, 3rd ed. New Jersey: John Wiley \& Sons, 2006.

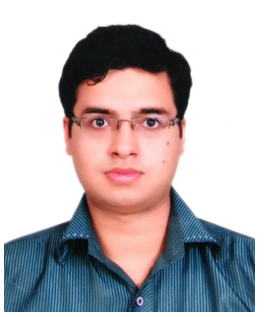

Deepak Mishra (S'13) received his B.Tech degree in Electronics and Communication Engineering from Guru Gobind Singh Indraprastha University, Delhi, India, in 2012 . He is currently pursuing the Ph.D. degree from the Department of Electrical Engineering, IIT Delhi, India. He is a recipient of 2016 IBM Ph.D. Fellowship award and 2017 RamanCharpak Fellowship award. His research interests include energy harvesting cooperative communication networks and energy optimization schemes for uninterrupted operation of mobile ad hoc networks.

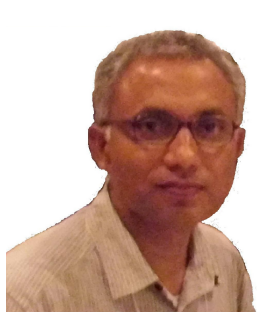

Swades De (S'02-M'04-SM'14) received the $\mathrm{PhD}$ degree from the State University of New York at Buffalo, NY, in 2004. From 1993 to 1997 and in 1999, he worked in different communication companies in India as a research and development engineer. In 2004 he worked at ISTI-CNR, in Pisa, Italy, as an ERCIM fellow. From 2004 to 2006 he was with the New Jersey Institute of Technology, as a TenureTrack Assistant Professor of ECE. He joined the Department of Electrical Engineering at IIT Delhi in 2007, where he is currently a Professor. Dr. De is a Senior Member of IEEE and a Fellow of the INAE. Currently he serves as a Senior Editor of IEEE Communications Letters and Associate Editor respectively of Springer Photonic Network Communications journal and IETE Technical Review journal.

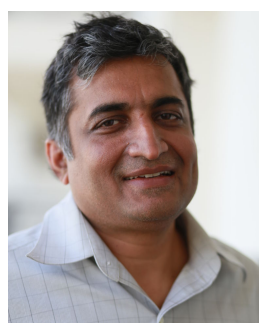

Dr. Dilip Krishnaswamy is currently working as a Senior Scientist at IBM Research in Bangalore, India. He received the $\mathrm{PhD}$ degree in electrical engineering from the University of Illinois at UrbanaChampaign. He has worked as a Platform Architect at Intel Corporation in Folsom, California, and as a Senior Staff Researcher in the Office of the Chief Scientist at the Qualcomm Research Center in San Diego. He has taught at the University of California, Davis, and has served on the Industrial Board of Advisors for the ECE dept at UC Davis. He served as the Associate Editor-in-Chief of the IEEE Wireless Communications magazine from 2009-2014. He in an inventor on 55 granted US patents, and has published $60+$ research papers with 3 best paper awards. 